\title{
Palladium-Catalyzed Isomerization-Coupling Reactions of Allyl Chloride with Amines to Generate Functionalized Phosphorus Derivatives
}

\author{
Jing-Hong Wen ${ }^{1,+}$, Qiang $\mathrm{Li}^{1,+}{ }^{+}$, Shao-Zhen Nie ${ }^{1}$, Jing-Jing $\mathrm{Ye}^{1}$, Qing $\mathrm{Xu}^{2}$ and \\ Chang-Qiu Zhao ${ }^{1, *}$ \\ 1 College of Chemistry and Chemical Engineering, Liaocheng University, Liaocheng 252059, China; \\ wenjinghongJ@163.com (J.-H.W.); liqiang9@lcu.edu.cn (Q.L.); nieshaozhen@163.com (S.-Z.N.); \\ jing-jingye@outlook.com (J.-J.Y.) \\ 2 College of Chemistry and Materials Engineering, Wenzhou University, Wenzhou 325035, China; \\ qing-xu@wzu.edu.cn \\ * Correspondence: literabc@hotmail.com; Tel.: +86-635-823-9659; Fax: +86-635-823-9121 \\ + These two authors contributed equally to this work.
}

Received: 17 April 2018; Accepted: 27 April 2018; Published: 5 May 2018

\begin{abstract}
A Pd-catalyzed isomerization-coupling reaction of $P$-containing allyl chloride with amine afforded imine or enamine that was converted to various functionalized phosphorus derivatives via hydrolysis, reduction, or Stork reactions. The reaction was confirmed to proceed via an isomerization of a starting material and a coupling of the resulting vinyl chloride with amine.
\end{abstract}

Keywords: isomerization-coupling; allyl chloride; amines; phosphorus derivatives; Stork reactions

\section{Introduction}

The functionalized phosphorus derivatives have attracted extensive attention in recent years because of their wide pharmacological and biological applications. For example, $\alpha$ - and $\beta$-aminophosphonates are used as antibiotics, herbicides, antifungal agents, and enzyme inhibitors [1-9]. $\gamma$-Aminophosphonates and their analogues have been reported to show activity as receptor agonists and antagonists [10]. 2-Amino-3-phosphonopropanoic acid and 2-amino-4-phosphonobutyric acid are known to show potent selective antagonist activities against glutamate receptors, to show antiviral activity, and as herbicides widely used in genetically modified and glufosinate-tolerant crops [11-14]. The compounds are also widely applied in asymmetric synthesis as important auxiliaries, P-N or P-O ligands, or their precursors, via the formation of chelating five- or six-membered cycles with metallic ions [15-19].

Although the functional phosphorus derivatives are of high importance, the procedures to generate them always involve in multi-step reactions or special precursors, especially for the $\gamma$-functionalized phosphonates. For example, Palacios reported the introduction of the $\gamma$-amino via an aza-Michael addition to a $\beta$-unsaturated imine [20]. Cytlak reported the preparation of trifluoromethyl $\gamma$-aminophosphonates by nucleophilic aziridine ring opening [21]. The compounds could be prepared starting from an $\alpha$-amino aldehyde via a Horner-Wadsworth-Emmons (HWE) reaction and subsequent conversions [10,22]. Straightforward and easy-to-handle methods to obtain the compounds still remain a challenge to chemists.

The Ullmann-type reactions can be effectively applied in the construction of C-N bonds via the coupling of aromatic halides with amines [23-25]. More recently, the Ullmann-Goldberg reactions between vinyl halides and amides have been developed [26-38], which can be effectively used in the preparation of $\mathrm{N}$-containing heterocycles. Normally, the coupling of vinyl halides to an amine is 
expected to form enamines or imines, which are versatile intermediates for the generation of various functionalized substances. Additionally, the similar coupling between allyl halide and an amine is hoped to afford an allylic amine, which could also be converted to enamine or imine after isomerization. However, to the best of our knowledge, the generation of enamine or imine via a coupling and/or an isomerization reaction of allyl halide has rarely been reported (Chart 1) [31,39].

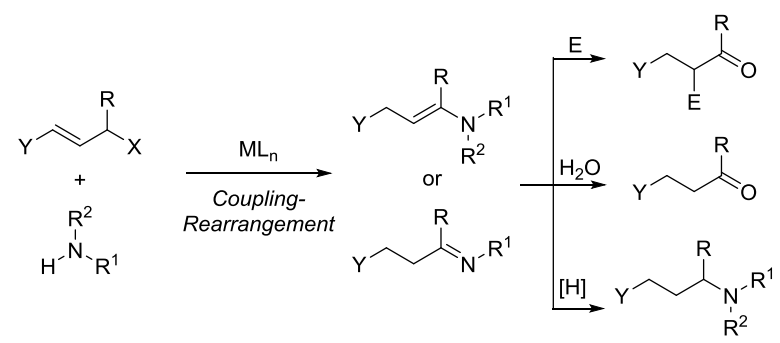

Chart 1. Supposed coupling-isomerization reactions of allyl halide with amines and the applications.

By means of the strategy of Chart 1 , the enamine or imine of ketones, which are normally more difficult to prepare that those of aldehydes [40], will be generated conveniently via the reaction of non-terminal allyl halide. Herein, we reported a palladium-catalyzed reaction of $\gamma$-chloro vinyl phosphorus derivative $\mathbf{1}$ with an amine, which afforded $\gamma$-imino or $\gamma$-enamino phosphorus derivatives via a coupling-isomerization reaction. The products could be conveniently converted to various $\gamma$-functionalized phosphorus derivatives, such as $\gamma$-keto, $\gamma$-hydroxyl, and $\gamma$-aminophosphonates and their analogues.

\section{Results and Discussion}

$\gamma$-Chlorovinylphosphinate 1a was obtained from the addition of $H$-phosphinate to cinnamic aldehyde and the subsequent chlorination of $\alpha$-hydroxy allyl phosphinate 2a [41]. As seen in Scheme 1 , 1a to $\mathbf{1 g}$ were prepared in excellent yields (NMR spectrum as seen in SM, Part 3). The copper-catalyzed coupling of $\mathbf{1}$ with amine 3a was firstly examined with reference to the reported synthesis of $\mathrm{N}$-heterocycles via the reaction of vinyl halide with an amine [26]. When 1d was heated with the $n$-butyl amine 3a in the presence of cuprous iodide and a ligand (1,10-phenanthroline or glycine), the coupling product was not detected. Instead, the isolation of $\alpha$-chloroallylphosphinate $4 \mathbf{d}^{\prime}$ was obtained as seen the dd peaks at 5.38 and $7.10 \mathrm{ppm}$ on the ${ }^{1} \mathrm{H}-\mathrm{NMR}$ spectrum (Scheme 2, NMR spectrum as seen in SM, Part 3) [42].

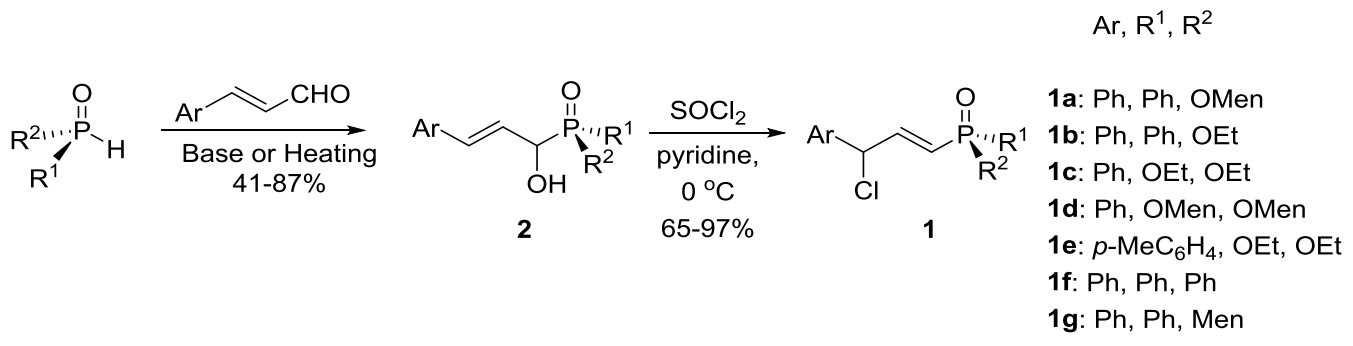

Scheme 1. The preparation of $1 \mathrm{a}$ to $1 \mathrm{~g}$ from H-P species and cinnamic aldehydes.

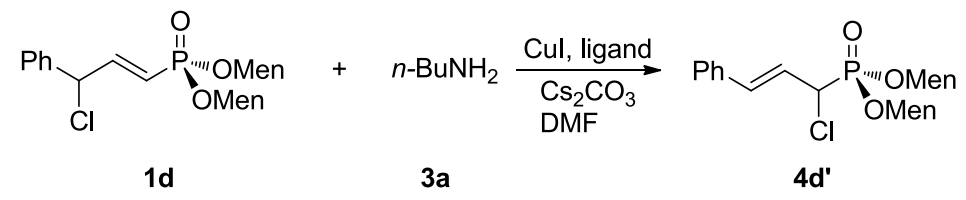

Scheme 2. The cuprous-iodide-catalyzed reaction of $1 \mathbf{d}$ with an amine. 
The palladium catalyst was thereafter examined for the coupling reaction of $\mathbf{1}$ with an amine. When 1a was heated with 3a in the presence of palladium acetate and triphenyl phosphine (TPP), two products, vinyl chloride $4 \mathbf{a}$ and $\gamma$-keto phosphinate $\mathbf{5 a}$, were isolated (Table 1). The formation of $4 \mathbf{a}$ was proved by a quartet peak of a vinyl proton at $6.20 \mathrm{ppm}$ on the ${ }^{1} \mathrm{H}-\mathrm{NMR}$ spectrum [43]. The structure of $\mathbf{5 a}$ was confirmed by X-ray diffraction results (Figure 1, as seen in SM, Part 1). Directive allylation of amine 3a with 1a was not detected.

Table 1. The optimization of the reaction conditions of 1a with 3a.

\begin{tabular}{|c|c|c|c|c|c|c|c|c|}
\hline \multirow[b]{2}{*}{ Entry } & $\overbrace{\mathrm{Cl}}^{\mathrm{Ph}}$ & \multicolumn{2}{|c|}{ 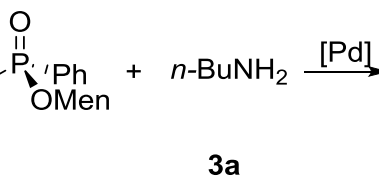 } & $-\stackrel{\mathrm{H}_{3} \mathrm{O}^{+}}{\longrightarrow} \mathrm{Ph}$ & \multicolumn{2}{|c|}{$\begin{array}{l}\mathrm{O}_{\text {OMen }}^{\mathrm{II}} \\
\mathbf{4 a}\end{array}$} & $\begin{array}{l}\mathrm{O} \\
\mathrm{Ia}\end{array}$ & \multirow[b]{2}{*}{$5 a: 4 a^{a}$} \\
\hline & $3 a$ (eq.) & Catalyst (\%) & $\mathrm{PPh}_{3}(\%)$ & Solvent & Temp $\left({ }^{\circ} \mathrm{C}\right)$ & Time (h) & Yield of 5a & \\
\hline 3 & 1.2 & $\mathrm{Pd}(\mathrm{OAc})_{2}, 5$ & 20 & toluene & 100 & 11 & 46 & $49: 51$ \\
\hline 4 & 2.2 & $\mathrm{Pd}(\mathrm{OAc})_{2}, 5$ & 20 & toluene & 100 & 13 & 67 & $72: 28$ \\
\hline 5 & 2.2 & $\mathrm{Pd}(\mathrm{OAc})_{2}, 5$ & 10 & toluene & 100 & 13 & 27 & $30: 70$ \\
\hline 6 & 2.2 & $\mathrm{Pd}(\mathrm{OAc})_{2}, 5$ & 20 & toluene & 120 & 24 & 99 & $>99: 1$ \\
\hline 7 & 2.2 & $\mathrm{PdCl}_{2}\left(\mathrm{PPh}_{3}\right)_{2}, 5$ & 20 & toluene & 120 & 24 & 93 & $>99: 1$ \\
\hline 12 & 2.2 & no & 20 & No & 120 & 24 & $-{ }^{c}$ & $<1: 99$ \\
\hline
\end{tabular}

${ }^{a}$ Carrying out the reaction in the typical procedure: a mixture of $1 \mathbf{a}(50 \mathrm{mg}, 0.116 \mathrm{mmol}), 3 \mathbf{a}(25 \mu \mathrm{L}, 0.255 \mathrm{mmol})$, palladium acetate $(1.3 \mathrm{mg}, 0.0058 \mathrm{mmol})$, and triphenyl phosphine $(6.1 \mathrm{mg}, 0.0232 \mathrm{mmol})$ in toluene $(0.5 \mathrm{~mL})$ was heated at $120{ }^{\circ} \mathrm{C}$. The yield and ratio were estimated by the ${ }^{31} \mathrm{P}\left\{{ }^{1} \mathrm{H}\right\}$ NMR spectrum. The peaks at 42.2 and $37.3 \mathrm{ppm}$ were assigned as $\mathbf{5 a}$ and $\mathbf{4 a}$, respectively; ${ }^{\mathrm{b}}$ The peaks at 25.1 and $21.7 \mathrm{ppm}$ (assigned as H-P species) and $40.4 \mathrm{ppm}$ (unknown) were detected; ${ }^{c}$ Only the peak at $37.3 \mathrm{ppm}(\mathbf{4 a})$ was detected by the ${ }^{31} \mathrm{P}-\mathrm{NMR}$ spectrum.

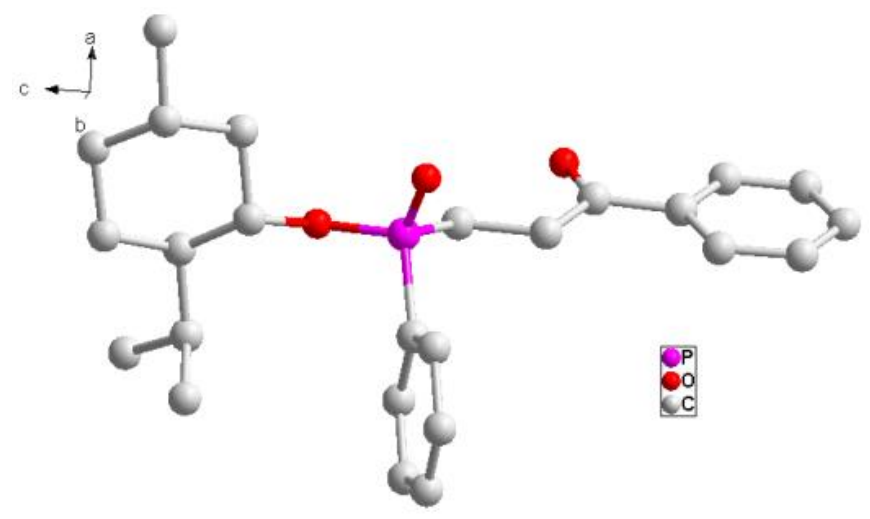

Figure 1. X-ray crystal structure of 5a.

When 1a was heated with one equivalent of $3 \mathbf{a}$ at $100{ }^{\circ} \mathrm{C}$ for $13 \mathrm{~h}$, $\mathbf{5 a}$ was formed with a $46 \%$ yield (entry 3). When two molar of $3 a$ was used, the yield of 5 a was increased to $67 \%$ (entry 4 ). Carrying out the reaction at $120^{\circ} \mathrm{C}$ gave nearly an entire formation of $\mathbf{5 a}(>99 \%$, entry 6$)$. A decreasing amount of TPP resulted in a reduced yield of $\mathbf{5 a}$. The absence of either TPP or palladium led to an entire conversion of $\mathbf{1 a}$ to $\mathbf{4} \mathbf{a}$ with no detection of $\mathbf{5 a}$ (entries 11-12). The results indicated that the palladium and TPP were essential to the formation of $\mathbf{5 a}$. Palladium chloride gave slightly poorer results than an acetate analog (entry 7). When the reactions were carried out in a polar solvent, such as DMA, DME, or NMP, an unsatisfactory yield of $5 \mathbf{a}$ was obtained (entries 8-10).

In a separate experiment, when 1a (together with its stereoisomer 1a' deriving from chiral $\gamma$-carbon) was heated with $3 \mathbf{a}$ at $120{ }^{\circ} \mathrm{C}$ for $24 \mathrm{~h}$, an entire isomerization of $1 \mathrm{a}$ to $4 \mathbf{a}$ was observed. 
When palladium acetate and TPP were added, $4 \mathbf{a}$ was consumed partly after $12 \mathrm{~h}$ and entirely within $24 \mathrm{~h}$ (Figure 2). $\gamma$-Keto phosphinate (5a) was obtained after quenching with aqueous ammonium chloride. The reaction of isolated $4 \mathbf{a}$ with $3 \mathbf{a}$ gave the same results.

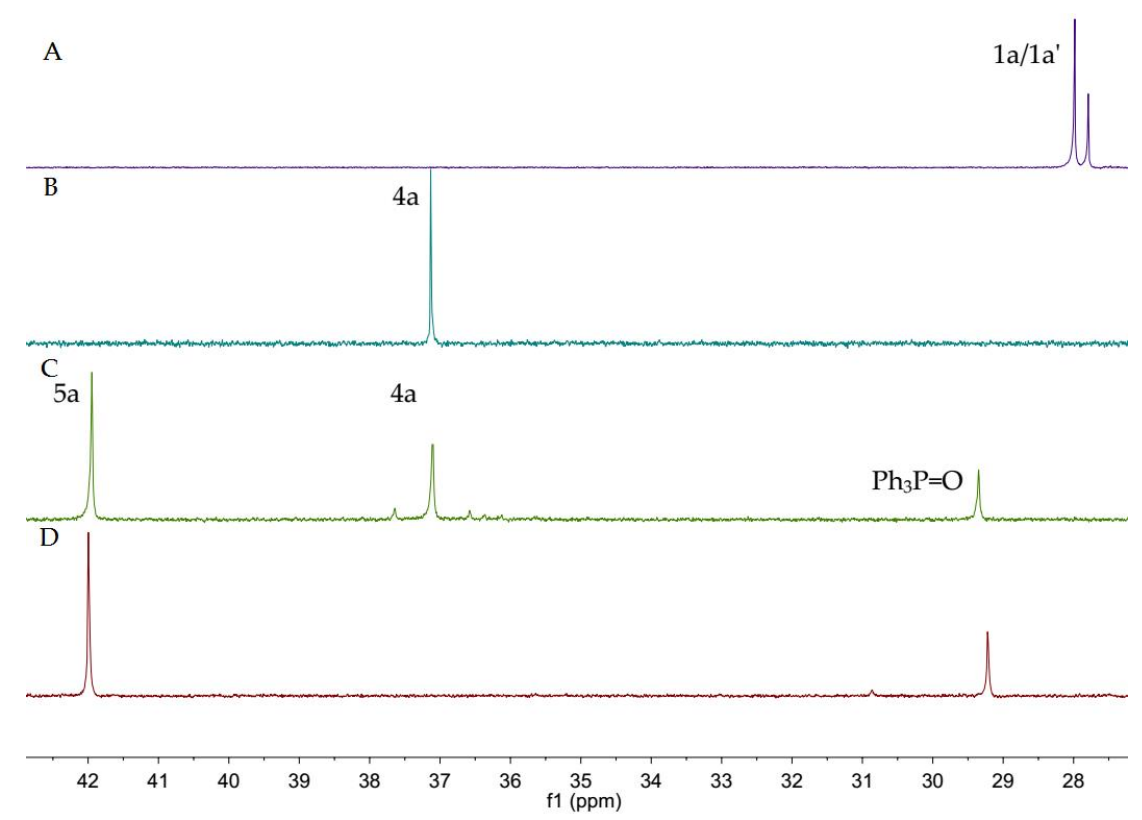

Figure 2. The variation of the NMR spectrum for the conversion of 1a to 5a. (A) Pure 1a/1a'; (B) Heating of 1a/1a' and $\mathbf{3 a}$ at $120^{\circ} \mathrm{C}$ for $24 \mathrm{~h}$; (C) Addition of $\mathrm{Pd}(\mathrm{OAc})_{2}$ and TPP with heating at $120^{\circ} \mathrm{C}$ for $12 \mathrm{~h}$; (D) The mixture was heated for $24 \mathrm{~h}$.

The palladium-catalyzed cross-coupling between $\mathbf{3 a}$ and $1 \mathbf{1 a} / \mathbf{4 a}$ was supposed to form the corresponding enamine $\mathbf{6 a}$ and then the imine $7 \mathbf{a}$ that was converted to $5 \mathbf{a}$ after removing the amine moieties via hydrolysis. As seen in Scheme 3, 1 was converted to 4 when heated in the presence of an amine. Vinyl chloride 4 combined with palladium via oxidative addition to form the vinyl palladium complex 8 that was converted to 9 via the coordination of the amine. The dehydrochlorination of 9 afforded 10. During the reductive elimination of 10, a $\gamma$-coupling product was formed. As for $\mathbf{1 a} / \mathbf{4 a}$, the amino only linked to $\gamma$-carbon to generate enamine 6 or imine 7. No formation of an $\alpha$-coupling product indicated that the reaction proceeded via vinyl palladium species as intermediates (as referred to in Scheme 5, vide infra).

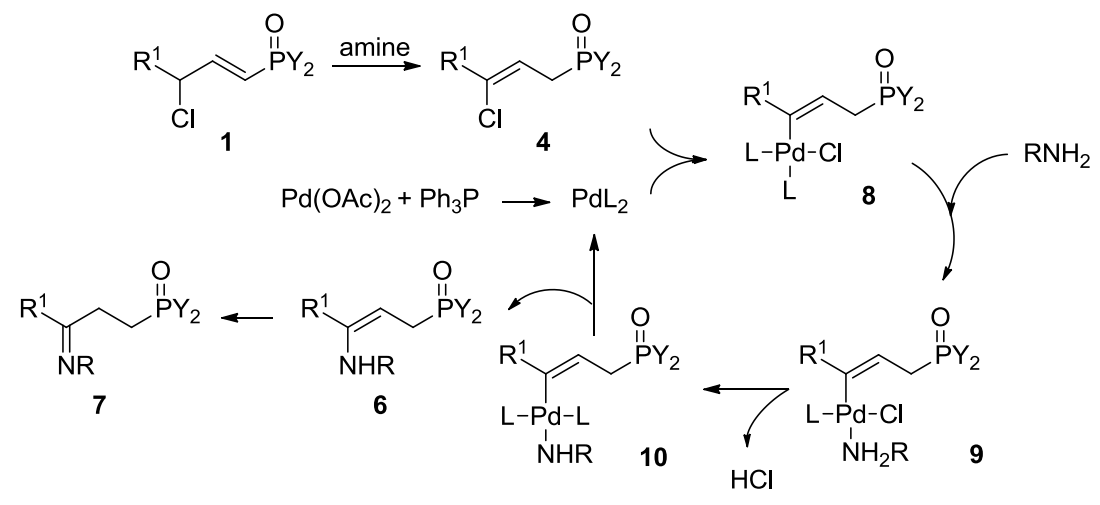

Scheme 3. The proposed mechanism for the isomerization-coupling reaction of the allyl chloride $\mathbf{1}$ with an amine. 
The $\gamma$-Keto phosphorus derivative $\mathbf{5}$ was formed from the hydrolysis of $\mathbf{6}$ or $\mathbf{7}$. Although the $\gamma$-imino derivative 7 was not obtained, it could be observed by means of the NMR spectrum. As seen in Figure 3, the coupling of $\mathbf{1 f}$ with a benzyl amine afforded $\mathbf{7 f}$, whose cis/trans isomers gave four proton signals $\left(\mathrm{H}_{\mathrm{A}}\right.$ and $\left.\mathrm{H}_{\mathrm{B}}\right)$ around 3.07 to $2.70 \mathrm{ppm}$. Partial hydrolysis of $\mathbf{7 f}$ resulted in the formation of $5 \mathbf{f}$ that gave two proton singles of $\mathrm{H}_{\mathrm{C}}$ and $\mathrm{H}_{\mathrm{D}}$ at 3.36 and $2.70 \mathrm{ppm}$, respectively. On the ${ }^{31} \mathrm{P}-\mathrm{NMR}$ spectrum, cis/trans-7f gave two peaks at 33.0 and $31.2 \mathrm{ppm}$. During the treatment with aqueous ammonium chloride, an obviously increasing peak of $5 \mathrm{f}$ at $32.7 \mathrm{ppm}$ was observed accompanied by decreasing peaks of $\mathbf{7 f}$ (NMR spectrum, as seen in SM, Part 2).
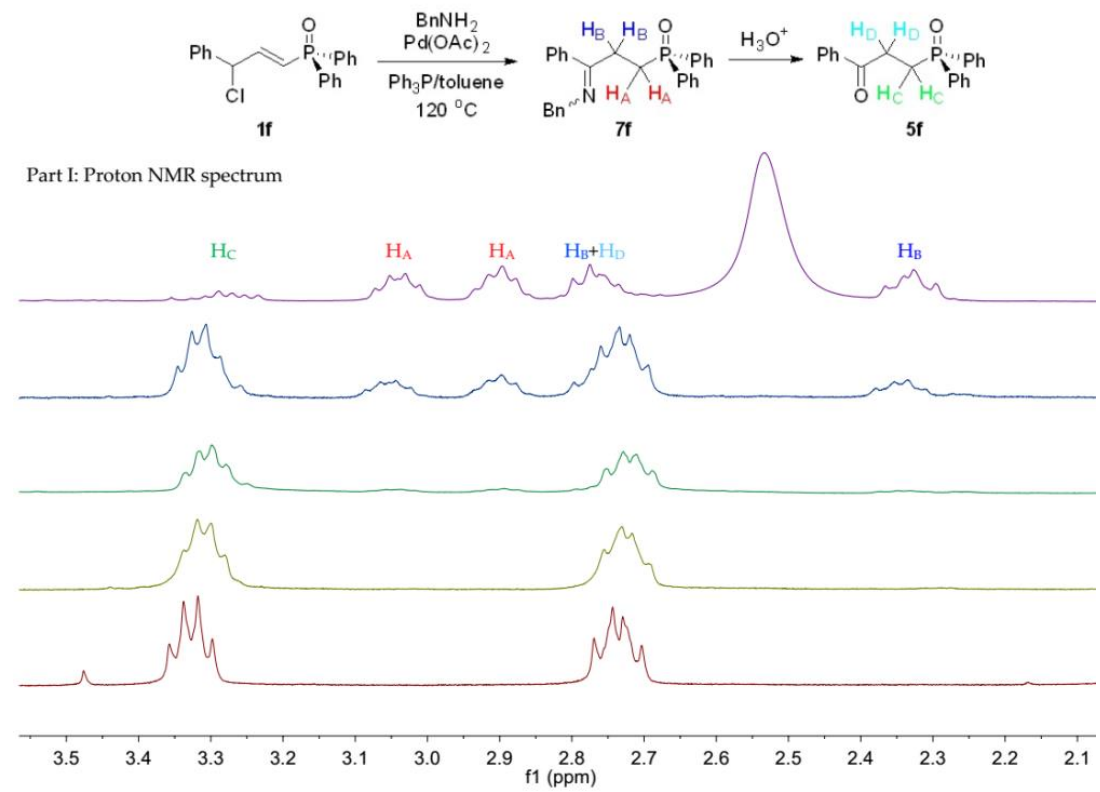

Part II: ${ }^{31 P}$ NMR spectrum

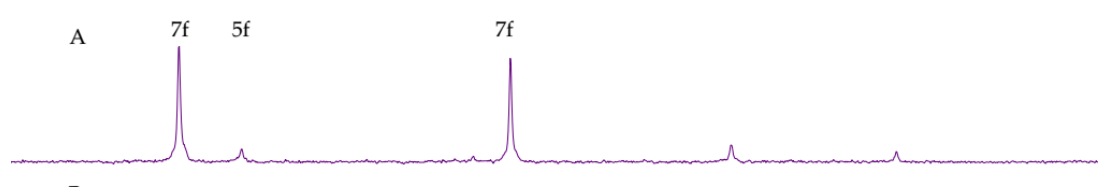

B

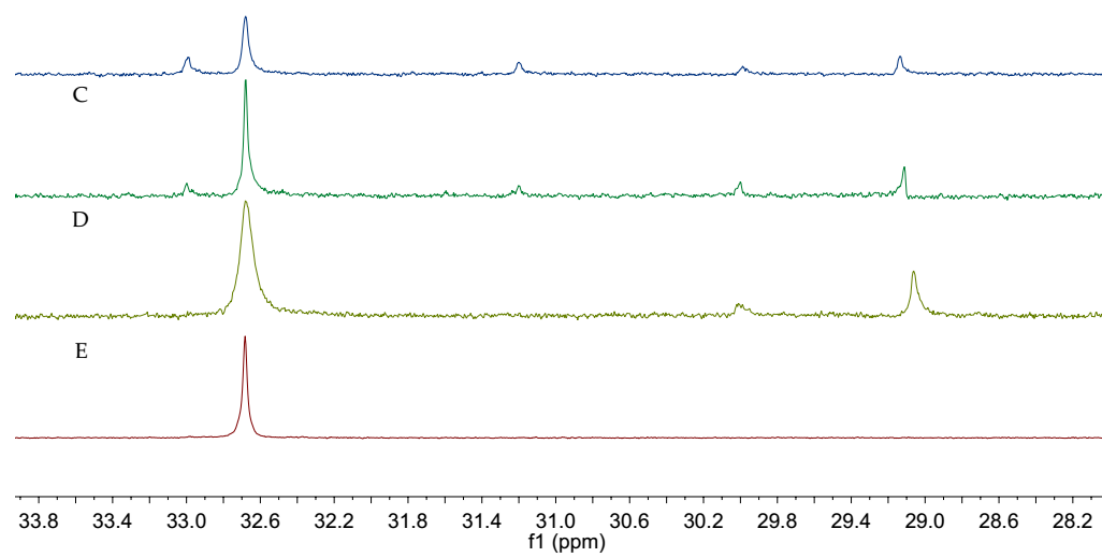

Figure 3. The variation in NMR spectrum for the reaction of $\mathbf{1 f}$ with a benzyl amine. Part I. Proton NMR spectrum; Part II. ${ }^{31}$ P-NMR spectrum. (A) The crude product was analyzed with an NMR spectrum; (B) The solution of crude product was washed with aqueous ammonium chloride; (C) Further washing of the solution with aqueous ammonium chloride; (D) The solution of crude product was washed with diluted hydrochloric acid; (E) Isolated $5 \mathbf{f}$. 
The isomerization-coupling reaction could be applied for the preparation of various phosphorus derivatives. Firstly, the $\gamma$-keto derivatives 5 were obtained via the hydrolysis of $\mathbf{6}$ or $\mathbf{7}$ (Table 2) [44,45]. The primary amines that contained primary, secondary, or tertiary alkyl groups afforded the same $5 \mathbf{a}$. The coupling of a secondary amine, such as piperidine, also afforded $5 \mathbf{a}$ via the intermediate 6 . Other $\gamma$-keto phosphorus derivatives, such as ethyl phenylphosphinate $\mathbf{5 b}$, diethyl phosphonate $\mathbf{5 c}$, dimenthyl phosphonate $\mathbf{5 d}$, and diphenyl phosphine oxides $\mathbf{5 f}$, were similarly obtained in moderate to excellent yields (NMR spectrum as seen in SM, Part 3).

Table 2. Preparation of the $\gamma$-keto phosphorus derivatives 5.

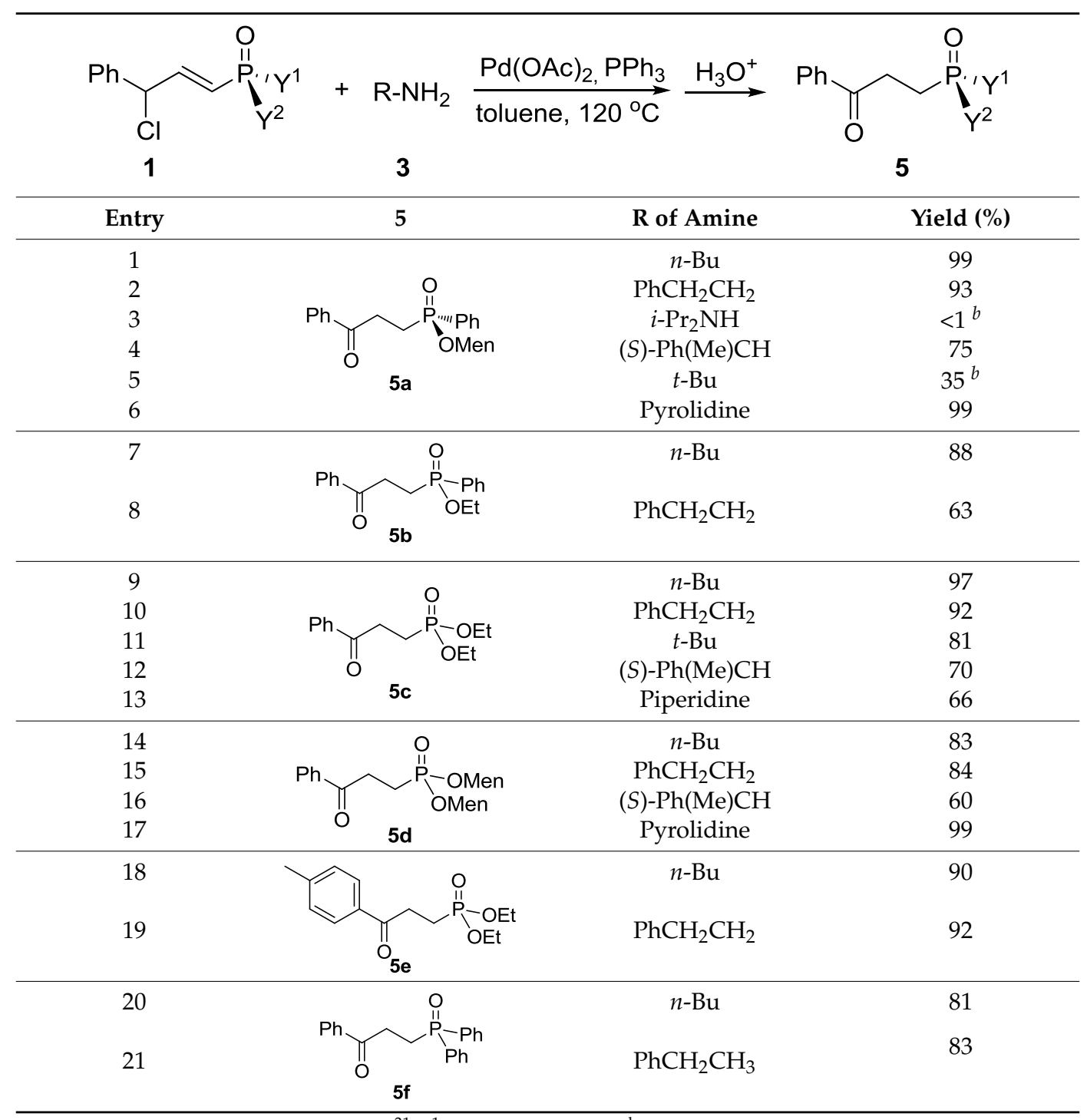

${ }^{a}$ The yield was estimated by a ${ }^{31} \mathrm{P}\left\{{ }^{1} \mathrm{H}\right\}-N M R$ spectrum; ${ }^{\mathrm{b}}$ 4a was formed as the major product.

Secondly, the imine 7 could also be used in situ for the preparation of the $\gamma$-amino phosphorus derivatives 11. In Table 3, various $\mathbf{1 1}$ derivatives were obtained, from the reaction of $\mathbf{1}$ with an amine and subsequent reduction with sodium borohydride, in moderate to excellent yields. The amplifying reaction of $\mathbf{1 f}$ (ca. $1.02 \mathrm{~g}$ ) afforded $\mathbf{1 1 f a}$ with a $59 \%$ isolated yield. When the $P$-stereogenic 1 a reacted with $3 \mathbf{b}$ (2-phenyl ethylamine), 11ab was obtained, which gave two close single peaks at 43.4 and $43.2 \mathrm{ppm}$ on the ${ }^{31} \mathrm{P}-\mathrm{NMR}$ spectrum, in the ratio of around 50:50. The two peaks were ascribed to the two stereoisomers of 11ab deriving from the $\gamma$-stereogenic carbon atom. On the proton NMR spectrum, the $\gamma$-proton was observed as a multi-peak, and the two stereoisomers could not be distinguished. 
Similarly, the two stereoisomers were also observed for $\mathbf{1 1 a c}, \mathbf{1 1 d b}, \mathbf{1 1 d c}$, and $\mathbf{1 1 d d}$. However, only one signal was observed on the ${ }^{31} \mathrm{P}-\mathrm{NMR}$ spectrum for 11aa and 11da (also for 11ga, vide infra) that contained an $\mathrm{N}$-primary butyl. We believe that the two stereoisomers of 11aa and 11da were similarly formed, but their peaks coincided probably due to the weak influences of the butyl on the NMR spectrum (NMR spectrum as seen in SM, Part 3).

Table 3. Preparation of the $\gamma$-amino phosphorous derivatives $\mathbf{1 1}$ via coupling-isomerization and subsequent reduction.

\begin{tabular}{cccccc}
\hline & & & \\
\hline
\end{tabular}

${ }^{\text {a }}$ The yield was estimated by ${ }^{31} \mathrm{P}\left\{{ }^{1} \mathrm{H}\right\}-\mathrm{NMR}$ spectra.

For the reaction of the amines containing sec- or tert-alkyl and aromatic amines, $\mathbf{1 1}$ were not obtained. Instead, the isolation of $\gamma$-hydroxyl phosphorus derivatives $\mathbf{1 2}$ was obtained. We supposed that the imines 7 derived from these amines were instable and hydrolyzed by trace water during the reduction with sodium borohydride; thereby, 12 was formed as the major product (Scheme 4, NMR spectrum as seen in SM, Part 3).

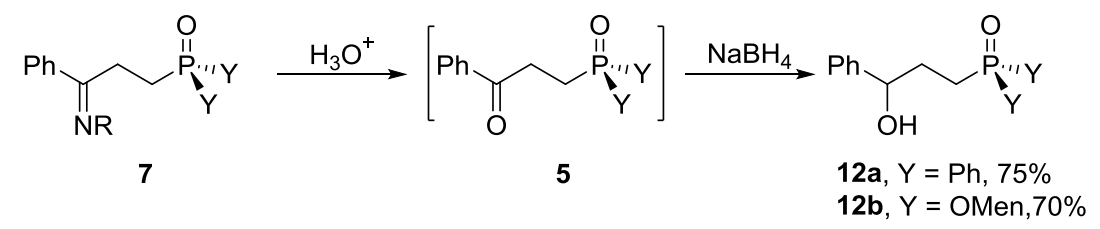

Scheme 4. Preparation of the $\gamma$-hydroxyl phosphorous derivatives 12 (the yield was estimated by a ${ }^{31} \mathrm{P}\left\{{ }^{1} \mathrm{H}\right\}-\mathrm{NMR}$ spectrum).

During the above reaction, only the $\gamma$-functional phosphorus derivatives were obtained. However, for the $P$-stereogenic $\gamma$-chloroallyl menthylphosphine oxide $\mathbf{1 g}$, a mixture of $\alpha$ - and $\gamma$-keto $5 \mathbf{g}$ and $5 \mathbf{g}^{\prime}$ was afforded as observed by the two single peaks at 43.7 and $45.8 \mathrm{ppm}$ on the ${ }^{31} \mathrm{P}-\mathrm{NMR}$ spectrum. The reduction with sodium borohydride gave $\mathbf{1 1}$ and 11' also as a mixture, which indicated that $\alpha$ and $\gamma$-imino $\mathbf{7 g}$ and $\mathbf{7 g}$ ' were formed as intermediates. When $\mathbf{3 b}$ (2-phenyl ethylamine) was used, the structures of the resulting $\mathbf{1 1 g b}$ and $\mathbf{1 1 g b}$ ' were confirmed by an NMR spectrum [46]. 
We found that either $1 \mathrm{~g}$ or $4 \mathrm{~g}$ similarly afforded a mixture of $\alpha$ - and $\gamma$-functional products. As supposed, $1 \mathrm{~g}$ or $\mathbf{4 g}$ formed the allyl palladium complexes $9 \mathrm{~g}$ and $\mathbf{1 0 g}$ (Scheme 5), which were different to the vinyl palladium species of Scheme 3. The reductive elimination of $\alpha$ - or $\gamma$-carbon (route $a$ and route $b$ ) afforded $\mathbf{7 g}$ and $\mathbf{7 \mathbf { g } ^ { \prime }}$, respectively. Compared to $\mathbf{1 a}$ or $\mathbf{1 f}$, the alkoxy or phenyl linked to phosphorus was replaced by an aliphatic menthyl group in 1g. Because of the absence of a $p-d$ or $\pi-d$ interaction for menthyl, the $\alpha$-anion probably was stabilized and the coupling reaction occurred at this position.

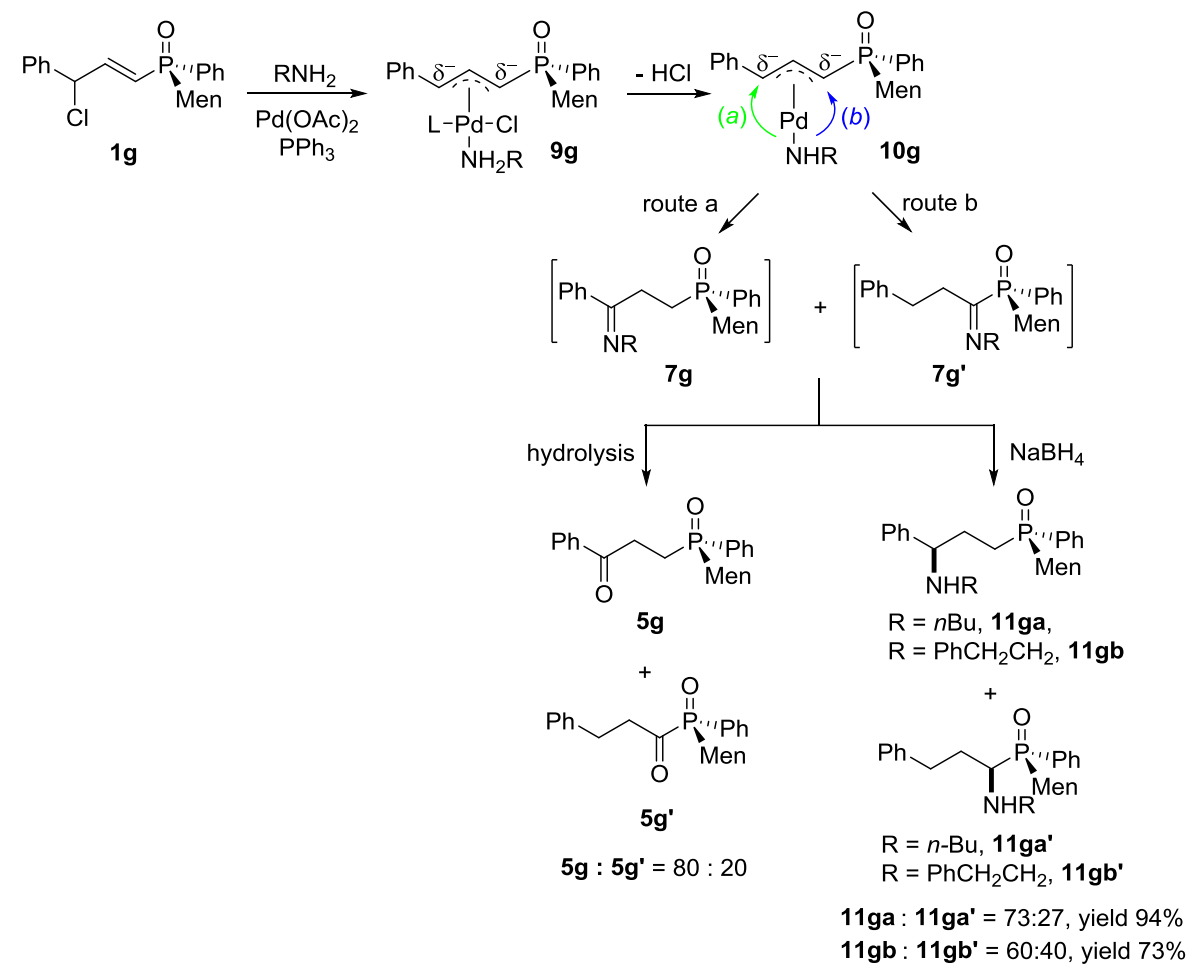

Scheme 5. Reaction of the $P$-stereogenic $1 \mathrm{~g}$ and subsequent conversions (the ratio was estimated by a ${ }^{31} \mathrm{P}\left\{{ }^{1} \mathrm{H}\right\}-\mathrm{NMR}$ spectrum).

When a secondary amine was used, the enamine $\mathbf{6}$ was generated, which could be converted to $\beta$-alkylated products via a Stork reaction [47]. For example, directly heating 6 in situ with acrylonitrile afforded 13a with a $60 \%$ yield. Ethyl acrylate could similarly react with 6 to form $\mathbf{1 3 b}$ (Scheme 6, NMR spectrum as seen in SM, Part 3).

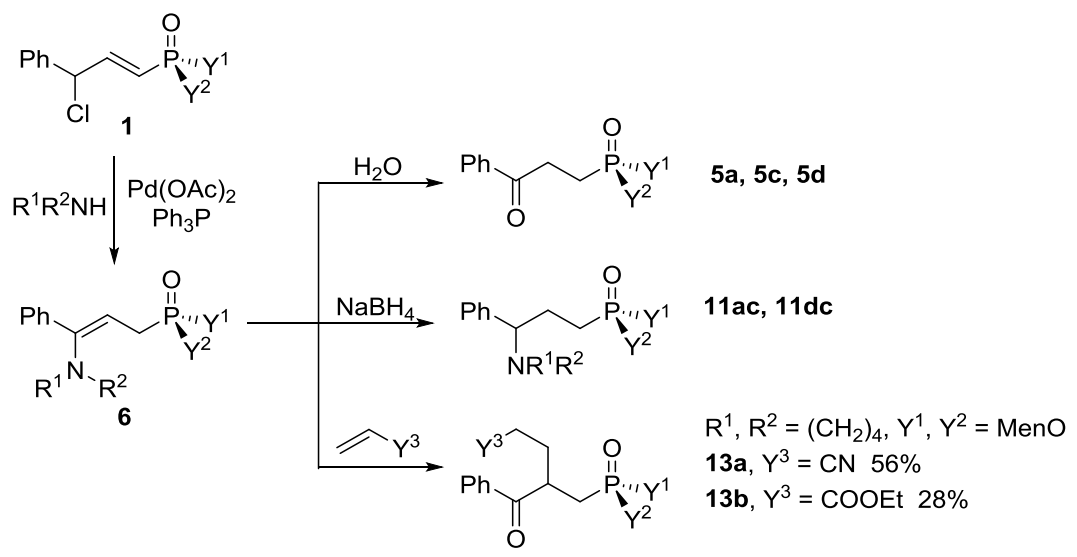

Scheme 6. The preparation of the enamine 6 and the subsequent reactions. 


\section{Materials and Methods}

\subsection{Materials}

${ }^{1} \mathrm{H}-\mathrm{NMR}$ spectra were recorded on a 400-MHz spectrometer (Varian, Palo Alto, CA, USA). The chemical shift for ${ }^{1} \mathrm{H}-\mathrm{NMR}$ spectra is reported (in parts per million) relative to internal tetramethylsilane $\left(\mathrm{Me}_{4} \mathrm{Si}, \delta=0.00 \mathrm{ppm}\right)$ with $\mathrm{CDCl}_{3} .{ }^{13} \mathrm{C}-\mathrm{NMR}$ spectra were recorded at $101 \mathrm{MHz}$. Chemical shifts for ${ }^{13} \mathrm{C}$-NMR spectra are reported (in parts per million) relative to $\mathrm{CDCl}_{3}(\delta=77.0 \mathrm{ppm})$. ${ }^{31} \mathrm{P}-\mathrm{NMR}$ spectra were recorded at $162 \mathrm{MHz}$, and chemical shifts are reported (in parts per million) relative to external $85 \%$ phosphoric acid $(\delta=0.0 \mathrm{ppm})$. TLC plates were visualized by UV (Kang Hua, Shanghai, China). All starting materials were purchased from commercial sources and used as received (Aladdin, Shanghai, China). The solvents were distilled under $\mathrm{N}_{2}$ and dried according to standard procedures. ${ }^{31}$ P-NMR spectra were referenced to phosphoric acid. The NMR yields of the articles are determined by integration of all the resonances in the ${ }^{31} \mathrm{P}$ spectra. The yields obtained by the approach are generally accurate and reproducible.

\subsection{Synthesis}

3.2.1. Addition of H-P Species to Cinnamaldehyde to Form $\alpha$-Hydroxy Allyl Phosphorus Derivatives 2

\section{$\left(S_{P}\right)$-Menthyl-1-hydroxy-3-phenylallyl phenylphosphinate, 2a}

A mixture of $\left(R_{P}\right)$-menthyl phosphinate $(10.0 \mathrm{~g}, 36 \mathrm{mmol})$ and cinnamaldehyde $(5.5 \mathrm{~mL}, 43 \mathrm{mmol})$ was heated at $80^{\circ} \mathrm{C}$ for $5 \mathrm{~h}$ under nitrogen to give a yellow solid, which was recrystallized with dichloromethane/ether to give pure $2 \mathrm{a}$ as a white solid. Yield 68\%, (10.1 g), m.p. 186.0-188.4 ${ }^{\circ} \mathrm{C}$. ${ }^{31} \mathrm{P}-\mathrm{NMR}(162 \mathrm{MHz}, \mathrm{CDCl} 3) \delta=35.67$ (s, 68\%), 35.03 (s, 32\%). ${ }^{1} \mathrm{H}-\mathrm{NMR}(400 \mathrm{MHz}, \mathrm{CDCl} 3) \delta=7.87-7.73$ $(\mathrm{m}, 2 \mathrm{H}), 7.55(\mathrm{q}, J=7.7 \mathrm{~Hz}, 1 \mathrm{H}), 7.49-7.38(\mathrm{~m}, 2 \mathrm{H}), 7.29(\mathrm{dq}, J=16.9,9.7 \mathrm{~Hz}, 5 \mathrm{H}), 6.66-6.49(\mathrm{~m}, 1 \mathrm{H}), 6.27$ $(\mathrm{dtd}, J=55.0,10.8,10.1,5.6 \mathrm{~Hz}, 1 \mathrm{H}), 4.81-4.60(\mathrm{~m}, 1 \mathrm{H}), 4.42(\mathrm{dq}, J=10.7,6.1 \mathrm{~Hz}, 1 \mathrm{H}), 2.30(\mathrm{~d}, J=6.4 \mathrm{~Hz}$, $1 \mathrm{H}), 1.80(\mathrm{~d}, J=11.4 \mathrm{~Hz}, 1 \mathrm{H}), 1.71-1.54(\mathrm{~m}, 2 \mathrm{H}), 1.51-1.38(\mathrm{~m}, 1 \mathrm{H}), 1.31(\mathrm{~s}, 1 \mathrm{H}), 1.11-0.95(\mathrm{~m}, 2 \mathrm{H}), 0.93$ $(\mathrm{d}, J=7.0 \mathrm{~Hz}, 2 \mathrm{H}), 0.88-0.82(\mathrm{~m}, 3 \mathrm{H}), 0.79(\mathrm{~d}, J=6.9 \mathrm{~Hz}, 2 \mathrm{H}), 0.75(\mathrm{dd}, J=6.4,3.2 \mathrm{~Hz}, 4 \mathrm{H})$.

Ethyl 1-hydroxy-3-phenylallyl phenylphosphinate, 2b

A solution of dichlorophenylphosphine $(5 \mathrm{~mL}, 37 \mathrm{mmol})$ in acetonitrile $(10 \mathrm{~mL})$ was added dropwise to ethanol $(4.7 \mathrm{~mL}, 81 \mathrm{mmol})$ with cooling in an ice bath. After the mixture was warmed to room temperature, potassium carbonate $(6.4 \mathrm{~g}, 46 \mathrm{mmol})$ and cinnamaldehyde $(5.1 \mathrm{~mL}, 40.7 \mathrm{mmol})$ were added. The resulting mixture was stirred at room temperature for $14 \mathrm{~h}$. A saturated solution of ammonium chloride $(10 \mathrm{~mL})$ was added and the mixture was extracted with dichloromethane $(3 \times 30 \mathrm{~mL})$ then washed with water $(3 \times 20 \mathrm{~mL})$. The combined organic layer was dried over anhydrous magnesium sulfate and concentrated in vacuo to give the crude product, which was recrystallized with ether to give pure $2 \mathrm{~b}$ as a white solid. Yield 55\%, (6.1 g), m.p. 137.6-141.7 ${ }^{\circ} \mathrm{C}$. ${ }^{31} \mathrm{P}-\mathrm{NMR}\left(162 \mathrm{MHz}, \mathrm{CDCl}_{3}\right) \delta=33.62$ (s, 32\%), 32.73 (s, 68\%). ${ }^{1} \mathrm{H}-\mathrm{NMR}\left(400 \mathrm{MHz}, \mathrm{CDCl}_{3}\right) \delta=7.87-7.74$ $(\mathrm{m}, 2 \mathrm{H}), 7.56(\mathrm{dd}, J=14.6,7.3 \mathrm{~Hz}, 1 \mathrm{H}), 7.51-7.39(\mathrm{~m}, 2 \mathrm{H}), 7.39-7.19(\mathrm{~m}, 5 \mathrm{H}), 6.65-6.54(\mathrm{~m}, 1 \mathrm{H}), 6.35-6.17$ $(\mathrm{m}, 1 \mathrm{H}), 4.78(\mathrm{~d}, J=25.4 \mathrm{~Hz}, 1 \mathrm{H}), 4.16(\mathrm{dddd}, J=24.8,21.1,12.2,6.7 \mathrm{~Hz}, 2 \mathrm{H}), 1.35(\mathrm{td}, J=7.0,2.9 \mathrm{~Hz}, 3 \mathrm{H})$.

\section{Diethyl 1-hydroxy-3-phenylallylphosphonate, 2c}

To a solution of diethyl phosphite $(3.6 \mathrm{~mL}, 28 \mathrm{mmol})$ in acetonitrile $(10 \mathrm{~mL})$, potassium carbonate $(5.8 \mathrm{~g}, 42 \mathrm{mmol})$ and cinnamaldehyde $(4.2 \mathrm{~mL}, 33.6 \mathrm{mmol})$ were added at room temperature. The mixture was stirred for $13 \mathrm{~h}$ at the same temperature. A saturated solution of ammonium chloride $(10 \mathrm{~mL})$ was added and the mixture was extracted with dichloromethane $(3 \times 30 \mathrm{~mL})$ then washed with water $(3 \times 20 \mathrm{~mL})$. The combined organic layer was dried over anhydrous magnesium sulfate and concentrated in vacuo. The crude product was recrystallized with ether to give pure $2 \mathrm{c}$ as a white solid. Yield 87\%, 9.1 g, m.p. 98.7-100.0 ${ }^{\circ} \mathrm{C} .{ }^{31} \mathrm{P}-\mathrm{NMR}\left(162 \mathrm{MHz}, \mathrm{CDCl}_{3}\right) \delta=21.50$ (s). ${ }^{1} \mathrm{H}-\mathrm{NMR}$ 
$\left(400 \mathrm{MHz}, \mathrm{CDCl}_{3}\right) \delta=7.40(\mathrm{~d}, J=7.4 \mathrm{~Hz}, 2 \mathrm{H}), 7.31(\mathrm{t}, J=7.4 \mathrm{~Hz}, 2 \mathrm{H}), 7.25(\mathrm{~d}, J=5.0 \mathrm{~Hz}, 1 \mathrm{H}), 6.78(\mathrm{dd}$, $J=16.1,4.1 \mathrm{~Hz}, 1 \mathrm{H}), 6.36-6.27(\mathrm{~m}, 1 \mathrm{H}), 4.66(\mathrm{dd}, J=13.0,6.2 \mathrm{~Hz}, 1 \mathrm{H}), 4.23-4.15(\mathrm{~m}, 4 \mathrm{H}), 1.33(\mathrm{td}, J=7.0$, $4.2 \mathrm{~Hz}, 6 \mathrm{H})$.

Dimenthyl 1-hydroxy-3-phenylallylphosphonate, $\mathbf{2 d}$

Compound $\mathbf{2 d}$ was prepared according a similar procedure to $\mathbf{2 c}$, which was purified by chromatography with $R_{f}=0.22$ (silica gel, petroleum ether). Pure $\mathbf{2 d}$ was obtained as yellow oil. Yield 60\% (4.4 g). ${ }^{31} \mathrm{P}-\mathrm{NMR}(162 \mathrm{MHz}, \mathrm{CDCl} 3) \delta=19.95$ (s, 52\%), 19.53 (s, 48\%). ${ }^{1} \mathrm{H}-\mathrm{NMR}(400 \mathrm{MHz}$, $\mathrm{CDCl} 3) \delta=7.41-7.35(\mathrm{~m}, 2 \mathrm{H}), 7.31(\mathrm{t}, J=6.8 \mathrm{~Hz}, 2 \mathrm{H}), 7.24(\mathrm{~d}, J=6.7 \mathrm{~Hz}, 1 \mathrm{H}), 6.82-6.73(\mathrm{~m}, 1 \mathrm{H}), 6.33(\mathrm{dt}$, $J=16.0,5.1 \mathrm{~Hz}, 1 \mathrm{H}), 4.62(\mathrm{~d}, J=14.2 \mathrm{~Hz}, 1 \mathrm{H}), 4.36-4.21(\mathrm{~m}, 2 \mathrm{H}), 2.41-2.10(\mathrm{~m}, 4 \mathrm{H}), 1.66(\mathrm{~d}, J=7.5 \mathrm{~Hz}$, $4 \mathrm{H}), 1.50-1.29(\mathrm{~m}, 4 \mathrm{H}), 1.22-1.09(\mathrm{~m}, 2 \mathrm{H}), 1.06-0.96(\mathrm{~m}, 2 \mathrm{H}), 0.89(\mathrm{dt}, J=10.8,6.7 \mathrm{~Hz}, 12 \mathrm{H}), 0.85-0.77$ $(\mathrm{m}, 9 \mathrm{H})$.

Diethyl 1-hydroxy-3-p-tolyl allylphosphonate, 2e

Compound 2e was prepared according a similar procedure to $\mathbf{2 c}$, which was recrystallized with ether. The pure $2 \mathbf{e}$ was obtained as a white solid. Yield $80 \%(6.4 \mathrm{~g})$, m.p. $108.1-113.0^{\circ} \mathrm{C} .{ }^{31} \mathrm{P}-\mathrm{NMR}$ $(162 \mathrm{MHz}, \mathrm{CDCl} 3) \delta=21.45$ (s). ${ }^{1} \mathrm{H}-\mathrm{NMR}(400 \mathrm{MHz}, \mathrm{CDCl} 3) \delta=7.30(\mathrm{~d}, J=7.7 \mathrm{~Hz}, 2 \mathrm{H}), 7.13(\mathrm{~d}$, $J=7.7 \mathrm{~Hz}, 2 \mathrm{H}), 6.75(\mathrm{~d}, J=16.1 \mathrm{~Hz}, 1 \mathrm{H}), 6.26(\mathrm{dt}, J=15.9,5.9 \mathrm{~Hz}, 1 \mathrm{H}), 4.71-4.60(\mathrm{~m}, 1 \mathrm{H}), 4.19(\mathrm{~m}, 4 \mathrm{H})$, $2.34(\mathrm{~s}, 3 \mathrm{H}), 1.34(\mathrm{t}, J=7.1 \mathrm{~Hz}, 6 \mathrm{H})$.

\section{Diphenyl 1-hydroxy-3-phenylallylphosphine oxide, $\mathbf{2 f}$}

To a solution of chlorodiphenyl phosphine $(6 \mathrm{~mL}, 29 \mathrm{mmol})$ in acetonitrile $(20 \mathrm{~mL})$, water $(0.5 \mathrm{~mL})$ was added dropwise with cooling in an ice bath, then potassium carbonate $(6.0 \mathrm{~g}, 43.5 \mathrm{mmol})$ and cinnamaldehyde $(4.4 \mathrm{~mL}, 34.8 \mathrm{mmol})$ were added. The resulting mixture was stirred at room temperature for $18 \mathrm{~h}$. A saturated solution of ammonium chloride $(10 \mathrm{~mL})$ was added and the resulting pink solid was collected by filtration and recrystallized with ethanol/ether to give pure $\mathbf{2} \mathbf{f}$ as a white solid. Yield 41\% (3.9 g), m.p. 168.9-170.3 ${ }^{\circ} \mathrm{C} .{ }^{31} \mathrm{P}-\mathrm{NMR}\left(162 \mathrm{MHz}, \mathrm{CDCl}_{3}\right) \delta=26.31$ (s). ${ }^{1} \mathrm{H}-\mathrm{NMR}$ $\left(400 \mathrm{MHz}, \mathrm{CDCl}_{3}\right) \delta=7.91-7.80(\mathrm{~m}, 4 \mathrm{H}), 7.55(\mathrm{dd}, J=15.1,7.7 \mathrm{~Hz}, 2 \mathrm{H}), 7.50-7.43(\mathrm{~m}, 4 \mathrm{H}), 7.27-7.20(\mathrm{~m}$, $5 \mathrm{H}), 6.58(\mathrm{dd}, J=16.0,4.4 \mathrm{~Hz}, 1 \mathrm{H}), 6.31-6.23(\mathrm{~m}, 1 \mathrm{H}), 5.16(\mathrm{t}, J=6.4 \mathrm{~Hz}, 1 \mathrm{H})$.

\section{$\left(S_{P}\right)$-Menthyl 1-hydroxy-3-phenylallylphenylphosphine oxide, $\mathbf{2 g}$}

Compound $\mathbf{2 g}$ was prepared according a similar procedure to $\mathbf{2 a}$, which was recrystallized with ether, and was obtained as a white solid. Yield 54\% (8.1 g), m.p. $151.5-156.4{ }^{\circ} \mathrm{C} .{ }^{31} \mathrm{P}-\mathrm{NMR}(162 \mathrm{MHz}$, $\left.\mathrm{CDCl}_{3}\right) \delta=40.12(\mathrm{~s}, 58 \%), 36.47(\mathrm{~s}, 42 \%) .{ }^{1} \mathrm{H}-\mathrm{NMR}\left(400 \mathrm{MHz}, \mathrm{CDCl}_{3}\right) \delta=7.75(\mathrm{dt}, J=42.3,8.9 \mathrm{~Hz}$, 2H), 7.56-7.36 (m, 3H), 7.23-7.12 (m, 5H), $6.44(\mathrm{dd}, J=59.9,16.5 \mathrm{~Hz}, 1 \mathrm{H}), 6.17-6.03(\mathrm{~m}, 1 \mathrm{H}), 4.94(\mathrm{~d}$, $J=23.5 \mathrm{~Hz}, 1 \mathrm{H}), 2.52-2.20(\mathrm{~m}, 2 \mathrm{H}), 1.95-1.63(\mathrm{~m}, 4 \mathrm{H}), 1.46(\mathrm{~d}, J=30.6 \mathrm{~Hz}, 2 \mathrm{H}), 1.18-1.00(\mathrm{~m}, 2 \mathrm{H}), 0.97$ $(\mathrm{d}, J=5.9 \mathrm{~Hz}, 1 \mathrm{H}), 0.91(\mathrm{t}, J=5.9 \mathrm{~Hz}, 3 \mathrm{H}), 0.76(\mathrm{~d}, J=6.8 \mathrm{~Hz}, 1 \mathrm{H}), 0.70(\mathrm{~d}, J=6.8 \mathrm{~Hz}, 2 \mathrm{H}), 0.29$ (d, $J=6.5 \mathrm{~Hz}, 1 \mathrm{H})$.

\subsubsection{Chlorination of $\alpha$-Hydroxy Allyl Phosphorus Derivatives $\mathbf{2}$ to Form 1a-1g}

$\left(S_{P}\right)$-Menthyl 3-chloro-3-phenylprop-1-en-1-yl phenylphosphinate, 1a

To an ice-cooled solution of $2 \mathrm{a}(2.0 \mathrm{~g}, 4.8 \mathrm{mmol})$ and pyridine $(0.6 \mathrm{~mL}, 7.2 \mathrm{mmol})$ in dichloromethane $(10 \mathrm{~mL})$, thionyl chloride $(0.5 \mathrm{~mL}, 7.2 \mathrm{mmol})$ was added dropwise. The mixture was allowed to warm to room temperature slowly over $5 \mathrm{~h}$. Water $(5 \mathrm{~mL})$ was added and the aqueous layer was extracted with dichloromethane $(3 \times 10 \mathrm{~mL})$. The combined organic layer was dried over anhydrous magnesium sulfate and concentrated in vacuo. The crude product was recrystallized with petroleum ether $\left(30-60{ }^{\circ} \mathrm{C}\right)$ to give pure $1 \mathrm{a}$ as a white solid. Yield $97 \%(2.1 \mathrm{~g})$, m.p. $148.7-154.1^{\circ} \mathrm{C}$. ${ }^{31} \mathrm{P}-\mathrm{NMR}\left(162 \mathrm{MHz}, \mathrm{CDCl}_{3}\right) \delta=27.45(\mathrm{~s}, 23 \%), 27.25$ (s, 77\%). ${ }^{1} \mathrm{H}-\mathrm{NMR}\left(400 \mathrm{MHz}, \mathrm{CDCl}_{3}\right) \delta=7.83-7.73$ $(\mathrm{m}, 2 \mathrm{H}), 7.57-7.51(\mathrm{~m}, 1 \mathrm{H}), 7.47(\mathrm{dt}, J=7.2,3.6 \mathrm{~Hz}, 2 \mathrm{H}), 7.38-7.29(\mathrm{~m}, 5 \mathrm{H}), 6.98$ (dddd, $J=19.1,16.6$, $5.8,2.8 \mathrm{~Hz}, 1 \mathrm{H}), 6.19(\mathrm{dd}, J=20.5,16.6 \mathrm{~Hz}, 1 \mathrm{H}), 5.53(\mathrm{~d}, J=5.6 \mathrm{~Hz}, 1 \mathrm{H}), 4.31-4.16(\mathrm{~m}, 1 \mathrm{H}), 2.10(\mathrm{~d}$, 
$J=13.6 \mathrm{~Hz}, 2 \mathrm{H}), 2.03(\mathrm{~s}, 1 \mathrm{H}), 1.65(\mathrm{~d}, J=6.9 \mathrm{~Hz}, 2 \mathrm{H}), 1.41(\mathrm{~d}, J=9.4 \mathrm{~Hz}, 2 \mathrm{H}), 1.20-1.09(\mathrm{~m}, 1 \mathrm{H}), 0.93$ $(\mathrm{dd}, J=9.1,7.1 \mathrm{~Hz}, 3 \mathrm{H}), 0.86-0.81(\mathrm{~m}, 4 \mathrm{H}), 0.80(\mathrm{~s}, 1 \mathrm{H}), 0.75(\mathrm{~d}, J=6.9 \mathrm{~Hz}, 2 \mathrm{H})$.

Ethyl 3-chloro-3-phenylprop-1-en-1-ylphenylphosphinate, $\mathbf{1 b}$

The compound $\mathbf{1 b}$ was prepared similar to $\mathbf{1 a}$, which was purified with flash column chromatography with $R_{f}=0.46$ (silica gel, petroleum ether/ethyl acetate $=2: 1$ as eluent), and was obtained as a yellow oil. Yield 70\% (1.5 g). ${ }^{31} \mathrm{P}-\mathrm{NMR}\left(162 \mathrm{MHz}, \mathrm{CDCl}_{3}\right) \delta=33.18(\mathrm{~s}, 50 \%), 33.10(\mathrm{~s}$, $50 \%) .{ }^{1} \mathrm{H}-\mathrm{NMR}\left(400 \mathrm{MHz}, \mathrm{CDCl}_{3}\right) \delta=7.83-7.63(\mathrm{~m}, 2 \mathrm{H}), 7.55-7.43(\mathrm{~m}, 1 \mathrm{H}), 7.43-7.33(\mathrm{~m}, 2 \mathrm{H}), 7.35-7.11$ $(\mathrm{m}, 5 \mathrm{H}), 6.87(\mathrm{dddd}, J=19.1,16.7,5.8,2.3 \mathrm{~Hz}, 1 \mathrm{H}), 6.22-6.05(\mathrm{~m}, 1 \mathrm{H}), 5.50-5.40(\mathrm{~m}, 1 \mathrm{H}), 4.07-3.83(\mathrm{~m}$, $2 \mathrm{H}), 1.32-1.18(\mathrm{~m}, 3 \mathrm{H})$.

Diethyl 3-chloro-3-phenylprop-1-en-1-ylphosphonate, 1c

The compound 1c was prepared similar to 1a, which was purified with flash column chromatography with $R_{f}=0.52$ (silica gel, petroleum ether/ethyl acetate $=3: 1$ as eluent), and was obtained as a yellow oil. Yield 65\% (1.4 g). ${ }^{31} \mathrm{P}-\mathrm{NMR}\left(162 \mathrm{MHz}, \mathrm{CDCl}_{3}\right) \delta=16.82(\mathrm{~s}) .{ }^{1} \mathrm{H}-\mathrm{NMR}$ $\left(400 \mathrm{MHz}, \mathrm{CDCl}_{3}\right) \delta=7.47-7.31(\mathrm{~m}, 5 \mathrm{H}), 6.96(\mathrm{ddd}, J=22.5,16.7,5.9 \mathrm{~Hz}, 1 \mathrm{H}), 6.08-5.91(\mathrm{~m}, 1 \mathrm{H}), 5.52$ $(\mathrm{d}, J=4.6 \mathrm{~Hz}, 1 \mathrm{H}), 4.21-3.98(\mathrm{~m}, 4 \mathrm{H}), 1.40-1.23(\mathrm{~m}, 6 \mathrm{H}) .{ }^{13} \mathrm{C}-\mathrm{NMR}\left(101 \mathrm{MHz}, \mathrm{CDCl}_{3}\right) \delta=149.0(\mathrm{~d}$, $J=7.0 \mathrm{~Hz}), 138.1(\mathrm{~s}), 128.9(\mathrm{~d}, J=1.4 \mathrm{~Hz}), 127.5(\mathrm{~s}), 120.1(\mathrm{~s}), 118.2(\mathrm{~s}), 62.0(\mathrm{dd}, J=5.6,2.9 \mathrm{~Hz}), 61.8(\mathrm{~d}$, $J=25.2 \mathrm{~Hz}), 16.3(\mathrm{~d}, J=6.3 \mathrm{~Hz})$. HRMS $\left(\mathrm{ESI}^{+}\right)$Calcd. for $\mathrm{C}_{13} \mathrm{H}_{18} \mathrm{ClO}_{3} \mathrm{P}\left[\mathrm{M}+\mathrm{Na}^{+}\right]: 311.0580$, Found: 311.0587.

\section{Dimenthyl 3-chloro-3-phenylprop-1-en-1-ylphosphonate, 1d}

The compound $\mathbf{1 d}$ was prepared similar to $\mathbf{1 a}$, which was purified with flash column chromatography with $R_{f}=0.48$ (silica gel, petroleum ether/ethyl acetate $=5: 1$ as eluent), and was obtained as a yellow oil. Yield 92\% (1.9 g). ${ }^{31} \mathrm{P}-\mathrm{NMR}\left(162 \mathrm{MHz}, \mathrm{CDCl}_{3}\right) \delta=14.80(\mathrm{~s}, 51 \%), 14.65$ (s, 49\%). ${ }^{1} \mathrm{H}-\mathrm{NMR}\left(400 \mathrm{MHz}, \mathrm{CDCl}_{3}\right) \delta=7.36(\mathrm{~d}, J=2.3 \mathrm{~Hz}, 4 \mathrm{H}), 7.25(\mathrm{dd}, J=13.4,5.9 \mathrm{~Hz}, 1 \mathrm{H}), 7.00-6.86$ $(\mathrm{m}, 1 \mathrm{H}), 6.05-5.88(\mathrm{~m}, 1 \mathrm{H}), 5.50(\mathrm{~d}, J=5.0 \mathrm{~Hz}, 1 \mathrm{H}), 4.34-4.04(\mathrm{~m}, 2 \mathrm{H}), 2.19(\mathrm{dd}, J=40.1,9.0 \mathrm{~Hz}, 3 \mathrm{H})$, $1.98(\mathrm{~d}, J=5.8 \mathrm{~Hz}, 1 \mathrm{H}), 1.64(\mathrm{~d}, J=8.6 \mathrm{~Hz}, 4 \mathrm{H}), 1.52-1.24(\mathrm{~m}, 4 \mathrm{H}), 1.22-1.07(\mathrm{~m}, 2 \mathrm{H}), 1.07-0.76(\mathrm{~m}, 20 \mathrm{H})$, $0.73(\mathrm{~d}, J=6.9 \mathrm{~Hz}, 1 \mathrm{H}), 0.66(\mathrm{~d}, J=6.9 \mathrm{~Hz}, 1 \mathrm{H}) .{ }^{13} \mathrm{C}-\mathrm{NMR}\left(101 \mathrm{MHz}, \mathrm{CDCl}_{3}\right) \delta=147.8(\mathrm{dd}, J=14.9$, $7.5 \mathrm{~Hz}), 138.4(\mathrm{~d}, J=3.8 \mathrm{~Hz}), 128.8(\mathrm{~d}, J=4.4 \mathrm{~Hz}), 127.5(\mathrm{~d}, J=5.0 \mathrm{~Hz}), 121.2(\mathrm{~d}, J=188.5 \mathrm{~Hz}), 77.7(\mathrm{dd}$, $J=6.7,3.8 \mathrm{~Hz}), 62.0(\mathrm{dd}, J=25.5,3.4 \mathrm{~Hz}), 48.4(\mathrm{dd}, J=6.7,3.6 \mathrm{~Hz}), 43.49(\mathrm{~s}), 43.1(\mathrm{~d}, J=4.4 \mathrm{~Hz}), 34.0(\mathrm{~s})$, 31.7-31.3 (m), $25.5(\mathrm{~d}, J=6.7 \mathrm{~Hz}), 22.8(\mathrm{~s}), 21.9(\mathrm{~d}, J=3.1 \mathrm{~Hz}), 20.9(\mathrm{~d}, J=2.5 \mathrm{~Hz}), 15.8(\mathrm{~d}, J=1.4 \mathrm{~Hz})$, $15.6(\mathrm{~d}, J=13.1 \mathrm{~Hz})$. HRMS $\left(\mathrm{ESI}^{+}\right)$Calcd. for $\mathrm{C}_{29} \mathrm{H}_{46} \mathrm{ClO}_{3} \mathrm{P}\left[\mathrm{M}+\mathrm{Na}^{+}\right]$: 531.2771, Found: 531.2766.

Diethyl 3-chloro-3-p-tolylprop-1-en-1-ylphosphonate, $\mathbf{1 e}$

The compound 1e was prepared similar to $\mathbf{1 a}$, which was purified with flash column chromatography (silica gel, petroleum ether/ethyl acetate $=1: 1$ as eluent), and was obtained as a yellow oil. Yield 65\% (1.4 g). ${ }^{31} \mathrm{P}-\mathrm{NMR}\left(162 \mathrm{MHz}, \mathrm{CDCl}_{3}\right) \delta=16.93$ (s). ${ }^{1} \mathrm{H}-\mathrm{NMR}\left(400 \mathrm{MHz}, \mathrm{CDCl}_{3}\right)$ $\delta=7.27-7.22(\mathrm{~m}, 2 \mathrm{H}), 7.18(\mathrm{~d}, J=7.8 \mathrm{~Hz}, 2 \mathrm{H}), 6.95(\mathrm{ddd}, J=21.6,16.7,5.9 \mathrm{~Hz}, 1 \mathrm{H}), 6.03-5.90(\mathrm{~m}, 1 \mathrm{H})$, $5.50(\mathrm{~d}, J=5.6 \mathrm{~Hz}, 1 \mathrm{H}), 4.09(\mathrm{dq}, J=12.1,6.5,6.0 \mathrm{~Hz}, 4 \mathrm{H}), 2.35(\mathrm{~s}, 3 \mathrm{H}), 1.34(\mathrm{~m}, 6 \mathrm{H}) .{ }^{13} \mathrm{C}-\mathrm{NMR}(101 \mathrm{MHz}$, $\left.\mathrm{CDCl}_{3}\right) \delta=150.5(\mathrm{dd}, J=250.0,6.2 \mathrm{~Hz}), 138.4(\mathrm{~d}, J=89.2 \mathrm{~Hz}), 135.3(\mathrm{~d}, J=24.5 \mathrm{~Hz}), 129.5(\mathrm{~s}), 129.3(\mathrm{~s})$, $129.1(\mathrm{~s}), 127.5(\mathrm{~s}), 127.1(\mathrm{~s}), 126.8(\mathrm{~s}), 126.5(\mathrm{~s}), 117.4(\mathrm{dd}, J=300.6,187.1 \mathrm{~Hz}), 82.9(\mathrm{~d}, J=21.3 \mathrm{~Hz}), 62.0$ $(\mathrm{dd}, J=6.5,3.5 \mathrm{~Hz}), 61.7(\mathrm{~d}, J=6.1 \mathrm{~Hz}), 56.5(\mathrm{~s}), 21.1(\mathrm{~d}, J=1.2 \mathrm{~Hz}), 16.3(\mathrm{~d}, J=6.3 \mathrm{~Hz})$. HRMS $\left(\mathrm{ESI}^{+}\right)$ Calcd. for $\mathrm{C}_{14} \mathrm{H}_{20} \mathrm{ClO}_{3} \mathrm{P}\left[\mathrm{M}+\mathrm{Na}^{+}\right.$]: 325.0736, Found: 325.0746.

Diphenyl 3-chloro-3-phenylprop-1-en-1-ylphosphine oxide, 1 f

The compound 1f was prepared similar to 1a, which was recrystallized with dichloromethane/ether, and was obtained as a yellow solid. Yield $90 \%(1.9 \mathrm{~g})$, m.p. $109.2-112.4{ }^{\circ} \mathrm{C}$. ${ }^{31} \mathrm{P}-\mathrm{NMR}\left(162 \mathrm{MHz}, \mathrm{CDCl}_{3}\right) \delta=18.16$ (s). ${ }^{1} \mathrm{H}-\mathrm{NMR}\left(400 \mathrm{MHz}, \mathrm{CDCl}_{3}\right) \delta=7.73-7.64(\mathrm{~m}, 4 \mathrm{H}), 7.59-7.52$ $(\mathrm{m}, 2 \mathrm{H}), 7.48(\mathrm{dddd}, J=8.4,7.0,4.5,2.3 \mathrm{~Hz}, 4 \mathrm{H}), 7.39-7.31(\mathrm{~m}, 5 \mathrm{H}), 6.99(\mathrm{ddd}, J=18.2,16.6,5.6 \mathrm{~Hz}, 1 \mathrm{H})$, $6.62(\mathrm{ddd}, J=22.5,16.6,1.5 \mathrm{~Hz}, 1 \mathrm{H}), 5.62(\mathrm{dt}, J=5.6,2.0 \mathrm{~Hz}, 1 \mathrm{H}) .{ }^{13} \mathrm{C}-\mathrm{NMR}\left(101 \mathrm{MHz}, \mathrm{CDCl}_{3}\right) \delta=148.2$ 
$(\mathrm{d}, J=4.1 \mathrm{~Hz}), 138.3(\mathrm{~s}), 132.1(\mathrm{~d}, J=2.5 \mathrm{~Hz}), 131.3(\mathrm{dd}, J=10.0,1.5 \mathrm{~Hz}), 129.0(\mathrm{~s}), 128.8(\mathrm{~d}, J=1.4 \mathrm{~Hz})$, $128.7(\mathrm{~d}, J=1.4 \mathrm{~Hz}), 127.6(\mathrm{~s}), 124.3(\mathrm{~d}, J=99.0 \mathrm{~Hz}), 65.8(\mathrm{~s}), 62.3(\mathrm{~d}, J=18.7 \mathrm{~Hz}), 15.3(\mathrm{~s})$. HRMS $^{\left(\mathrm{ESI}^{+}\right)}$ Calcd. for $\mathrm{C}_{21} \mathrm{H}_{18} \mathrm{ClOP}\left[\mathrm{M}+\mathrm{Na}^{+}\right.$]: 375.0681, Found: 375.0692 .

$\left(S_{P}\right)$-Menthyl-3-chloro-3-phenylprop-1-en-1-yl phenylphosphine oxide, $\mathbf{1 g}$

The compound $1 \mathrm{~g}$ was prepared similar to $1 \mathrm{a}$, which was recrystallized with ether, and was obtained as a white solid. Yield 80\% (1.6 g), m.p. 138.9-143.6 ${ }^{\circ} \mathrm{C} .{ }^{31} \mathrm{P}-\mathrm{NMR}\left(162 \mathrm{MHz}, \mathrm{CDCl}_{3}\right) \delta=32.80$ $(\mathrm{s}, 32 \%), 32.67(\mathrm{~s}, 68 \%) .{ }^{1} \mathrm{H}-\mathrm{NMR} \delta=7.77-7.65(\mathrm{~m}, 2 \mathrm{H}), 7.48(\mathrm{~s}, 3 \mathrm{H}), 7.34(\mathrm{~d}, J=8.2 \mathrm{~Hz}, 5 \mathrm{H}), 6.96(\mathrm{td}$, $J=16.6,5.5 \mathrm{~Hz}, 1 \mathrm{H}), 6.56-6.28(\mathrm{~m}, 1 \mathrm{H}), 5.60(\mathrm{~d}, J=5.4 \mathrm{~Hz}, 1 \mathrm{H}), 2.15(\mathrm{~s}, 1 \mathrm{H}), 2.07-1.94(\mathrm{~m}, 1 \mathrm{H}), 1.80-1.48$ $(\mathrm{m}, 4 \mathrm{H}), 1.02(\mathrm{dq}, J=26.1,14.2,13.3 \mathrm{~Hz}, 2 \mathrm{H}), 0.89(\mathrm{~d}, J=6.5 \mathrm{~Hz}, 2 \mathrm{H}), 0.84(\mathrm{~d}, J=6.5 \mathrm{~Hz}, 3 \mathrm{H}), 0.78(\mathrm{~d}$, $J=6.6 \mathrm{~Hz}, 2 \mathrm{H}), 0.48(\mathrm{t}, J=6.2 \mathrm{~Hz}, 3 \mathrm{H}) .{ }^{13} \mathrm{C}-\mathrm{NMR}\left(101 \mathrm{MHz}, \mathrm{CDCl}_{3}\right) \delta=146.6(\mathrm{dd}, J=140.6,2.5 \mathrm{~Hz})$, $138.7(\mathrm{~d}, J=7.0 \mathrm{~Hz}), 131.3(\mathrm{~d}, J=2.6 \mathrm{~Hz}), 130.3(\mathrm{dd}, J=8.6,4.7 \mathrm{~Hz}), 129.0(\mathrm{~s}), 128.8(\mathrm{~d}, J=5.1 \mathrm{~Hz}), 128.6$ $(\mathrm{dd}, J=11.4,6.1 \mathrm{~Hz}), 127.6(\mathrm{~d}, J=1.5 \mathrm{~Hz}), 62.8-62.2(\mathrm{~m}), 43.7-43.0(\mathrm{~m}), 41.0(\mathrm{dd}, J=71.6,30.3 \mathrm{~Hz}), 35.6$ (d, $J=95.2 \mathrm{~Hz}), 34.3(\mathrm{~s}), 33.2(\mathrm{dd}, J=13.7,11.4 \mathrm{~Hz}), 28.3(\mathrm{dd}, J=7.6,2.9 \mathrm{~Hz}), 24.6(\mathrm{~d}, J=12.8 \mathrm{~Hz}), 22.5$ (s), $21.5(\mathrm{~d}, J=6.3 \mathrm{~Hz}), 15.4(\mathrm{~d}, J=42.7 \mathrm{~Hz}) \cdot \mathrm{HRMS}\left(\mathrm{ESI}^{+}\right)$Calcd. for $\mathrm{C}_{25} \mathrm{H}_{32} \mathrm{ClOP}\left[\mathrm{M}+\mathrm{Na}^{+}\right]: 437.1777$, Found: 437.1773 .

\subsubsection{The Preparation of ( $\left.\mathrm{S}_{\mathrm{P}}\right)$-Menthyl 3-Chloro-3-Phenylallyl Phenyl Phosphine Oxide, $\mathbf{4 g}$}

The compound $1 \mathrm{~g}$ ( $50 \mathrm{mg}, 0.131 \mathrm{mmol})$ was dissolved in toluene $(0.3 \mathrm{~mL})$ and then heated at 120 ${ }^{\circ} \mathrm{C}$ for $14 \mathrm{~h}$. After removing the solvent in vacuo, $4 \mathrm{~g}$ was obtained as a yellow oil. Yield $98 \%(49 \mathrm{mg})$. ${ }^{31} \mathrm{P}-\mathrm{NMR}\left(162 \mathrm{MHz}, \mathrm{CDCl}_{3}\right) \delta=41.60$ (s). ${ }^{1} \mathrm{H}-\mathrm{NMR}\left(400 \mathrm{MHz}, \mathrm{CDCl}_{3}\right) \delta=7.78-7.70(\mathrm{~m}, 2 \mathrm{H}), 7.45$ $(\mathrm{s}, 3 \mathrm{H}), 7.40-7.33(\mathrm{~m}, 2 \mathrm{H}), 7.27(\mathrm{dd}, J=3.9,1.6 \mathrm{~Hz}, 3 \mathrm{H}), 6.16(\mathrm{dd}, J=14.4,7.3,1 \mathrm{H}), 3.27-3.03(\mathrm{~m}, 2 \mathrm{H})$, 2.21-1.99 (m, 2H), $1.90(\mathrm{~d}, J=6.2 \mathrm{~Hz}, 1 \mathrm{H}), 1.73(\mathrm{~d}, J=7.8 \mathrm{~Hz}, 4 \mathrm{H}), 1.43-1.24(\mathrm{~m}, 2 \mathrm{H}), 1.03(\mathrm{dd}, J=25.0$, $12.6 \mathrm{~Hz}, 1 \mathrm{H}), 0.94(\mathrm{dd}, J=8.3,5.2 \mathrm{~Hz}, 4 \mathrm{H}), 0.85-0.78(\mathrm{~m}, 3 \mathrm{H}), 0.42-0.35(\mathrm{~m}, 3 \mathrm{H}) .{ }^{13} \mathrm{C}-\mathrm{NMR}(101 \mathrm{MHz}$, $\left.\mathrm{CDCl}_{3}\right) \delta=137.67(\mathrm{~d}, J=2.3 \mathrm{~Hz}), 135.81(\mathrm{~d}, J=12.8 \mathrm{~Hz}), 134.54(\mathrm{~s}), 133.65(\mathrm{~s}), 131.21(\mathrm{~d}, J=2.7 \mathrm{~Hz})$, $130.32(\mathrm{~d}, J=8.7 \mathrm{~Hz}), 128.65(\mathrm{~s}), 128.33(\mathrm{~s}), 128.21(\mathrm{~d}, J=1.5 \mathrm{~Hz}), 126.46(\mathrm{~d}, J=1.2 \mathrm{~Hz}), 117.91(\mathrm{~d}$, $J=7.2 \mathrm{~Hz}), 43.32(\mathrm{~d}, J=3.5 \mathrm{~Hz}), 41.06(\mathrm{~d}, J=66.1 \mathrm{~Hz}), 35.26(\mathrm{~d}, J=2.4 \mathrm{~Hz}), 34.20(\mathrm{~d}, J=1.2 \mathrm{~Hz}), 33.23$ $(\mathrm{d}, J=13.2 \mathrm{~Hz}), 31.19(\mathrm{~d}, J=62.9 \mathrm{~Hz}), 28.31(\mathrm{~d}, J=2.8 \mathrm{~Hz}), 24.61(\mathrm{~d}, J=12.2 \mathrm{~Hz}), 22.59(\mathrm{~s}), 21.53(\mathrm{~s})$, 15.20 (s). HRMS (ESI ${ }^{+}$) Calcd. for $\mathrm{C}_{25} \mathrm{H}_{32} \mathrm{ClOP}\left[\mathrm{M}+\mathrm{Na}^{+}\right.$]: 437.1777, Found: 437.1773.

\subsubsection{The Preparation of $\alpha$-Chlorovinylphosphinate $4 d^{\prime}$}

Dimenthyl 1-chloro-3-phenylprop-1-en-1-ylphosphonate, 4d'

To a suspension of $1 \mathrm{~d}$ ( $38 \mathrm{mg}, 0.075 \mathrm{mmol})$, cuprous iodide $(0.8 \mathrm{mg}, 0.004 \mathrm{mmol}, 5 \mathrm{~mol} \%)$, 1,10-phenanthroline hydrate ( $14 \mathrm{mg}, 0.008 \mathrm{mmol}, 10 \mathrm{~mol} \%)$, and cesium carbonate $(29 \mathrm{mg}, 0.09 \mathrm{mmol})$ in DMF $(0.2 \mathrm{~mL})$, 3a $(7.1 \mu \mathrm{L}, 0.075 \mathrm{mmol})$ was added. The mixture was heated at $80{ }^{\circ} \mathrm{C}$ for $16 \mathrm{~h}$. A saturated aqueous solution of ammonium chloride $(3 \mathrm{~mL})$ was added and the mixture was extracted with dichloromethane $(3 \times 5 \mathrm{~mL})$. The combined organic layer was washed with water $(3 \times 3 \mathrm{~mL})$, dried over anhydrous magnesium sulfate, and concentrated in vacuo. The crude product was purified with preparative TLC with $R_{f}=0.50$ (silica gel, petroleum ether/ethyl acetate $=3: 1$ ). Compound $4 d^{\prime}$ was obtained as a yellow oil. Yield $65 \%(25 \mathrm{mg}),{ }^{31} \mathrm{P}-\mathrm{NMR}\left(162 \mathrm{MHz}, \mathrm{CDCl}_{3}\right) \delta=16.31$ (s). ${ }^{1} \mathrm{H}-\mathrm{NMR}$ $\left(400 \mathrm{MHz}, \mathrm{CDCl}_{3}\right) \delta=7.41(\mathrm{t}, J=7.7 \mathrm{~Hz}, 4 \mathrm{H}), 7.17(\mathrm{~d}, J=7.7 \mathrm{~Hz}, 2 \mathrm{H}), 7.09(\mathrm{dd}, J=21.1 \mathrm{~Hz}, 17.1,1 \mathrm{H})$, $5.46-5.31(\mathrm{~m}, 1 \mathrm{H}), 4.12-3.94(\mathrm{~m}, 2 \mathrm{H}), 2.11(\mathrm{~d}, J=11.8 \mathrm{~Hz}, 1 \mathrm{H}), 1.98(\mathrm{dd}, J=17.3,10.0 \mathrm{~Hz}, 2 \mathrm{H}), 1.85-1.76$ $(\mathrm{m}, 1 \mathrm{H}), 1.37(\mathrm{~s}, 3 \mathrm{H}), 1.17(\mathrm{dd}, J=22.9,12.1 \mathrm{~Hz}, 3 \mathrm{H}), 1.03(\mathrm{dd}, J=23.3,12.0 \mathrm{~Hz}, 2 \mathrm{H}), 0.93(\mathrm{~s}, 2 \mathrm{H})$, $0.91-0.85(\mathrm{~m}, 10 \mathrm{H}), 0.83(\mathrm{dd}, J=6.9,2.6 \mathrm{~Hz}, 7 \mathrm{H}), 0.78(\mathrm{~d}, J=12.5 \mathrm{~Hz}, 1 \mathrm{H}), 0.70(\mathrm{~d}, J=6.9 \mathrm{~Hz}, 3 \mathrm{H}), 0.59$ $(\mathrm{d}, J=6.9 \mathrm{~Hz}, 3 \mathrm{H}) .{ }^{13} \mathrm{C}-\mathrm{NMR}\left(101 \mathrm{MHz}, \mathrm{CDCl}_{3}\right) \delta=130.2(\mathrm{~s}), 128.5(\mathrm{~s}), 77.2(\mathrm{~d}, J=1.9 \mathrm{~Hz}), 48.3(\mathrm{dd}$, $J=6.8,2.6 \mathrm{~Hz}), 43.3(\mathrm{~d}, J=59.4 \mathrm{~Hz}), 34.0(\mathrm{~s}), 31.5(\mathrm{~d}, J=3.8 \mathrm{~Hz}), 25.4(\mathrm{~d}, J=11.9 \mathrm{~Hz}), 22.8(\mathrm{~d}, J=7.0 \mathrm{~Hz})$, $21.9(\mathrm{~d}, \mathrm{~J}=7.8 \mathrm{~Hz}), 21.0(\mathrm{~s}), 15.7\left(\mathrm{~d}, \mathrm{~J}=12.8 \mathrm{~Hz}\right.$ ). HRMS (ESI ${ }^{+}$) Calcd. for $\mathrm{C}_{29} \mathrm{H}_{49} \mathrm{ClO}_{3} \mathrm{P}\left[\mathrm{M}+\mathrm{H}^{+}\right]$: 509.2951, Found: 509.2954 . 


\subsubsection{Preparation of $\gamma$-Keto Phosphorus Derivatives 5}

$\left(S_{P}\right)$-Menthyl 3-oxo-3-phenylpropyl phenylphosphinate, 5a

To a solution of $1 \mathrm{a}(50 \mathrm{mg}, 0.116 \mathrm{mmol})$, palladium acetate $(1.3 \mathrm{mg}, 0.058 \mathrm{mmol}, 5 \mathrm{~mol} \%)$, and triphenyl phosphine $(6.1 \mathrm{mg}, 0.232 \mathrm{mmol}, 20 \mathrm{~mol} \%)$ in toluene $(0.5 \mathrm{~mL})$, 3a $(25 \mu \mathrm{L}, 0.255 \mathrm{mmol})$ was added. The mixture was heated at $120^{\circ} \mathrm{C}$ for $24 \mathrm{~h}$ and monitored with TLC. After removing the solvent in vacuo, the residue was dissolved in dichloromethane, filtered over silica gel, and washed with dichloromethane. After removing the solvent in vacuo, the residue was purified with preparative TLC with $R_{f}=0.55$ (silica gel, petroleum ether/ethyl acetate $=1 / 1$ as eluent). The pure $5 \mathbf{a}$ was obtained as a yellow solid. Yield $89 \%$ (43 mg), m.p. 95.6-100.7 ${ }^{\circ} \mathrm{C} .{ }^{31} \mathrm{P}-\mathrm{NMR}\left(162 \mathrm{MHz}, \mathrm{CDCl}_{3}\right) \delta=41.90(\mathrm{~s})$. ${ }^{1} \mathrm{H}-\mathrm{NMR}\left(400 \mathrm{MHz}, \mathrm{CDCl}_{3}\right) \delta=7.91(\mathrm{~d}, J=7.8 \mathrm{~Hz}, 2 \mathrm{H}), 7.84(\mathrm{dd}, J=11.6,7.4 \mathrm{~Hz}, 2 \mathrm{H}), 7.55(\mathrm{~d}, J=7.6 \mathrm{~Hz}$, $2 \mathrm{H}), 7.51-7.42(\mathrm{~m}, 4 \mathrm{H}), 4.36-4.28(\mathrm{~m}, 1 \mathrm{H}), 3.33(\mathrm{~s}, 1 \mathrm{H}), 3.11(\mathrm{~s}, 1 \mathrm{H}), 2.45-2.30(\mathrm{~m}, 1 \mathrm{H}), 2.30-2.20(\mathrm{~m}, 2 \mathrm{H})$, $1.74(\mathrm{~d}, J=11.5 \mathrm{~Hz}, 1 \mathrm{H}), 1.63(\mathrm{~d}, J=27.1 \mathrm{~Hz}, 4 \mathrm{H}), 1.44-1.34(\mathrm{~m}, 1 \mathrm{H}), 1.26(\mathrm{~s}, 1 \mathrm{H}), 1.04-0.97(\mathrm{~m}, 1 \mathrm{H}), 0.91$ $(\mathrm{dd}, J=14.0,7.0 \mathrm{~Hz}, 7 \mathrm{H}), 0.80(\mathrm{~d}, J=12.3 \mathrm{~Hz}, 1 \mathrm{H}), 0.74(\mathrm{~d}, J=6.6 \mathrm{~Hz}, 3 \mathrm{H}) .{ }^{13} \mathrm{C}-\mathrm{NMR}\left(101 \mathrm{MHz}, \mathrm{CDCl}_{3}\right)$ $\delta=197.7(\mathrm{~d}, J=15.7 \mathrm{~Hz}), 136.2(\mathrm{~s}), 133.3(\mathrm{~s}), 133.1(\mathrm{~s}), 132.1(\mathrm{~s}), 131.9(\mathrm{~s}), 131.3(\mathrm{~d}, J=9.9 \mathrm{~Hz}), 128.6(\mathrm{~s})$, $128.6(\mathrm{~s}), 128.4(\mathrm{~s}), 128.0(\mathrm{~s}), 76.6(\mathrm{~s}), 48.7(\mathrm{~d}, J=6.0 \mathrm{~Hz}), 43.1(\mathrm{~s}), 34.0(\mathrm{~s}), 31.4(\mathrm{~s}), 30.9(\mathrm{~s}), 25.7(\mathrm{~s}), 24.4(\mathrm{~d}$, $J=103.3 \mathrm{~Hz}$ ), $22.8(\mathrm{~s}), 21.9$ (s), 21.1 (s), $15.7(\mathrm{~s}) . \mathrm{HRMS}\left(\mathrm{ESI}^{+}\right)$Calcd. for $\mathrm{C}_{25} \mathrm{H}_{33} \mathrm{O}_{3} \mathrm{P}\left[\mathrm{M}+\mathrm{H}^{+}\right]: 413.2246$, Found: 413.2244 .

\section{Ethyl 3-oxo-3-phenylpropyl phenylphosphinate, 5b}

The compound $\mathbf{5 b}$ was obtained as a yellow solid. Yield $81 \%$ (38 $\mathrm{mg}$, containing $79 \%$ of $5 \mathrm{~b}$ and ca. $21 \%$ of triphenyl phosphine oxide). $R_{f}=0.55$ (petroleum ether $/$ ethyl acetate $\left.=1: 1\right) .{ }^{31} \mathrm{P}-\mathrm{NMR}(162$ $\left.\mathrm{MHz}, \mathrm{CDCl}_{3}\right) \delta=44.36(\mathrm{~s}, 81 \%), 29.14(\mathrm{~s}, 19 \%) .{ }^{1} \mathrm{H}-\mathrm{NMR}\left(400 \mathrm{MHz}, \mathrm{CDCl}_{3}\right) \delta=7.93-7.88(\mathrm{~m}, 2 \mathrm{H})$, 7.83-7.76 (m, 2H), 7.68-7.61 (m, 2H), 7.56-7.44 (m, 6H), 7.44-7.39 (m, 3H), 4.08 (dd, J = 10.1, 7.1 Hz, $1 \mathrm{H}), 3.85(\mathrm{dd}, J=10.1,7.1 \mathrm{~Hz}, 1 \mathrm{H}), 3.34(\mathrm{dddd}, J=18.1,11.0,9.1,5.1 \mathrm{~Hz}, 1 \mathrm{H}), 3.16(\mathrm{dddd}, J=18.1,10.8$, 9.6, $4.9 \mathrm{~Hz}, 1 \mathrm{H}), 2.44-2.20(\mathrm{~m}, 3 \mathrm{H}), 1.26(\mathrm{t}, J=7.1 \mathrm{~Hz}, 3 \mathrm{H})$.

\section{Diethyl 3-oxo-3-phenyl propylphosphonate, 5c}

The pure $5 \mathrm{c}$ was obtained as a yellow solid. Yield $83 \%(39 \mathrm{mg}) R_{f}=0.63$ (petroleum ether/ethyl acetate $=1: 1)$, m.p. $78.5-83.6^{\circ} \mathrm{C} .{ }^{31} \mathrm{P}-\mathrm{NMR}\left(162 \mathrm{MHz}, \mathrm{CDCl}_{3}\right) \delta=31.77(\mathrm{~s}) .{ }^{1} \mathrm{H}-\mathrm{NMR}\left(400 \mathrm{MHz}, \mathrm{CDCl}_{3}\right)$ $\delta=8.01-7.91(\mathrm{~m}, 2 \mathrm{H}), 7.58(\mathrm{t}, J=7.4 \mathrm{~Hz}, 1 \mathrm{H}), 7.48(\mathrm{t}, J=7.6 \mathrm{~Hz}, 2 \mathrm{H}), 4.19-4.02(\mathrm{~m}, 4 \mathrm{H}), 3.30$ (ddd, $J=10.6,7.9,4.8 \mathrm{~Hz}, 2 \mathrm{H}), 2.19$ (dddd, $J=39.0,37.6,20.4,17.3 \mathrm{~Hz}, 2 \mathrm{H}), 1.37-1.24(\mathrm{~m}, 6 \mathrm{H}) .{ }^{13} \mathrm{C}-\mathrm{NMR}(101$ $\left.\mathrm{MHz}, \mathrm{CDCl}_{3}\right) \delta=197.4(\mathrm{~d}, J=15.7 \mathrm{~Hz}), 136.3(\mathrm{~s}), 133.3(\mathrm{~s}), 128.7(\mathrm{~s}), 128.0(\mathrm{~s}), 61.7(\mathrm{~d}, J=6.5 \mathrm{~Hz}), 39.4$ $(\mathrm{s}), 31.7(\mathrm{~d}, J=3.0 \mathrm{~Hz}), 23.3(\mathrm{~s}), 20.6-18.9(\mathrm{~m}), 16.4(\mathrm{~d}, J=6.0 \mathrm{~Hz}), 13.7(\mathrm{~s})$. HRMS (ESI ${ }^{+}$) Calcd. for $\mathrm{C}_{13} \mathrm{H}_{19} \mathrm{O}_{4} \mathrm{P}[\mathrm{M}]: 271.1099$, Found: 271.1103.

Dimenthyl 3-oxo-3-phenylpropyl phenylphosphonate, 5d

The pure $5 \mathbf{d}$ was obtained as a yellow oil. Yield $89 \%(43 \mathrm{mg}) . R_{f}=0.54$ (petroleum ether/ethyl acetate $=5: 1) .{ }^{31} \mathrm{P}-\mathrm{NMR}\left(400 \mathrm{MHz}, \mathrm{CDCl}_{3}\right) \delta=29.24(\mathrm{~s}) .{ }^{1} \mathrm{H}-\mathrm{NMR}\left(400 \mathrm{MHz}, \mathrm{CDCl}_{3}\right) \delta=7.98(\mathrm{~d}$, $J=7.9 \mathrm{~Hz}, 2 \mathrm{H}), 7.58(\mathrm{t}, J=7.3 \mathrm{~Hz}, 1 \mathrm{H}), 7.48(\mathrm{t}, J=7.3 \mathrm{~Hz}, 2 \mathrm{H}), 4.27-4.18(\mathrm{~m}, 2 \mathrm{H}), 3.29(\mathrm{dd}, J=16.0,8.7$ $\mathrm{Hz}, 2 \mathrm{H}), 2.26(\mathrm{~s}, 2 \mathrm{H}), 2.22-2.05(\mathrm{~m}, 4 \mathrm{H}), 1.66(\mathrm{~d}, J=11.6 \mathrm{~Hz}, 4 \mathrm{H}), 1.45(\mathrm{~s}, 2 \mathrm{H}), 1.31(\mathrm{~s}, 2 \mathrm{H}), 1.20-1.09(\mathrm{~m}$, 2H), $1.01(\mathrm{dd}, J=24.9,13.2 \mathrm{~Hz}, 3 \mathrm{H}), 0.91(\mathrm{~d}, J=4.0 \mathrm{~Hz}, 12 \mathrm{H}), 0.84(\mathrm{dd}, J=14.0,7.7 \mathrm{~Hz}, 7 \mathrm{H}) .{ }^{13} \mathrm{C}-\mathrm{NMR}$ $\left(101 \mathrm{MHz}, \mathrm{CDCl}_{3}\right) \delta=197.8(\mathrm{~d}, J=16.7 \mathrm{~Hz}$ ) $, 136.4(\mathrm{~s}), 133.2(\mathrm{~d}, J=2.8 \mathrm{~Hz}), 128.6(\mathrm{~s}), 128.0(\mathrm{~s}), 77.2$ $(\mathrm{d}, J=7.4 \mathrm{~Hz}), 48.6(\mathrm{~s}), 43.7(\mathrm{~s}), 43.1(\mathrm{~s}), 34.1(\mathrm{~s}), 32.2(\mathrm{~d}, J=3.2 \mathrm{~Hz}), 31.5(\mathrm{~d}, J=6.5 \mathrm{~Hz}), 25.7(\mathrm{~s}), 25.4$ (s), 22.8 (s), 22.5 (s), 21.9 (s), 21.0 (s), 15.8 (s), 15.6 (s). HRMS (ESI ${ }^{+}$) Calcd. for $\mathrm{C}_{29} \mathrm{H}_{47} \mathrm{O}_{4} \mathrm{P}\left[\mathrm{M}+\mathrm{H}^{+}\right]$: 491.3290, Found: 491.3297.

\section{Diethyl 3-oxo-3-p-tolyl propylphosphonate, 5e}

The compound $5 \mathbf{e}$ was obtained as a yellow solid. Yield $88 \%(41 \mathrm{mg}$, containing $90 \%$ of $5 \mathbf{e}$ and ca. $10 \%$ of triphenyl phosphine oxide, $5 \mathbf{e}$ was obtained in a yield of $88 \%$ ). $R_{f}=0.58$ (petroleum ether/ethyl acetate $=1: 1) .{ }^{31} \mathrm{P}-\mathrm{NMR}\left(162 \mathrm{MHz}, \mathrm{CDCl}_{3}\right) \delta=31.90(\mathrm{~s}, 93 \%), 29.13(\mathrm{~s}, 7 \%) .{ }^{1} \mathrm{H}-\mathrm{NMR}\left(400 \mathrm{MHz}, \mathrm{CDCl}_{3}\right)$ 
$\delta=7.86(\mathrm{~d}, J=8.0 \mathrm{~Hz}, 2 \mathrm{H}), 7.66(\mathrm{dd}, J=12.0,7.1 \mathrm{~Hz}, 0.58 \mathrm{H}), 7.58-7.50(\mathrm{~m}, 0.28 \mathrm{H}), 7.46(\mathrm{~d}, J=5.0 \mathrm{~Hz}$, $0.59 \mathrm{H}), 7.25(\mathrm{~d}, J=7.9 \mathrm{~Hz}, 2 \mathrm{H}), 4.19-4.02(\mathrm{~m}, 4 \mathrm{H}), 3.29-3.18(\mathrm{~m}, 2 \mathrm{H}), 2.40(\mathrm{~s}, 3 \mathrm{H}), 2.22-2.10(\mathrm{~m}, 2 \mathrm{H}), 1.32$ $(\mathrm{t}, J=7.1 \mathrm{~Hz}, 6 \mathrm{H})$.

Diphenyl 3-oxo-3-phenyl propylphosphine oxide, $\mathbf{5 f}$

The pure $\mathbf{5 f}$ was obtained as a yellow solid. Yield $87 \%(41 \mathrm{mg}) . R_{f}=0.48$ (petroleum ether/ethyl acetate $=1: 1)$, m.p. $94.3-99.1{ }^{\circ} \mathrm{C} .{ }^{31} \mathrm{P}-\mathrm{NMR}\left(162 \mathrm{MHz}, \mathrm{CDCl}_{3}\right) \delta=28.03$ (s). ${ }^{1} \mathrm{H}-\mathrm{NMR}\left(400 \mathrm{MHz}, \mathrm{CDCl}_{3}\right)$ $\delta=7.92(\mathrm{~d}, J=8.3 \mathrm{~Hz}, 2 \mathrm{H}), 7.79(\mathrm{dd}, J=11.4,8.1 \mathrm{~Hz}, 4 \mathrm{H}), 7.71-7.34(\mathrm{~m}, 9 \mathrm{H}), 3.33(\mathrm{dd}, J=15.8,7.9 \mathrm{~Hz}$, 2H), $2.74(\mathrm{dd}, J=16.1,10.4 \mathrm{~Hz}, 2 \mathrm{H}) .{ }^{13} \mathrm{C}-\mathrm{NMR}\left(101 \mathrm{MHz}, \mathrm{CDCl}_{3}\right) \delta=197.8(\mathrm{~d}, J=14.1 \mathrm{~Hz}), 136.2(\mathrm{~s})$, $133.4(\mathrm{~s}), 133.0(\mathrm{~s}), 131.9(\mathrm{~d}, J=2.7 \mathrm{~Hz}), 130.8(\mathrm{~d}, J=9.3 \mathrm{~Hz}), 128.8(\mathrm{~s}), 128.7(\mathrm{~s}), 128.6(\mathrm{~s}), 128.1(\mathrm{~s}), 30.7$ (s), $23.7\left(\mathrm{~d}, J=73.9 \mathrm{~Hz}\right.$ ). HRMS (ESI ${ }^{+}$) Calcd. for $\mathrm{C}_{21} \mathrm{H}_{19} \mathrm{O}_{2} \mathrm{P}$ [M]: 335.1201, Found: 335.1201.

$\left(S_{P}\right)$-Menthyl 3-oxo-3-phenylpropyl phenylphosphine oxide, $\mathbf{5 g} / \mathbf{5} \mathbf{g}^{\prime}$

Compound $5 \mathrm{~g} / 5 \mathbf{g}^{\prime}$ was obtained as a yellow solid. Yield $85 \%(40 \mathrm{mg}) . R_{f}=0.43$ (petroleum ether/ethyl acetate $=1: 1)$, m.p. $96.5-100.8{ }^{\circ} \mathrm{C} .{ }^{31} \mathrm{P}-\mathrm{NMR}\left(162 \mathrm{MHz}, \mathrm{CDCl}_{3}\right) \delta=45.87(\mathrm{~s}, 20 \%), 43.78$ (s, 80\%). ${ }^{1} \mathrm{H}-\mathrm{NMR}\left(400 \mathrm{MHz}, \mathrm{CDCl}_{3}\right) \delta=7.88(\mathrm{dd}, J=17.0,7.7 \mathrm{~Hz}, 2 \mathrm{H}), 7.79-7.68(\mathrm{~m}, 2 \mathrm{H}), 7.62-7.44$ $(\mathrm{m}, 4 \mathrm{H}), 7.40(\mathrm{t}, J=7.6 \mathrm{~Hz}, 2 \mathrm{H}), 3.42(\mathrm{ddd}, J=18.2,11.6,6.2 \mathrm{~Hz}, 1 \mathrm{H}), 3.00-2.43(\mathrm{~m}, 1 \mathrm{H}), 2.33-2.14(\mathrm{~m}$, $2 \mathrm{H}), 2.11-1.88(\mathrm{~m}, 3 \mathrm{H}), 1.73(\mathrm{~d}, J=8.8 \mathrm{~Hz}, 3 \mathrm{H}), 1.46-1.19(\mathrm{~m}, 3 \mathrm{H}), 1.03(\mathrm{dd}, J=29.4,16.0 \mathrm{~Hz}, 1 \mathrm{H}), 0.92$ $(\mathrm{d}, J=6.3 \mathrm{~Hz}, 3 \mathrm{H}), 0.86(\mathrm{t}, J=6.3 \mathrm{~Hz}, 4 \mathrm{H}), 0.79(\mathrm{~d}, J=6.9 \mathrm{~Hz}, 1 \mathrm{H}), 0.39(\mathrm{~d}, J=6.7 \mathrm{~Hz}, 2 \mathrm{H}) .{ }^{13} \mathrm{C}-\mathrm{NMR}$ $\left(101 \mathrm{MHz}, \mathrm{CDCl}_{3}\right) \delta=198.4(\mathrm{~d}, J=13.0 \mathrm{~Hz}), 133.7(\mathrm{~d}, J=93.4 \mathrm{~Hz}), 131.6-130.9(\mathrm{~m}), 130.6(\mathrm{~d}, J=8.6$ $\mathrm{Hz}), 128.5(\mathrm{t}, J=5.4 \mathrm{~Hz}), 128.0(\mathrm{~d}, J=4.4 \mathrm{~Hz}), 43.4(\mathrm{~d}, J=3.3 \mathrm{~Hz}), 41.5(\mathrm{dd}, J=67.3,51.8 \mathrm{~Hz}), 35.8(\mathrm{~d}$, $J=78.0 \mathrm{~Hz}), 34.2(\mathrm{~s}), 33.2(\mathrm{dd}, J=13.2,7.4 \mathrm{~Hz}), 30.6(\mathrm{~s}), 28.4(\mathrm{~d}, J=19.9 \mathrm{~Hz}), 24.6(\mathrm{~d}, J=12.3 \mathrm{~Hz}), 22.2$ $(\mathrm{d}, J=57.4 \mathrm{~Hz}), 21.5-21.2(\mathrm{~m}), 15.4(\mathrm{~d}, J=55.2 \mathrm{~Hz})$. HRMS (ESI ${ }^{+}$) Calcd. for $\mathrm{C}_{25} \mathrm{H}_{33} \mathrm{O}_{2} \mathrm{P}[\mathrm{M}]: 397.2296$, Found: 397.2303.

\subsubsection{Preparation of $\gamma$-Amino Phosphorous Derivatives 11}

$\left(S_{P}\right)$-Menthyl-3-butylamino-3-phenylpropyl phenylphosphinate, 11aa

To a solution of 1a (50 mg, $0.116 \mathrm{mmol})$, palladium acetate $(1.3 \mathrm{mg}, 0.058 \mathrm{mmol}, 5 \mathrm{~mol} \%)$, and triphenyl phosphine $(6.1 \mathrm{mg}, 0.232 \mathrm{mmol}, 20 \mathrm{~mol} \%)$ in toluene $(0.5 \mathrm{~mL}), 3 \mathbf{a}(22 \mu \mathrm{L}, 0.225 \mathrm{mmol})$ was added. The mixture was heated at $120^{\circ} \mathrm{C}$ for $24 \mathrm{~h}$, monitored with TLC, and then cooled to room temperature. Ethanol $(2 \mathrm{~mL})$ and sodium borohydride $(8.7 \mathrm{mg}, 0.232 \mathrm{mmol})$ were added. The mixture was stirred at room temperature for $10 \mathrm{~h}$. After a saturated solution of ammonium chloride $(3 \mathrm{~mL})$ was added, the mixture was extracted with dichloromethane $(3 \times 5 \mathrm{~mL})$ and washed with water $(3 \times 3 \mathrm{~mL})$. The residue was purified with preparative TLC with $R_{f}=0.40$ (silica gel, methanol/dichloromethane $=$ $1 / 20$ as eluent). The pure 11aa was obtained as a yellow oil. Yield $87 \%(47 \mathrm{mg}) .{ }^{31} \mathrm{P}-\mathrm{NMR}(162 \mathrm{MHz}$, $\left.\mathrm{CDCl}_{3}\right) \delta=37.39$ (s). ${ }^{1} \mathrm{H}-\mathrm{NMR}\left(400 \mathrm{MHz}, \mathrm{CDCl}_{3}\right) \delta=7.77-7.65(\mathrm{~m}, 2 \mathrm{H}), 7.52(\mathrm{t}, J=7.3 \mathrm{~Hz}, 1 \mathrm{H}), 7.49-7.39$ $(\mathrm{m}, 2 \mathrm{H}), 7.29(\mathrm{dd}, J=12.3,5.9 \mathrm{~Hz}, 5 \mathrm{H}), 4.27-4.09(\mathrm{~m}, 1 \mathrm{H}), 3.77(\mathrm{~d}, J=33.2 \mathrm{~Hz}, 1 \mathrm{H}), 2.46(\mathrm{~d}, J=7.1 \mathrm{~Hz}$, $2 \mathrm{H}), 2.23-1.93(\mathrm{~m}, 3 \mathrm{H}), 1.84(\mathrm{dt}, J=36.0,14.3 \mathrm{~Hz}, 2 \mathrm{H}), 1.74-1.55(\mathrm{~m}, 4 \mathrm{H}), 1.55-1.44(\mathrm{~m}, 2 \mathrm{H}), 1.38-1.18$ $(\mathrm{m}, 4 \mathrm{H}), 1.03-0.88(\mathrm{~m}, 5 \mathrm{H}), 0.82(\mathrm{ddd}, J=12.1,7.0,3.6 \mathrm{~Hz}, 6 \mathrm{H}), 0.77-0.68(\mathrm{~m}, 3 \mathrm{H}) .{ }^{13} \mathrm{C}-\mathrm{NMR}(101$ $\left.\mathrm{MHz}, \mathrm{CDCl}_{3}\right) \delta=143.1(\mathrm{~s}), 133.5(\mathrm{~d}, J=22.0 \mathrm{~Hz}), 132.3(\mathrm{~d}, J=21.5 \mathrm{~Hz}), 131.8(\mathrm{~d}, J=2.6 \mathrm{~Hz}), 131.4(\mathrm{~d}$, $J=2.2 \mathrm{~Hz}), 131.3(\mathrm{~d}, J=2.2 \mathrm{~Hz}), 128.5-128.3(\mathrm{~m}), 128.2(\mathrm{~s}), 127.2(\mathrm{t}, J=7.1 \mathrm{~Hz}), 76.4-76.1(\mathrm{~m}), 64.0-63.2$ $(\mathrm{m}), 48.8(\mathrm{~d}, J=4.4 \mathrm{~Hz}), 47.3(\mathrm{~d}, J=4.3 \mathrm{~Hz}), 43.2(\mathrm{~s}), 34.1(\mathrm{~s}), 32.3(\mathrm{~s}), 31.4(\mathrm{~s}), 29.7(\mathrm{~s}), 27.0(\mathrm{dd}, J=100.8$, $10.1 \mathrm{~Hz}), 25.6(\mathrm{~s}), 22.8(\mathrm{~s}), 21.9(\mathrm{~s}), 21.1(\mathrm{~s}), 20.4(\mathrm{~s}), 15.7(\mathrm{~d}, J=2.6 \mathrm{~Hz}), 13.9(\mathrm{~s})$. HRMS (ESI ${ }^{+}$) Calcd. for $\mathrm{C}_{29} \mathrm{H}_{44} \mathrm{NO}_{2} \mathrm{P}[\mathrm{M}]: 470.3188$, Found: 470.3188 .

$\left(S_{P}\right)$-Menthyl-3-phenethylamino-3-phenylpropyl phenylphosphinate, 11ab

The pure 11ab was obtained as a yellow oil. Yield 83\% (35 mg). $\quad R_{f}=0.46$ (methanol/dichloromethane = 1:20). ${ }^{31} \mathrm{P}-\mathrm{NMR}\left(162 \mathrm{MHz}, \mathrm{CDCl}_{3}\right) \delta=43.35$ (s, 48\%), $43.20(\mathrm{~s}, 52 \%)$. ${ }^{1} \mathrm{H}-\mathrm{NMR}\left(400 \mathrm{MHz}, \mathrm{CDCl}_{3}\right) \delta=7.69(\mathrm{dd}, J=19.5,10.4 \mathrm{~Hz}, 2 \mathrm{H}), 7.57-7.38(\mathrm{~m}, 4 \mathrm{H}), 7.29(\mathrm{~s}, 2 \mathrm{H}), 7.24(\mathrm{~d}$, $J=7.4 \mathrm{~Hz}, 2 \mathrm{H}), 7.15(\mathrm{dd}, J=22.6,10.8 \mathrm{~Hz}, 5 \mathrm{H}), 4.01-3.86(\mathrm{~m}, 1 \mathrm{H}), 3.59(\mathrm{~s}, 1 \mathrm{H}), 2.80-2.59(\mathrm{~m}, 4 \mathrm{H}), 2.30(\mathrm{~s}$, 
$1 \mathrm{H}), 2.00-1.78(\mathrm{~m}, 5 \mathrm{H}), 1.60(\mathrm{~s}, 4 \mathrm{H}), 1.31-1.10(\mathrm{~m}, 3 \mathrm{H}), 0.84(\mathrm{dd}, J=37.1,6.5 \mathrm{~Hz}, 8 \mathrm{H}), 0.36-0.21(\mathrm{~m}, 3 \mathrm{H})$. ${ }^{13} \mathrm{C}-\mathrm{NMR}\left(101 \mathrm{MHz}, \mathrm{CDCl}_{3}\right) \delta=142.8(\mathrm{~s}), 139.9(\mathrm{~s}), 131.9(\mathrm{~s}), 131.4(\mathrm{dd}, J=9.7,5.2 \mathrm{~Hz}), 130.7(\mathrm{~d}, J=11.8$ $\mathrm{Hz}), 128.6(\mathrm{~s}), 128.4(\mathrm{~d}, J=1.6 \mathrm{~Hz}), 128.4(\mathrm{~d}, J=3.6 \mathrm{~Hz}), 128.3(\mathrm{~s}), 127.3(\mathrm{~d}, J=3.0 \mathrm{~Hz}), 127.2(\mathrm{~s}), 126.1$ (s), $76.6(\mathrm{~d}, J=2.4 \mathrm{~Hz}), 63.4(\mathrm{dd}, J=16.4,9.0 \mathrm{~Hz}), 48.7(\mathrm{~d}, J=6.7 \mathrm{~Hz}), 48.6(\mathrm{~d}, J=3.6 \mathrm{~Hz}), 43.9(\mathrm{~s}), 36.3$ (s), $34.1(\mathrm{~s}), 31.5(\mathrm{~s}), 29.6(\mathrm{~s}), 26.5(\mathrm{~d}, J=101.8 \mathrm{~Hz}), 25.3(\mathrm{~s}), 22.6(\mathrm{~s}), 22.0(\mathrm{~s}), 21.0(\mathrm{~s}), 15.1(\mathrm{~d}, J=4.6 \mathrm{~Hz})$. HRMS $\left(\right.$ ESI $^{+}$) Calcd. for $\mathrm{C}_{33} \mathrm{H}_{44} \mathrm{NO}_{2} \mathrm{P}$ [M]: 518.3188, Found: 518.3185 .

$\left(S_{P}\right)$-Menthyl 3-phenyl-3-pyrrolidin-1-yl propylphosphinate, 11ac

The pure 11ac was obtained as a yellow oil. Yield $83 \%(35 \mathrm{mg}) . \quad R_{f}=0.40$ (methanol/dichloromethane = 1:20). ${ }^{31} \mathrm{P}-\mathrm{NMR}\left(162 \mathrm{MHz}, \mathrm{CDCl}_{3}\right) \delta=42.71(\mathrm{~s}, 51 \%), 42.50(\mathrm{~s}, 49 \%)$. ${ }^{1} \mathrm{H}-\mathrm{NMR}\left(400 \mathrm{MHz}, \mathrm{CDCl}_{3}\right) \delta=7.65(\mathrm{dd}, J=18.9,10.6 \mathrm{~Hz}, 2 \mathrm{H}), 7.48(\mathrm{~s}, 1 \mathrm{H}), 7.40(\mathrm{~s}, 2 \mathrm{H}), 7.29-7.19$ $(\mathrm{m}, 3 \mathrm{H}), 7.16(\mathrm{t}, J=7.1 \mathrm{~Hz}, 2 \mathrm{H}), 4.28-4.13(\mathrm{~m}, 1 \mathrm{H}), 3.04(\mathrm{~d}, J=5.5 \mathrm{~Hz}, 1 \mathrm{H}), 2.45(\mathrm{~s}, 2 \mathrm{H}), 2.27(\mathrm{~s}, 2 \mathrm{H})$, 2.20-2.04 (m, 2H), $1.97(\mathrm{~s}, 2 \mathrm{H}), 1.77-1.43(\mathrm{~m}, 9 \mathrm{H}), 1.30(\mathrm{dd}, J=26.6,13.7 \mathrm{~Hz}, 2 \mathrm{H}), 1.03-0.84(\mathrm{~m}, 5 \mathrm{H})$, $0.80(\mathrm{t}, J=7.7 \mathrm{~Hz}, 3 \mathrm{H}), 0.70(\mathrm{t}, J=5.4 \mathrm{~Hz}, 3 \mathrm{H}) .{ }^{13} \mathrm{C}-\mathrm{NMR}\left(101 \mathrm{MHz}, \mathrm{CDCl}_{3}\right) \delta=141.9(\mathrm{~d}, J=19.1 \mathrm{~Hz})$, $132.9(\mathrm{dd}, J=124.2,57.6 \mathrm{~Hz}), 131.9-131.7(\mathrm{~m}), 131.3(\mathrm{dd}, J=12.2,9.8 \mathrm{~Hz}), 128.3(\mathrm{~s}), 128.2(\mathrm{~s}), 128.2(\mathrm{~s})$, $128.1(\mathrm{~s}), 127.1(\mathrm{~d}, J=7.5 \mathrm{~Hz}), 76.2(\mathrm{dd}, J=18.1,7.4 \mathrm{~Hz}), 70.9(\mathrm{dd}, J=37.9,17.2 \mathrm{~Hz}), 52.7(\mathrm{~s}), 52.4(\mathrm{~s})$, $48.9-48.6(\mathrm{~m}), 43.1(\mathrm{~d}, J=7.7 \mathrm{~Hz}), 34.0(\mathrm{~s}), 31.4(\mathrm{~d}, J=1.7 \mathrm{~Hz}), 27.6(\mathrm{~d}, J=4.7 \mathrm{~Hz}), 26.5(\mathrm{dd}, J=101.2$, $19.3 \mathrm{~Hz}), 25.6(\mathrm{~s}), 23.2(\mathrm{~d}, J=2.5 \mathrm{~Hz}), 22.8(\mathrm{~s}), 21.9(\mathrm{~s}), 21.1(\mathrm{~s}), 15.6(\mathrm{~d}, J=3.8 \mathrm{~Hz})$. HRMS (ESI ${ }^{+}$) Calcd. for $\mathrm{C}_{29} \mathrm{H}_{42} \mathrm{NO}_{2} \mathrm{P}\left[\mathrm{M}+\mathrm{H}^{+}\right]$: 468.3031, Found: 468.3027 .

\section{Ethyl 3-butylamino-3-phenylpropyl phenylphosphinate, 11ba}

The pure 11ba was obtained as a yellow oil. Yield $90 \%$ (50 mg). $R_{f}=0.41$ (ethyl acetate). ${ }^{31} \mathrm{P}-\mathrm{NMR}$ $\left(162 \mathrm{MHz}, \mathrm{CDCl}_{3}\right) \delta=44.85$ (s). ${ }^{1} \mathrm{H}-\mathrm{NMR}\left(400 \mathrm{MHz}, \mathrm{CDCl}_{3}\right) \delta=7.70(\mathrm{ddd}, J=11.6,9.2,6.8 \mathrm{~Hz}, 2 \mathrm{H})$, $7.57-7.49(\mathrm{~m}, 1 \mathrm{H}), 7.49-7.37(\mathrm{~m}, 2 \mathrm{H}), 7.30(\mathrm{~d}, J=7.0 \mathrm{~Hz}, 1 \mathrm{H}), 7.25-7.21(\mathrm{~m}, 1 \mathrm{H}), 7.18(\mathrm{~d}, J=7.2 \mathrm{~Hz}$, $2 \mathrm{H}), 4.03(\mathrm{dt}, J=20.1,6.4 \mathrm{~Hz}, 1 \mathrm{H}), 3.80(\mathrm{dt}, J=12.4,7.3 \mathrm{~Hz}, 1 \mathrm{H}), 3.55(\mathrm{dd}, J=12.8,6.5 \mathrm{~Hz}, 1 \mathrm{H}), 2.37$ $(\mathrm{dt}, J=6.9,5.1 \mathrm{~Hz}, 2 \mathrm{H}), 2.05-1.63(\mathrm{~m}, 5 \mathrm{H}), 1.52(\mathrm{~s}, 1 \mathrm{H}), 1.36(\mathrm{~d}, J=4.2 \mathrm{~Hz}, 2 \mathrm{H}), 1.25(\mathrm{td}, J=7.0,3.8 \mathrm{~Hz}$, $5 \mathrm{H}), 0.84(\mathrm{t}, J=7.3 \mathrm{~Hz}, 3 \mathrm{H}) .{ }^{13} \mathrm{C}-\mathrm{NMR}\left(101 \mathrm{MHz}, \mathrm{CDCl}_{3}\right) \delta=143.1(\mathrm{~s}), 132.1(\mathrm{~d}, J=2.7 \mathrm{~Hz}), 131.6(\mathrm{~s})$, $131.5(\mathrm{~s}), 130.2(\mathrm{~d}, J=15.7 \mathrm{~Hz}), 128.6(\mathrm{~s}), 128.5(\mathrm{~s}), 128.4(\mathrm{~s}), 127.5-127.0(\mathrm{~m}), 63.6(\mathrm{~d}, J=18.8 \mathrm{~Hz}), 60.5(\mathrm{~d}$, $J=6.2 \mathrm{~Hz}), 47.3(\mathrm{~d}, J=3.8 \mathrm{~Hz}), 32.3(\mathrm{~s}), 29.6(\mathrm{~d}, J=2.8 \mathrm{~Hz}), 26.3(\mathrm{dd}, J=101.3,21.0 \mathrm{~Hz}), 20.4(\mathrm{~s}), 16.4(\mathrm{~d}$, $J=6.5 \mathrm{~Hz}$ ), 13.9 (s). HRMS (ESI ${ }^{+}$) Calcd. for $\mathrm{C}_{21} \mathrm{H}_{30} \mathrm{NO}_{2} \mathrm{P}$ [M]: 360.2092, Found: 360.2094 .

Ethyl 3-phenethylamino-3-phenylpropyl phenylphosphinate, $\mathbf{1 1 b b}$

The pure $\mathbf{1 1 b b}$ was obtained as a yellow oil. Yield $85 \%$ (54 mg). $R_{f}=0.32$ (ethyl acetate). ${ }^{31} \mathrm{P}-\mathrm{NMR}$ $\left(162 \mathrm{MHz}, \mathrm{CDCl}_{3}\right) \delta=44.76(\mathrm{~s}) .{ }^{1} \mathrm{H}-\mathrm{NMR}\left(400 \mathrm{MHz}, \mathrm{CDCl}_{3}\right) \delta=7.68(\mathrm{ddd}, J=11.3,9.9,4.3 \mathrm{~Hz}, 2 \mathrm{H}), 7.53$ $(\mathrm{t}, J=6.8 \mathrm{~Hz}, 1 \mathrm{H}), 7.44(\mathrm{dd}, J=8.9,5.8 \mathrm{~Hz}, 2 \mathrm{H}), 7.26(\mathrm{dt}, J=10.8,7.3 \mathrm{~Hz}, 5 \mathrm{H}), 7.18(\mathrm{~d}, J=7.3 \mathrm{~Hz}, 1 \mathrm{H})$, $7.12(\mathrm{t}, J=8.3 \mathrm{~Hz}, 4 \mathrm{H}), 4.07-3.95(\mathrm{~m}, 1 \mathrm{H}), 3.85-3.72(\mathrm{~m}, 1 \mathrm{H}), 3.56(\mathrm{dd}, J=12.5,6.0 \mathrm{~Hz}, 1 \mathrm{H}), 2.78-2.59(\mathrm{~m}$, $4 \mathrm{H}), 2.01-1.60(\mathrm{~m}, 5 \mathrm{H}), 1.24(\mathrm{td}, J=7.0,2.7 \mathrm{~Hz}, 3 \mathrm{H}) .{ }^{13} \mathrm{C}-\mathrm{NMR}\left(101 \mathrm{MHz}, \mathrm{CDCl}_{3}\right) \delta=142.8(\mathrm{~s}), 139.9(\mathrm{~s})$, $132.1(\mathrm{~d}, J=2.7 \mathrm{~Hz}), 131.6(\mathrm{~s}), 131.5(\mathrm{~s}), 131.4-130.0(\mathrm{~m}), 128.6(\mathrm{~s}), 128.5(\mathrm{~d}, J=1.5 \mathrm{~Hz}), 128.4(\mathrm{~s}), 127.2(\mathrm{~d}$, $J=4.4 \mathrm{~Hz}), 127.1(\mathrm{~s}), 126.1(\mathrm{~s}), 63.4(\mathrm{~d}, J=17.4 \mathrm{~Hz}), 60.5(\mathrm{~d}, J=4.3 \mathrm{~Hz}), 48.6(\mathrm{~d}, J=3.4 \mathrm{~Hz}), 36.4(\mathrm{~s}), 29.6$ $(\mathrm{d}, J=2.8 \mathrm{~Hz}), 26.3(\mathrm{dd}, J=101.4,21.2 \mathrm{~Hz}), 16.4(\mathrm{~d}, J=6.3 \mathrm{~Hz})$. HRMS $\left(\mathrm{ESI}^{+}\right)$Calcd. for $\mathrm{C}_{25} \mathrm{H}_{30} \mathrm{NO}_{2} \mathrm{P}$ [M]: 408.2092, Found: 408.2107.

\section{Diethyl 3-butylamino-3-phenyl propylphosphonate, 11ca}

The pure 11ca was obtained as a yellow oil. Yield $86 \%(49 \mathrm{mg}) . R_{f}=0.36$ (ethyl acetate). ${ }^{31} \mathrm{P}-\mathrm{NMR}$ $\left(162 \mathrm{MHz}, \mathrm{CDCl}_{3}\right) \delta=32.49$ (s). ${ }^{1} \mathrm{H}-\mathrm{NMR}\left(400 \mathrm{MHz}, \mathrm{CDCl}_{3}\right) \delta=7.35-7.28(\mathrm{~m}, 2 \mathrm{H}), 7.25(\mathrm{~d}, J=7.3 \mathrm{~Hz}$, $3 \mathrm{H}), 4.10-3.90(\mathrm{~m}, 4 \mathrm{H}), 3.59(\mathrm{t}, J=6.7 \mathrm{~Hz}, 1 \mathrm{H}), 2.50-2.31(\mathrm{~m}, 2 \mathrm{H}), 1.93(\mathrm{tdd}, J=23.7,21.5,16.4 \mathrm{~Hz}, 3 \mathrm{H})$, $1.79-1.50(\mathrm{~m}, 3 \mathrm{H}), 1.40(\mathrm{dt}, J=11.9,7.0 \mathrm{~Hz}, 2 \mathrm{H}), 1.32-1.16(\mathrm{~m}, 8 \mathrm{H}), 0.86(\mathrm{t}, J=7.3 \mathrm{~Hz}, 3 \mathrm{H}) .{ }^{13} \mathrm{C}-\mathrm{NMR}$ $\left(101 \mathrm{MHz}, \mathrm{CDCl}_{3}\right) \delta=143.1(\mathrm{~s}), 128.5(\mathrm{~s}), 127.2(\mathrm{~s}), 127.1(\mathrm{~s}), 63.5(\mathrm{~d}, J=17.9 \mathrm{~Hz}), 61.5(\mathrm{~d}, J=1.4 \mathrm{~Hz})$, $61.4(\mathrm{~d}, J=1.2 \mathrm{~Hz}), 47.3(\mathrm{~s}), 32.3(\mathrm{~s}), 30.4(\mathrm{~d}, J=4.4 \mathrm{~Hz}), 23.0(\mathrm{~s}), 21.6(\mathrm{~s}), 20.4(\mathrm{~s}), 16.4(\mathrm{~d}, J=1.3 \mathrm{~Hz}), 16.3$ $(\mathrm{d}, J=2.0 \mathrm{~Hz}), 13.9$ (s). HRMS (ESI ${ }^{+}$) Calcd. for $\mathrm{C}_{17} \mathrm{H}_{30} \mathrm{NO}_{3} \mathrm{P}$ [M]: 328.2042, Found: 328.2083. 
Diethyl 3-phenylethylamino-3-phenyl propylphosphonate, $\mathbf{1 1} \mathrm{cb}$

The pure 11cb was obtained as a yellow oil. Yield $79 \%$ (51 mg). $R_{f}=0.85$ (petroleum ether:ethyl acetate $=1: 3) .{ }^{31} \mathrm{P}-\mathrm{NMR}\left(162 \mathrm{MHz}, \mathrm{CDCl}_{3}\right) \delta=32.38(\mathrm{~s}) .{ }^{1} \mathrm{H}-\mathrm{NMR}\left(400 \mathrm{MHz}, \mathrm{CDCl}_{3}\right) \delta=7.33-7.28$ $(\mathrm{m}, 2 \mathrm{H}), 7.25(\mathrm{dd}, J=6.5,3.2 \mathrm{~Hz}, 3 \mathrm{H}), 7.22-7.17(\mathrm{~m}, 3 \mathrm{H}), 7.14(\mathrm{~d}, J=6.9 \mathrm{~Hz}, 2 \mathrm{H}), 4.11-3.94(\mathrm{~m}, 4 \mathrm{H})$, 3.67-3.56 (m, 1H), 2.78-2.67 (m, 4H), 1.70-1.47 (m, 4H), $1.27(\mathrm{td}, J=7.0,3.5 \mathrm{~Hz}, 6 \mathrm{H}) .{ }^{13} \mathrm{C}-\mathrm{NMR}(101$ $\left.\mathrm{MHz}, \mathrm{CDCl}_{3}\right) \delta=142.8(\mathrm{~s}), 139.9(\mathrm{~s}), 128.7(\mathrm{~s}), 128.5(\mathrm{~s}), 128.4(\mathrm{~s}), 127.3(\mathrm{~s}), 127.1(\mathrm{~s}), 126.1(\mathrm{~s}), 110.0(\mathrm{~s})$, $63.3(\mathrm{~d}, J=17.8 \mathrm{~Hz}), 61.4(\mathrm{~d}, J=6.4 \mathrm{~Hz}), 48.6(\mathrm{~s}), 36.4(\mathrm{~s}), 30.4(\mathrm{~d}, J=4.3 \mathrm{~Hz}), 23.0(\mathrm{~s}), 21.6(\mathrm{~s}), 16.4(\mathrm{dd}$, $J=6.0,1.6 \mathrm{~Hz}$ ). HRMS (ESI ${ }^{+}$) Calcd. for $\mathrm{C}_{21} \mathrm{H}_{30} \mathrm{NO}_{3} \mathrm{P}[\mathrm{M}]: 376.2042$, Found: 376.2041 .

Dimenthyl 3-butylamino-3-phenyl propylphosphonate, 11da

The pure 11da was obtained as a yellow oil. Yield $77 \%(49 \mathrm{mg}) . R_{f}=0.78$ (petroleum ether:ethyl acetate $=1: 3) .{ }^{31} \mathrm{P}-\mathrm{NMR}\left(162 \mathrm{MHz}, \mathrm{CDCl}_{3}\right) \delta=30.07(\mathrm{~s}) .{ }^{1} \mathrm{H}-\mathrm{NMR}\left(400 \mathrm{MHz}, \mathrm{CDCl}_{3}\right) \delta=7.32(\mathrm{~d}, J=7.0$ $\mathrm{Hz}, 2 \mathrm{H}), 7.30-7.24(\mathrm{~m}, 3 \mathrm{H}), 4.21-4.04(\mathrm{~m}, 2 \mathrm{H}), 3.62(\mathrm{t}, J=6.7 \mathrm{~Hz}, 1 \mathrm{H}), 2.46(\mathrm{~d}, J=7.6 \mathrm{~Hz}, 2 \mathrm{H}), 2.21(\mathrm{~d}$, $J=11.4 \mathrm{~Hz}, 2 \mathrm{H}), 2.16-2.07(\mathrm{~m}, 1 \mathrm{H}), 1.97(\mathrm{ddd}, J=26.2,16.9,8.2 \mathrm{~Hz}, 3 \mathrm{H}), 1.77-1.51(\mathrm{~m}, 10 \mathrm{H}), 1.50-1.37$ $(\mathrm{m}, 5 \mathrm{H}), 1.37-1.22(\mathrm{~m}, 5 \mathrm{H}), 1.15-0.96(\mathrm{~m}, 2 \mathrm{H}), 0.91(\mathrm{dd}, J=5.2,2.0 \mathrm{~Hz}, 11 \mathrm{H}), 0.88(\mathrm{dd}, J=6.9,3.7 \mathrm{~Hz}$, $3 \mathrm{H}), 0.81-0.78(\mathrm{~m}, 3 \mathrm{H}), 0.74(\mathrm{~d}, J=6.9,1 \mathrm{H}), 0.69(\mathrm{~d}, J=6.9,1 \mathrm{H}) .{ }^{13} \mathrm{C}-\mathrm{NMR}\left(101 \mathrm{MHz}, \mathrm{CDCl}_{3}\right) \delta=143.3$ $(\mathrm{s}), 128.4(\mathrm{~s}), 127.2(\mathrm{dd}, J=8.8,5.8 \mathrm{~Hz}), 77.3(\mathrm{~s}), 77.1-76.5(\mathrm{~m}), 63.7(\mathrm{~d}, J=18.0 \mathrm{~Hz}), 48.6(\mathrm{~d}, J=6.5 \mathrm{~Hz})$, $47.4(\mathrm{~s}), 43.7(\mathrm{~d}, J=7.9 \mathrm{~Hz}), 43.0(\mathrm{~d}, J=15.4 \mathrm{~Hz}), 34.1(\mathrm{~s}), 32.4(\mathrm{~s}), 31.6-31.2(\mathrm{~m}), 30.9(\mathrm{~s}), 25.6-25.1(\mathrm{~m})$, $23.7(\mathrm{~s}), 22.7(\mathrm{t}, J=5.6 \mathrm{~Hz}), 21.9(\mathrm{~s}), 21.03(\mathrm{~s}), 20.0(\mathrm{~s}), 15.7(\mathrm{dd}, J=26.2,7.1 \mathrm{~Hz}), 13.9(\mathrm{~s})$. HRMS $\left(\mathrm{ESI}^{+}\right)$ Calcd. for $\mathrm{C}_{33} \mathrm{H}_{58} \mathrm{NO}_{3} \mathrm{P}\left[\mathrm{M}+\mathrm{H}^{+}\right]$: 548.4233, Found: 548.4224 .

\section{Dimenthyl 3-phenylethylamino-3-phenyl propylphosphonate, 11db}

The pure $11 \mathrm{db}$ was obtained as a yellow oil. Yield $77 \%(49 \mathrm{mg}) \cdot R_{f}=0.66$ (petroleum ether:ethyl acetate $=1: 3) .{ }^{31} \mathrm{P}-\mathrm{NMR}\left(162 \mathrm{MHz}, \mathrm{CDCl}_{3}\right) \delta=29.96(\mathrm{~s}, 50 \%), 29.94(\mathrm{~s}, 50 \%) .{ }^{1} \mathrm{H}-\mathrm{NMR}(400 \mathrm{MHz}$, $\left.\mathrm{CDCl}_{3}\right) \delta=7.29(\mathrm{~d}, J=7.3 \mathrm{~Hz}, 3 \mathrm{H}), 7.23(\mathrm{~d}, J=5.6 \mathrm{~Hz}, 2 \mathrm{H}), 7.20(\mathrm{~d}, J=8.0 \mathrm{~Hz}, 3 \mathrm{H}), 7.14(\mathrm{~d}, J=8.0 \mathrm{~Hz}$, 2H), 4.17-4.03 (m, 2H), $3.61(\mathrm{dd}, J=13.1,5.4 \mathrm{~Hz}, 1 \mathrm{H}), 2.73(\mathrm{t}, J=6.3 \mathrm{~Hz}, 4 \mathrm{H}), 2.12(\mathrm{~d}, J=34.5 \mathrm{~Hz}, 3 \mathrm{H})$, $1.94(\mathrm{dd}, J=13.8,7.0 \mathrm{~Hz}, 2 \mathrm{H}), 1.69-1.35(\mathrm{~m}, 10 \mathrm{H}), 1.25(\mathrm{~s}, 2 \mathrm{H}), 1.14-1.02(\mathrm{~m}, 2 \mathrm{H}), 0.95(\mathrm{dd}, J=21.1,9.0$ $\mathrm{Hz}, 3 \mathrm{H}), 0.91-0.84(\mathrm{~m}, 12 \mathrm{H}), 0.78(\mathrm{t}, J=8.4 \mathrm{~Hz}, 4 \mathrm{H}), 0.70(\mathrm{~d}, J=6.9 \mathrm{~Hz}, 1 \mathrm{H}), 0.65(\mathrm{~d}, J=6.9 \mathrm{~Hz}, 1 \mathrm{H})$. ${ }^{13} \mathrm{C}-\mathrm{NMR}\left(101 \mathrm{MHz}, \mathrm{CDCl}_{3}\right) \delta=142.9(\mathrm{~s}), 140.0(\mathrm{~s}), 128.7-128.3(\mathrm{~m}), 127.2(\mathrm{~d}, J=6.7 \mathrm{~Hz}), 126.1(\mathrm{~s}), 77.3$ $(\mathrm{s}), 77.0(\mathrm{~s}), 76.7(\mathrm{~s}), 63.5(\mathrm{~d}, J=17.8 \mathrm{~Hz}), 49.1-48.1(\mathrm{~m}), 43.7(\mathrm{~d}, J=8.2 \mathrm{~Hz}), 43.0(\mathrm{~d}, J=15.8 \mathrm{~Hz}), 36.4(\mathrm{~s})$, $34.1(\mathrm{~s}), 32.0-31.3(\mathrm{~m}), 30.9(\mathrm{~s}), 29.7(\mathrm{~s}), 25.3(\mathrm{t}, J=18.1 \mathrm{~Hz}), 23.6(\mathrm{~d}, J=11.0 \mathrm{~Hz}), 22.7(\mathrm{t}, J=5.5 \mathrm{~Hz}), 22.0$ $(\mathrm{d}, J=3.5 \mathrm{~Hz}), 21.1(\mathrm{~s}), 15.8(\mathrm{~d}, J=5.7 \mathrm{~Hz}), 15.5(\mathrm{~d}, J=7.7 \mathrm{~Hz})$. HRMS $\left(\mathrm{ESI}^{+}\right)$Calcd. for $\mathrm{C}_{37} \mathrm{H}_{58} \mathrm{NO}_{3} \mathrm{P}$ $\left[\mathrm{M}+\mathrm{H}^{+}\right]$: 596.4233, Found: 596.4224 .

Dimenthyl 3-phenyl-3-pyrrolidin-1-yl propylphosphonate, 11dc

The pure 11dc was obtained as a yellow oil. Yield $77 \%(49 \mathrm{mg}) . R_{f}=0.80$ (petroleum ether:ethyl acetate $=1: 3) .{ }^{31} \mathrm{P}-\mathrm{NMR}\left(162 \mathrm{MHz}, \mathrm{CDCl}_{3}\right) \delta=30.23(\mathrm{~s}, 61 \%), 30.19(\mathrm{~s}, 39 \%) .{ }^{1} \mathrm{H}-\mathrm{NMR}(400 \mathrm{MHz}$, $\left.\mathrm{CDCl}_{3}\right) \delta=7.26(\mathrm{t}, J=13.1 \mathrm{~Hz}, 5 \mathrm{H}), 4.15-4.02(\mathrm{~m}, 2 \mathrm{H}), 3.08(\mathrm{~d}, J=5.7 \mathrm{~Hz}, 1 \mathrm{H}), 2.53(\mathrm{~s}, 1 \mathrm{H}), 2.36(\mathrm{~s}$, 2H), $2.15(\mathrm{~s}, 3 \mathrm{H}), 1.99(\mathrm{~s}, 2 \mathrm{H}), 1.67(\mathrm{dd}, J=32.6,15.7 \mathrm{~Hz}, 11 \mathrm{H}), 1.41(\mathrm{~s}, 4 \mathrm{H}), 1.25(\mathrm{~s}, 4 \mathrm{H}), 1.07(\mathrm{dd}$, $J=22.6,10.9 \mathrm{~Hz}, 2 \mathrm{H}), 0.87(\mathrm{dd}, J=11.9,6.6 \mathrm{~Hz}, 12 \mathrm{H}), 0.79-0.75(\mathrm{~m}, 3 \mathrm{H}), 0.71(\mathrm{~d}, J=7.0 \mathrm{~Hz}, 2 \mathrm{H}), 0.63(\mathrm{~d}$, $J=6.9 \mathrm{~Hz}, 2 \mathrm{H}) .{ }^{13} \mathrm{C}-\mathrm{NMR}\left(101 \mathrm{MHz}, \mathrm{CDCl}_{3}\right) \delta=142.1(\mathrm{~s}), 128.3-127.9(\mathrm{~m}), 127.1(\mathrm{~s}), 77.3(\mathrm{~s}), 77.0(\mathrm{~s})$, $76.7(\mathrm{~s}), 71.1(\mathrm{~s}), 52.7(\mathrm{~s}), 48.5(\mathrm{~s}), 43.8(\mathrm{~s}), 43.0(\mathrm{~d}, J=18.4 \mathrm{~Hz}), 34.1(\mathrm{~s}), 31.5(\mathrm{~d}, J=4.6 \mathrm{~Hz}), 29.7(\mathrm{~s}), 29.0$ (s), 25.7-24.8 (m), $23.3(\mathrm{~s}), 22.7(\mathrm{~s}), 21.9(\mathrm{~s}), 21.1(\mathrm{~s}), 15.6(\mathrm{dd}, J=25.7,12.3 \mathrm{~Hz})$. HRMS (ESI $\left.{ }^{+}\right)$Calcd. for $\mathrm{C}_{33} \mathrm{H}_{56} \mathrm{NO}_{3} \mathrm{P}\left[\mathrm{M}+\mathrm{H}^{+}\right.$]: 546.4076, Found: 546.4069.

\section{Dimenthyl-3-methyl phenylamino-3-phenyl propylphosphonate, 11dd}

The pure 11dd was obtained as a yellow oil. Yield $62 \%(35 \mathrm{mg}) . R_{f}=0.74$ (petroleum ether:ethyl acetate $=1: 3) .{ }^{31} \mathrm{P}-\mathrm{NMR}\left(162 \mathrm{MHz}, \mathrm{CDCl}_{3}\right) \delta=30.28(\mathrm{~s}, 50 \%), 30.26(\mathrm{~s}, 50 \%) .{ }^{1} \mathrm{H}-\mathrm{NMR}(400 \mathrm{MHz}$, $\left.\mathrm{CDCl}_{3}\right) \delta=7.36-7.28(\mathrm{~m}, 3 \mathrm{H}), 7.23(\mathrm{dd}, J=18.7,7.4 \mathrm{~Hz}, 6 \mathrm{H}), 7.13(\mathrm{~d}, J=7.4 \mathrm{~Hz}, 1 \mathrm{H}), 4.10(\mathrm{~s}, 2 \mathrm{H}), 3.68$ $(\mathrm{dd}, J=29.0,6.3 \mathrm{~Hz}, 1 \mathrm{H}), 3.48-3.24(\mathrm{~m}, 1 \mathrm{H}), 2.13(\mathrm{~d}, J=41.4 \mathrm{~Hz}, 2 \mathrm{H}), 2.00(\mathrm{~s}, 1 \mathrm{H}), 1.89-1.79(\mathrm{~m}, 1 \mathrm{H})$, 
$1.63(\mathrm{~d}, J=10.8 \mathrm{~Hz}, 8 \mathrm{H}), 1.40(\mathrm{~s}, 2 \mathrm{H}), 1.32(\mathrm{~d}, J=6.3 \mathrm{~Hz}, 1 \mathrm{H}), 1.24(\mathrm{~d}, J=7.0 \mathrm{~Hz}, 4 \mathrm{H}), 1.10-0.97(\mathrm{~m}$, $2 \mathrm{H}), 0.87(\mathrm{~d}, J=6.3 \mathrm{~Hz}, 12 \mathrm{H}), 0.82(\mathrm{~d}, J=11.6 \mathrm{~Hz}, 2 \mathrm{H}), 0.77(\mathrm{t}, J=7.1 \mathrm{~Hz}, 3 \mathrm{H}), 0.70(\mathrm{~d}, J=6.5 \mathrm{~Hz}, 3 \mathrm{H})$. ${ }^{13} \mathrm{C}-\mathrm{NMR}\left(101 \mathrm{MHz}, \mathrm{CDCl}_{3}\right) \delta=128.4(\mathrm{~d}, J=9.3 \mathrm{~Hz}), 127.4-126.6(\mathrm{~m}), 126.5(\mathrm{~s}), 77.3(\mathrm{~s}), 77.1-76.5(\mathrm{~m})$, $60.2(\mathrm{~d}, J=18.3 \mathrm{~Hz}), 54.6(\mathrm{~s}), 48.5(\mathrm{~s}), 43.7(\mathrm{~s}), 43.0(\mathrm{~d}, J=9.3 \mathrm{~Hz}), 34.1(\mathrm{~s}), 31.5(\mathrm{~d}, J=10.2 \mathrm{~Hz}), 30.2(\mathrm{~s})$, $29.7(\mathrm{~s}), 25.4(\mathrm{dd}, J=28.7,10.2 \mathrm{~Hz}), 23.0-22.6(\mathrm{~m}), 22.3(\mathrm{~d}, J=12.6 \mathrm{~Hz}), 22.0(\mathrm{~d}, J=2.9 \mathrm{~Hz}), 21.1(\mathrm{~s})$, $15.8(\mathrm{~d}, J=7.0 \mathrm{~Hz}), 15.6(\mathrm{~s}), 14.0(\mathrm{~s})$. HRMS $\left(\mathrm{ESI}^{+}\right)$Calcd. for $\mathrm{C}_{36} \mathrm{H}_{56} \mathrm{NO}_{3} \mathrm{P}\left[\mathrm{M}+\mathrm{H}^{+}\right]: 582.4076$, Found: 582.4080 .

\section{Diethyl 3-butylamino-3-p-tolyl propylphosphonate, 11ea}

The pure 11ea was obtained as a yellow oil. Yield $83 \%(47 \mathrm{mg}) . \quad R_{f}=0.70$ (dichloromethane/methanol = 20:1). ${ }^{31} \mathrm{P}-\mathrm{NMR}\left(162 \mathrm{MHz}, \mathrm{CDCl}_{3}\right) \delta=32.57$ (s). ${ }^{1} \mathrm{H}-\mathrm{NMR}(400 \mathrm{MHz}$, $\left.\mathrm{CDCl}_{3}\right) \delta=7.12(\mathrm{~d}, J=8.6 \mathrm{~Hz}, 4 \mathrm{H}), 4.09-3.97(\mathrm{~m}, 4 \mathrm{H}), 3.56(\mathrm{t}, J=6.7 \mathrm{~Hz}, 1 \mathrm{H}), 2.51-2.38(\mathrm{~m}, 2 \mathrm{H}), 2.33(\mathrm{~s}$, $3 \mathrm{H}), 2.04-1.79(\mathrm{~m}, 2 \mathrm{H}), 1.64(\mathrm{dddd}, J=26.4,19.3,15.4,4.8 \mathrm{~Hz}, 3 \mathrm{H}), 1.48-1.36(\mathrm{~m}, 2 \mathrm{H}), 1.34-1.21(\mathrm{~m}, 8 \mathrm{H})$, $0.86(\mathrm{t}, J=7.2 \mathrm{~Hz}, 3 \mathrm{H}) .{ }^{13} \mathrm{C}-\mathrm{NMR}\left(101 \mathrm{MHz}, \mathrm{CDCl}_{3}\right) \delta=140.1(\mathrm{~s}), 136.7(\mathrm{~s}), 129.1(\mathrm{~s}), 127.0(\mathrm{~s}), 63.2(\mathrm{~d}$, $J=17.9 \mathrm{~Hz}), 61.4(\mathrm{~d}, J=2.2 \mathrm{~Hz}), 61.3(\mathrm{~d}, J=2.1 \mathrm{~Hz}), 53.4(\mathrm{~s}), 47.3(\mathrm{~s}), 32.4(\mathrm{~s}), 30.4(\mathrm{~d}, J=4.3 \mathrm{~Hz}), 29.6(\mathrm{~s})$, $23.1(\mathrm{~s}), 21.7(\mathrm{~s}), 21.0(\mathrm{~s}), 20.4(\mathrm{~s}), 16.4(\mathrm{~d}, J=6.0 \mathrm{~Hz}), 13.9(\mathrm{~s})$. HRMS (ESI $\left.{ }^{+}\right)$Calcd. for $\mathrm{C}_{18} \mathrm{H}_{32} \mathrm{NO}_{3} \mathrm{P}[\mathrm{M}]$ : 342.2198, Found: 342.2234.

\section{Diethyl 3-phenethylamino-3-p-tolyl propylphosphonate, 11eb}

The pure 11eb was obtained as a yellow oil. Yield $77 \%(49 \mathrm{mg}) . \quad R_{f}=0.65$ (dichloromethane/methanol = 20:1). ${ }^{31} \mathrm{P}-\mathrm{NMR}\left(162 \mathrm{MHz}, \mathrm{CDCl}_{3}\right) \delta=32.46$ (s). ${ }^{1} \mathrm{H}-\mathrm{NMR}(400 \mathrm{MHz}$, $\left.\mathrm{CDCl}_{3}\right) \delta=7.25(\mathrm{dd}, J=8.4,5.8 \mathrm{~Hz}, 2 \mathrm{H}), 7.22-7.05(\mathrm{~m}, 7 \mathrm{H}), 4.26-3.89(\mathrm{~m}, 4 \mathrm{H}), 3.57(\mathrm{t}, J=6.7 \mathrm{~Hz}$, $1 \mathrm{H}), 2.95-2.55(\mathrm{~m}, 4 \mathrm{H}), 2.32(\mathrm{~s}, 3 \mathrm{H}), 2.29-1.81(\mathrm{~m}, 2 \mathrm{H}), 1.74-1.47(\mathrm{~m}, 3 \mathrm{H}), 1.26(\mathrm{td}, J=7.0,3.1 \mathrm{~Hz}, 6 \mathrm{H})$. ${ }^{13} \mathrm{C}-\mathrm{NMR}\left(101 \mathrm{MHz}, \mathrm{CDCl}_{3}\right) \delta=139.9(\mathrm{~d}, J=21.9 \mathrm{~Hz}), 136.8(\mathrm{~s}), 129.1(\mathrm{~s}), 128.6(\mathrm{~s}), 128.3(\mathrm{~s}), 127.0(\mathrm{~s})$, $126.0(\mathrm{~s}), 63.0(\mathrm{~d}, J=17.9 \mathrm{~Hz}), 61.4(\mathrm{~d}, J=1.1 \mathrm{~Hz}), 61.3(\mathrm{~d}, J=1.1 \mathrm{~Hz}), 53.4(\mathrm{~s}), 48.6(\mathrm{~s}), 36.4(\mathrm{~s}), 30.4(\mathrm{~d}$, $J=4.3 \mathrm{~Hz}), 23.0(\mathrm{~s}), 21.6(\mathrm{~s}), 21.0(\mathrm{~s}), 16.4(\mathrm{~d}, J=0.7 \mathrm{~Hz})$. HRMS $\left(\mathrm{ESI}^{+}\right)$Calcd. for $\mathrm{C}_{22} \mathrm{H}_{32} \mathrm{NO}_{3} \mathrm{P}[\mathrm{M}]$ : 391.2276, Found: 391.2232.

\section{Diphenyl 3-butylamino-3-phenylpropyl phosphine oxide, 11fa}

The pure 11fa was obtained as a yellow oil. Yield $80 \%(44 \mathrm{mg}) . \quad R_{f}=0.50$ (dichloromethane/methanol = 20:1). ${ }^{31} \mathrm{P}-\mathrm{NMR}\left(162 \mathrm{MHz}, \mathrm{CDCl}_{3}\right) \delta=33.05$ (s). ${ }^{1} \mathrm{H}-\mathrm{NMR}(400 \mathrm{MHz}$, $\left.\mathrm{CDCl}_{3}\right) \delta=7.62(\mathrm{dd}, J=10.8,7.9 \mathrm{~Hz}, 4 \mathrm{H}), 7.52-7.45(\mathrm{~m}, 2 \mathrm{H}), 7.41(\mathrm{qd}, J=7.1,3.5 \mathrm{~Hz}, 4 \mathrm{H}), 7.31(\mathrm{t}$, $J=7.2 \mathrm{~Hz}, 2 \mathrm{H}), 7.28-7.24(\mathrm{~m}, 1 \mathrm{H}), 7.21(\mathrm{t}, J=6.4 \mathrm{~Hz}, 2 \mathrm{H}), 3.66-3.58(\mathrm{~m}, 1 \mathrm{H}), 2.45-2.32(\mathrm{~m}, 2 \mathrm{H}), 2.30-2.18$ $(\mathrm{m}, 1 \mathrm{H}), 2.18-1.96(\mathrm{~m}, 3 \mathrm{H}), 1.91(\mathrm{ddd}, J=20.6,12.6,6.4 \mathrm{~Hz}, 1 \mathrm{H}), 1.44-1.32(\mathrm{~m}, 2 \mathrm{H}), 1.31-1.18(\mathrm{~m}, 2 \mathrm{H})$, $0.84(\mathrm{t}, J=7.3 \mathrm{~Hz}, 3 \mathrm{H}) .{ }^{13} \mathrm{C}-\mathrm{NMR}\left(101 \mathrm{MHz}, \mathrm{CDCl}_{3}\right) \delta=143.0(\mathrm{~s}), 132.9(\mathrm{dd}, J=98.4,46.3 \mathrm{~Hz}), 131.6(\mathrm{~s})$, $130.8(\mathrm{~s}), 130.7(\mathrm{~d}, J=2.2 \mathrm{~Hz}), 130.6(\mathrm{~s}), 128.6(\mathrm{~s}), 128.5(\mathrm{~d}, J=4.3 \mathrm{~Hz}), 127.2(\mathrm{~s}), 63.6(\mathrm{~d}, J=14.6 \mathrm{~Hz})$, 47.3 (s), $32.3(\mathrm{~s}), 29.3(\mathrm{~d}, J=3.0 \mathrm{~Hz}), 26.0(\mathrm{~d}, J=72.6 \mathrm{~Hz}), 20.4(\mathrm{~s}), 14.0(\mathrm{~s})$. HRMS (ESI ${ }^{+}$) Calcd. for $\mathrm{C}_{25} \mathrm{H}_{30} \mathrm{NOP}$ [M]: 392.2143, Found: 392.2147 .

To a solution of $1 \mathrm{a}(1.02 \mathrm{~g}, 2.89 \mathrm{mmol})$, palladium acetate $(32.5 \mathrm{mg}, 0.145 \mathrm{mmol}, 5 \mathrm{~mol} \%)$, and triphenyl phosphine $(0.152 \mathrm{~g}, 0.580 \mathrm{mmol}, 20 \mathrm{~mol} \%)$ in toluene $(2 \mathrm{~mL}), 3 \mathrm{a}(0.63 \mathrm{~mL}, 6.36 \mathrm{mmol})$ was added. The mixture was heated at $120^{\circ} \mathrm{C}$ for $24 \mathrm{~h}$ and monitored with TLC. After the reaction was completed, the mixture was cooled to room temperature. Ethanol $(5 \mathrm{~mL})$ and sodium borohydride $(0.44 \mathrm{~g}, 11.6 \mathrm{mmol})$ were added. The mixture was stirred at room temperature for $14 \mathrm{~h}$. After a saturated solution of ammonium chloride $(10 \mathrm{~mL})$ was added, the mixture was extracted with dichloromethane $(3 \times 15 \mathrm{~mL})$ and washed with water $(3 \times 10 \mathrm{~mL})$. The crude product was obtained as a red oil in a $66 \%$ yield (estimated by a ${ }^{31} \mathrm{P}-\mathrm{NMR}$ spectrum). The residue was dissolved in ethanol ( $5 \mathrm{~mL}$ ), acidified to $\mathrm{pH}=2$ with diluted hydrochloric acid $(\mathrm{ca} .7 \%, 6 \mathrm{~mL})$, and then the ethanol was removed in vacuo. The residue was washed with ether $(5 \times 5 \mathrm{~mL})$, and the aqueous phase was neutralized with saturated sodium bicarbonate solution $(10 \mathrm{~mL})$ until $\mathrm{pH}=9$. The mixture was extracted with dichloromethane $(3 \times 15 \mathrm{~mL})$. After drying and removal of the solvent, 11fa was obtained as a yellow oil. Yield 59\% $(0.67 \mathrm{~g}) .11 \mathrm{fa}$ gave similar spectrum data to those above. 
Diphenyl 3-phenethylamino-3-phenyl propylphosphine oxide, 11fb

The pure 11fb was obtained as a yellow oil. Yield $82 \%(51 \mathrm{mg}) . \quad R_{f}=0.53$ (dichloromethane $/$ methanol $=15: 1) .{ }^{31} \mathrm{P}-\mathrm{NMR} \delta=33.02(\mathrm{~s}) .{ }^{1} \mathrm{H}-\mathrm{NMR}\left(400 \mathrm{MHz}, \mathrm{CDCl}_{3}\right) \delta=7.60(\mathrm{dd}$, $J=10.0,8.5 \mathrm{~Hz}, 4 \mathrm{H}), 7.54-7.45(\mathrm{~m}, 2 \mathrm{H}), 7.41(\mathrm{t}, J=7.2 \mathrm{~Hz}, 4 \mathrm{H}), 7.33-7.21(\mathrm{~m}, 5 \mathrm{H}), 7.20-7.08(\mathrm{~m}, 5 \mathrm{H}), 3.63$ $(\mathrm{t}, J=6.4 \mathrm{~Hz}, 1 \mathrm{H}), 2.81-2.60(\mathrm{~m}, 4 \mathrm{H}), 2.28-2.15(\mathrm{~m}, 1 \mathrm{H}), 2.14-2.04(\mathrm{~m}, 1 \mathrm{H}), 2.04-1.94(\mathrm{~m}, 1 \mathrm{H}), 1.94-1.81$ $(\mathrm{m}, 1 \mathrm{H}) .{ }^{13} \mathrm{C}-\mathrm{NMR}\left(101 \mathrm{MHz}, \mathrm{CDCl}_{3}\right) \delta=142.7(\mathrm{~s}), 139.9(\mathrm{~s}), 133.3(\mathrm{~d}, J=43.1 \mathrm{~Hz}), 132.4(\mathrm{~d}, J=43.0$ Hz), 131.6 (s), 130.8 (s), 130.7 (s), 130.6 (s), 128.7 (d, J = 2.7 Hz), 128.5 (d, J = 2.1 Hz), 128.4 (s), 127.3 $(\mathrm{d}, J=9.0 \mathrm{~Hz}), 126.1(\mathrm{~s}), 63.4(\mathrm{~d}, J=14.6 \mathrm{~Hz}), 48.5(\mathrm{~s}), 36.3(\mathrm{~s}), 29.3(\mathrm{~d}, J=3.3 \mathrm{~Hz}), 26.0(\mathrm{~d}, J=72.6 \mathrm{~Hz})$. HRMS (ESI ${ }^{+}$) Calcd. for $\mathrm{C}_{29} \mathrm{H}_{30}$ NOP [M]: 440.2143, Found: 440.2142 .

$\left(S_{P}\right)$-Menthyl-3-butylamino-3-phenylpropyl phenylphosphine oxide, $\mathbf{1 1 g a}_{\mathbf{A}} / \mathbf{1 1} \mathbf{g a}_{\mathbf{B}}$

Compound 11ga $\mathbf{A} / 11 g a_{B}$ was obtained as a yellow oil. Yield 85\% (56 mg). $\quad R_{f}=0.46$ (dichloromethane/methanol = 15:1). ${ }^{31} \mathrm{P}-\mathrm{NMR}\left(162 \mathrm{MHz}, \mathrm{CDCl}_{3}\right) \delta=43.39$ (s). ${ }^{1} \mathrm{H}-\mathrm{NMR}(400 \mathrm{MHz}$, $\left.\mathrm{CDCl}_{3}\right) \delta=7.58-7.51(\mathrm{~m}, 2 \mathrm{H}), 7.48-7.39(\mathrm{~m}, 3 \mathrm{H}), 7.29(\mathrm{dd}, J=13.9,6.5 \mathrm{~Hz}, 3 \mathrm{H}), 7.16(\mathrm{~d}, J=6.7 \mathrm{~Hz}$, $2 \mathrm{H}), 3.59-3.51(\mathrm{~m}, 1 \mathrm{H}), 2.43-2.27(\mathrm{~m}, 2 \mathrm{H}), 2.13-1.89(\mathrm{~m}, 5 \mathrm{H}), 1.88-1.77(\mathrm{~m}, 1 \mathrm{H}), 1.67(\mathrm{~d}, J=10.8 \mathrm{~Hz}$, $3 \mathrm{H}), 1.54-1.42(\mathrm{~m}, 2 \mathrm{H}), 1.39-1.19(\mathrm{~m}, 5 \mathrm{H}), 1.17-1.03(\mathrm{~m}, 2 \mathrm{H}), 0.95(\mathrm{dd}, J=23.2,12.7 \mathrm{~Hz}, 2 \mathrm{H}), 0.86(\mathrm{~d}$, $J=6.6 \mathrm{~Hz}, 4 \mathrm{H}), 0.82(\mathrm{~d}, J=7.2 \mathrm{~Hz}, 2 \mathrm{H}), 0.78(\mathrm{~d}, J=6.8 \mathrm{~Hz}, 2 \mathrm{H}), 0.29(\mathrm{~d}, J=6.7 \mathrm{~Hz}, 3 \mathrm{H}) .{ }^{13} \mathrm{C}-\mathrm{NMR}(101$ $\left.\mathrm{MHz}, \mathrm{CDCl}_{3}\right) \delta=143.2(\mathrm{~s}), 134.2(\mathrm{~d}, J=87.4 \mathrm{~Hz}), 130.8(\mathrm{~d}, J=2.6 \mathrm{~Hz}), 130.5(\mathrm{~d}, J=8.4 \mathrm{~Hz}), 128.4-128.2$ $(\mathrm{m}), 127.4(\mathrm{~s}), 127.1(\mathrm{~s}), 63.9(\mathrm{~d}, J=13.6 \mathrm{~Hz}), 53.4(\mathrm{~s}), 47.3(\mathrm{~s}), 43.1(\mathrm{~s}), 41.1(\mathrm{~s}), 40.4(\mathrm{~s}), 35.1(\mathrm{~s}), 34.2(\mathrm{~s})$, $33.1(\mathrm{~d}, J=14.7 \mathrm{~Hz}), 32.2(\mathrm{~s}), 29.1(\mathrm{~s}), 28.1(\mathrm{~s}), 24.6(\mathrm{~s}), 24.3(\mathrm{~d}, J=2.3 \mathrm{~Hz}), 23.9(\mathrm{~s}), 22.5(\mathrm{~s}), 21.5(\mathrm{~s}), 20.3$ (s), 15.1 (s), 13.9 (s). HRMS (ESI ${ }^{+}$) Calcd. for $\mathrm{C}_{29} \mathrm{H}_{44} \mathrm{NOP}$ [M]: 454.3239, Found: 454.3248.

\section{$\left(S_{P}\right)$-Menthyl 3-phenethylamino-3-phenylpropyl phenylphosphine oxide, $\mathbf{1 1} \mathbf{g} \mathbf{b}_{\mathbf{A}} / \mathbf{1 1 g b}_{\mathbf{B}}, \mathbf{1 1 g b}_{\mathbf{A}}{ }^{\prime} / \mathbf{1 1 g b}_{\mathbf{B}}{ }^{\prime}$}

Compound $\mathbf{1 1 g b}_{\mathbf{A}} / \mathbf{1 1 g b}_{\mathrm{B}}, \mathbf{1 1 g b}_{\mathrm{A}}{ }^{\prime} / \mathbf{1 1 g b}_{\mathrm{B}}{ }^{\prime}$ was obtained as a yellow oil. Yield $87 \%$ (52 $\left.\mathrm{mg}\right) . R_{f}$ $=0.41$ (dichloromethane/methanol $=20: 1) .{ }^{31} \mathrm{P}-\mathrm{NMR}\left(162 \mathrm{MHz}, \mathrm{CDCl}_{3}\right) \delta=45.80(\mathrm{~s}, 17 \%), 45.34(\mathrm{~s}$, $23 \%), 43.44$ (s, 31\%), 43.28 (s, 29\%). ${ }^{1} \mathrm{H}-\mathrm{NMR}\left(400 \mathrm{MHz}, \mathrm{CDCl}_{3}\right) \delta=7.62-7.48(\mathrm{~m}, 3 \mathrm{H}), 7.43(\mathrm{dd}, J=7.4$, $5.8 \mathrm{~Hz}, 2 \mathrm{H}), 7.35-7.22(\mathrm{~m}, 5 \mathrm{H}), 7.21-7.07(\mathrm{~m}, 5 \mathrm{H}), 3.65-3.53(\mathrm{~m}, 1 \mathrm{H}), 2.79-2.46(\mathrm{~m}, 4 \mathrm{H}), 2.10-1.81(\mathrm{~m}$, $4 \mathrm{H}), 1.78-1.41(\mathrm{~m}, 7 \mathrm{H}), 1.26(\mathrm{~s}, 2 \mathrm{H}), 1.16-0.94(\mathrm{~m}, 2 \mathrm{H}), 0.87(\mathrm{dd}, J=8.6,6.5 \mathrm{~Hz}, 2 \mathrm{H}), 0.78(\mathrm{dd}, J=9.3,4.7$ $\mathrm{Hz}, 4 \mathrm{H}), 0.75-0.70(\mathrm{~m}, 1 \mathrm{H}), 0.31(\mathrm{dd}, J=10.9,6.8 \mathrm{~Hz}, 2 \mathrm{H}) .{ }^{13} \mathrm{C}-\mathrm{NMR}\left(101 \mathrm{MHz}, \mathrm{CDCl}_{3}\right) \delta=143.0(\mathrm{dd}$, $J=8.5,6.1 \mathrm{~Hz}), 140.2-139.9(\mathrm{~m}), 134.8-134.4(\mathrm{~m}), 133.8(\mathrm{~d}, J=20.0 \mathrm{~Hz}), 131.2-130.9(\mathrm{~m}), 130.8(\mathrm{~d}, J=3.3$ $\mathrm{Hz}), 130.5(\mathrm{dd}, J=8.5,2.4 \mathrm{~Hz}), 128.6(\mathrm{~d}, J=4.4 \mathrm{~Hz}), 128.4(\mathrm{~d}, J=1.3 \mathrm{~Hz}), 128.3(\mathrm{~s}), 128.2(\mathrm{~s}), 128.0(\mathrm{~d}$, $J=4.2 \mathrm{~Hz}), 127.4(\mathrm{~s}), 127.2-127.1(\mathrm{~m}), 127.0(\mathrm{~d}, J=3.8 \mathrm{~Hz}), 126.0(\mathrm{~d}, J=1.5 \mathrm{~Hz}), 63.7-63.1(\mathrm{~m}), 48.5(\mathrm{dd}$, $J=13.3,7.8 \mathrm{~Hz}), 43.5-43.1(\mathrm{~m}), 41.6-40.4(\mathrm{~m}), 37.0-36.8(\mathrm{~m}), 36.2(\mathrm{t}, J=11.7 \mathrm{~Hz}), 35.2(\mathrm{dd}, J=15.5,2.3$ Hz), $34.2(\mathrm{~s}), 33.2(\mathrm{ddd}, J=13.3,7.4,4.1 \mathrm{~Hz}), 29.7(\mathrm{~s}), 29.6-29.0(\mathrm{~m}), 28.3(\mathrm{dd}, J=22.1,5.1 \mathrm{~Hz}), 25.3(\mathrm{~d}$, $J=4.4 \mathrm{~Hz}), 24.8-24.5(\mathrm{~m}), 24.1(\mathrm{~d}, J=66.2 \mathrm{~Hz}), 22.5(\mathrm{~d}, J=3.3 \mathrm{~Hz}), 21.5-21.2(\mathrm{~m}), 15.7-15.0(\mathrm{~m})$. HRMS $\left(\mathrm{ESI}^{+}\right)$Calcd. for $\mathrm{C}_{33} \mathrm{H}_{44} \mathrm{NOP}[\mathrm{M}]: 502.3239$, Found: 502.3238 .

\subsubsection{Preparation of $\gamma$-Hydroxy Phosphorous Derivatives $\mathbf{1 2}$}

\section{Diphenyl 3-hydroxy-3-phenylprop-1-en-1-ylphosphine oxide, 12a}

To a solution of $\mathbf{1 f}(50 \mathrm{mg}, 0.142 \mathrm{mmol})$, palladium acetate $(1.6 \mathrm{mg}, 0.007 \mathrm{mmol})$, and triphenyl phosphine $(7.4 \mathrm{mg}, 0.284 \mathrm{mmol})$ in toluene $(0.5 \mathrm{~mL}), 3 \mathbf{a}(31 \mu \mathrm{L}, 0.312 \mathrm{mmol})$ was added. The mixture was heated at $120^{\circ} \mathrm{C}$ for $24 \mathrm{~h}$ and monitored with TLC, and then cooled to room temperature. Water $(3 \mu \mathrm{L}, 0.142 \mathrm{mmol})$ and sodium borohydride $(10.8 \mathrm{mg}, 0.284 \mathrm{mmol})$ were added. The mixture was stirred at room temperature for $13 \mathrm{~h}$. After a saturated solution of ammonium chloride $(3 \mathrm{~mL})$ was added, the mixture was extracted with dichloromethane $(3 \times 5 \mathrm{~mL})$ and washed with water $(3 \times 3 \mathrm{~mL})$. The combined organic layer was dried over anhydrous magnesium sulfate and concentrated in vacuo. The residue was purified with preparative TLC. $R_{f}=0.63$ (silica gel, petroleum ether/ethyl acetate $=$ $1 / 5$ as eluent). The pure 12a was obtained as a white solid. Yield $70 \%(33 \mathrm{mg}), \mathrm{m} . \mathrm{p} .142 .2-144.6^{\circ} \mathrm{C}$. ${ }^{31} \mathrm{P}-\mathrm{NMR}\left(162 \mathrm{MHz}, \mathrm{CDCl}_{3}\right) \delta=34.53$ (s). ${ }^{1} \mathrm{H}-\mathrm{NMR}\left(400 \mathrm{MHz}, \mathrm{CDCl}_{3}\right) \delta=7.73-7.62(\mathrm{~m}, 4 \mathrm{H}), 7.54-7.47$ 
$(\mathrm{m}, 2 \mathrm{H}), 7.44(\mathrm{dd}, J=6.9,3.3 \mathrm{~Hz}, 4 \mathrm{H}), 7.29(\mathrm{dd}, J=8.1,4.8 \mathrm{~Hz}, 4 \mathrm{H}), 7.25-7.19(\mathrm{~m}, 1 \mathrm{H}), 4.81(\mathrm{~s}, 1 \mathrm{H})$, $4.19(\mathrm{~s}, 1 \mathrm{H}), 2.48-2.27(\mathrm{~m}, 2 \mathrm{H}), 2.16-1.93(\mathrm{~m}, 2 \mathrm{H}) .{ }^{13} \mathrm{C}-\mathrm{NMR}\left(101 \mathrm{MHz}, \mathrm{CDCl}_{3}\right) \delta=139.3(\mathrm{~s}), 128.1(\mathrm{~d}$, $J=18.6 \mathrm{~Hz}), 127.1(\mathrm{~s}), 126.1(\mathrm{dd}, J=9.4,4.4 \mathrm{~Hz}), 123.9(\mathrm{dd}, J=11.7,1.6 \mathrm{~Hz}), 123.6(\mathrm{~s}), 122.6(\mathrm{~s}), 121.0$ (s), $72.6(\mathrm{~s}), 72.3(\mathrm{~s}), 71.9(\mathrm{~s}), 68.8(\mathrm{~d}, J=10.0 \mathrm{~Hz}), 26.6(\mathrm{~s}), 21.5(\mathrm{~s}), 20.8(\mathrm{~s})$. HRMS (ESI ${ }^{+}$) Calcd. for $\mathrm{C}_{21} \mathrm{H}_{21} \mathrm{O}_{2} \mathrm{P}\left[\mathrm{M}+\mathrm{H}^{+}\right]$: 337.1357, Found: 337.1362.

Dimenthyl 3-hydroxy-3-phenylprop-1-en-1-ylphosphonate, 12b

Compound 12b was prepared according to a similar procedure to that for the preparation of 12a, which was purified with preparative TLC with $R_{f}=0.68$ (silica gel, petroleum ether / ethyl acetate $=$ $1 / 3$ as eluent), and $\mathbf{1 2 b}$ was obtained as a yellow oil. Yield 55\% (26 mg). ${ }^{31} \mathrm{P}-\mathrm{NMR}\left(162 \mathrm{MHz}, \mathrm{CDCl}_{3}\right)$ $\delta=30.88(\mathrm{~s}, 50 \%), 30.86(\mathrm{~s}, 50 \%) .{ }^{1} \mathrm{H}-\mathrm{NMR}\left(400 \mathrm{MHz}, \mathrm{CDCl}_{3}\right) \delta=7.76-7.48(\mathrm{~m}, 1 \mathrm{H}), 7.35(\mathrm{~d}, J=4.2 \mathrm{~Hz}$, $3 \mathrm{H}), 7.27(\mathrm{~s}, 1 \mathrm{H}), 4.80(\mathrm{~s}, 1 \mathrm{H}), 4.24-4.09(\mathrm{~m}, 2 \mathrm{H}), 3.04(\mathrm{~d}, J=12.4 \mathrm{~Hz}, 1 \mathrm{H}), 2.22(\mathrm{~s}, 2 \mathrm{H}), 2.17-2.00(\mathrm{~m}, 4 \mathrm{H})$, $1.83-1.70(\mathrm{~m}, 3 \mathrm{H}), 1.68-1.58(\mathrm{~m}, 7 \mathrm{H}), 1.44(\mathrm{~d}, J=7.6 \mathrm{~Hz}, 3 \mathrm{H}), 1.25(\mathrm{~s}, 6 \mathrm{H}), 1.17-1.06(\mathrm{~m}, 2 \mathrm{H}), 0.90(\mathrm{~d}$, $J=6.2 \mathrm{~Hz}, 9 \mathrm{H}), 0.81-0.77(\mathrm{~m}, 3 \mathrm{H}), 0.76-0.73(\mathrm{~m}, 1 \mathrm{H}) .{ }^{13} \mathrm{C}-\mathrm{NMR}\left(101 \mathrm{MHz}, \mathrm{CDCl}_{3}\right) \delta=144.0(\mathrm{~s}), 128.4$ (s), $127.5(\mathrm{~d}, J=2.2 \mathrm{~Hz}), 125.8(\mathrm{~s}), 125.7(\mathrm{~s}), 77.2(\mathrm{~d}, J=4.4 \mathrm{~Hz}), 73.9(\mathrm{dd}, J=13.2,6.1 \mathrm{~Hz}), 48.7-48.6$ (m), 48.6-48.5 (m), $43.7(\mathrm{~d}, J=1.8 \mathrm{~Hz}), 43.1(\mathrm{~d}, J=6.0 \mathrm{~Hz}), 41.3(\mathrm{~s}), 34.6(\mathrm{~d}, J=15.5 \mathrm{~Hz}), 34.1(\mathrm{~s}), 32.2$ $(\mathrm{dd}, J=5.0,2.4 \mathrm{~Hz}), 31.6(\mathrm{~d}, J=1.7 \mathrm{~Hz}), 31.5(\mathrm{~s}), 29.0(\mathrm{~s}), 26.9(\mathrm{~s}), 25.5(\mathrm{~d}, J=4.9 \mathrm{~Hz}), 25.3(\mathrm{~s}), 22.8(\mathrm{~d}$, $J=3.3 \mathrm{~Hz}), 22.6(\mathrm{~d}, J=3.8 \mathrm{~Hz}), 21.9(\mathrm{~d}, J=2.9 \mathrm{~Hz}), 21.0(\mathrm{~d}, J=1.7 \mathrm{~Hz}), 15.8(\mathrm{~d}, J=2.3 \mathrm{~Hz}), 15.6(\mathrm{~d}$, $J=2.8 \mathrm{~Hz}), 14.1(\mathrm{~s}), 11.4(\mathrm{~s})$. HRMS (ESI ${ }^{+}$) Calcd. for $\mathrm{C}_{29} \mathrm{H}_{49} \mathrm{O}_{4} \mathrm{P}\left[\mathrm{M}+\mathrm{H}^{+}\right]$: 493.3447, Found: 493.3447.

\subsubsection{Reaction of $\mathbf{1}$ with Secondary Amine to Form Functional Phosphorus Derivatives}

\section{Dimenthyl 2-benzoyl-4-cyanobutylphosphonate, 13a}

To a solution of $1 \mathbf{d}(50 \mathrm{mg}, 0.098 \mathrm{mmol})$, palladium acetate $(1.1 \mathrm{mg}, 0.005 \mathrm{mmol}, 5 \mathrm{~mol} \%)$, and triphenyl phosphine $(5.1 \mathrm{mg}, 0.020 \mathrm{mmol}, 20 \mathrm{~mol} \%)$ in toluene $(0.5 \mathrm{~mL})$, pyrrolidine $(18 \mu \mathrm{L}$, $0.216 \mathrm{mmol}$ ) was added. The mixture was heated at $120^{\circ} \mathrm{C}$ for $24 \mathrm{~h}$. The removal of toluene and excess pyrrolidine in vacuo afforded the crude enamine 6 , which was used in situ. The crude 6 was dissolved in $0.5 \mathrm{~mL}$ of dry DMF, acrylonitrile $(19 \mu \mathrm{L}, 0.294 \mathrm{mmol})$ was added, and then the mixture was heated at $120^{\circ} \mathrm{C}$ for $36 \mathrm{~h}$. After cooling to room temperature, water $(2 \mathrm{~mL})$ was added and the mixture was extracted with dichloromethane $(3 \times 5 \mathrm{~mL})$. The combined organic layer was washed with water, dried over anhydrous magnesium sulfate, and concentrated in vacuo to give the crude product as a brown oil, which was purified with preparative TLC with $R_{f}=0.34$ (silica gel, dichloromethane/ethyl acetate= 5:1), to give pure 13a as a yellow oil. Yield $56 \%(28 \mathrm{mg}) .{ }^{31} \mathrm{P}-\mathrm{NMR}\left(162 \mathrm{MHz}, \mathrm{CDCl}_{3}\right) \delta=25.98(\mathrm{~s}, 50 \%)$, $25.93(\mathrm{~s}, 50 \%) .{ }^{1} \mathrm{H}-\mathrm{NMR}\left(400 \mathrm{MHz}, \mathrm{CDCl}_{3}\right) \delta=8.02(\mathrm{t}, J=7.9 \mathrm{~Hz}, 2 \mathrm{H}), 7.59(\mathrm{dd}, J=7.3,5.4 \mathrm{~Hz}, 1 \mathrm{H}), 7.48$ $(\mathrm{t}, J=7.5 \mathrm{~Hz}, 2 \mathrm{H}), 4.20-4.09(\mathrm{~m}, 2 \mathrm{H}), 3.99(\mathrm{dd}, J=12.4,6.2 \mathrm{~Hz}, 1 \mathrm{H}), 2.44-2.09(\mathrm{~m}, 6 \mathrm{H}), 2.07-1.68(\mathrm{~m}$, $4 \mathrm{H}), 1.61(\mathrm{~d}, J=9.0 \mathrm{~Hz}, 4 \mathrm{H}), 1.38(\mathrm{~d}, J=3.1 \mathrm{~Hz}, 2 \mathrm{H}), 1.15(\mathrm{tdd}, J=22.6,20.4,11.6 \mathrm{~Hz}, 3 \mathrm{H}), 1.00-0.92(\mathrm{~m}$, $2 \mathrm{H}), 0.86(\mathrm{dt}, J=11.4,6.4 \mathrm{~Hz}, 11 \mathrm{H}), 0.79(\mathrm{~d}, J=7.0 \mathrm{~Hz}, 2 \mathrm{H}), 0.75(\mathrm{dd}, J=10.6,4.4 \mathrm{~Hz}, 7 \mathrm{H}) .{ }^{13} \mathrm{C}-\mathrm{NMR}$ $\left(101 \mathrm{MHz}, \mathrm{CDCl}_{3}\right) \delta=200.5(\mathrm{dd}, J=19.8,10.3 \mathrm{~Hz}), 135.8(\mathrm{~d}, J=5.8 \mathrm{~Hz}), 133.7(\mathrm{~d}, J=4.3 \mathrm{~Hz}), 128.8(\mathrm{dd}$, $J=22.1,5.6 \mathrm{~Hz}), 118.8(\mathrm{~s}), 78.1(\mathrm{~d}, J=7.4 \mathrm{~Hz}), 77.9-76.8(\mathrm{~m}), 76.7(\mathrm{~s}), 48.7-48.1(\mathrm{~m}), 43.6(\mathrm{~d}, J=4.7 \mathrm{~Hz})$, $42.9(\mathrm{~d}, J=13.5 \mathrm{~Hz}), 39.2(\mathrm{dd}, J=15.9,3.9 \mathrm{~Hz}), 34.0(\mathrm{~d}, J=3.3 \mathrm{~Hz}), 31.5(\mathrm{dd}, J=10.7,3.9 \mathrm{~Hz}), 30.6(\mathrm{~s})$, $29.1(\mathrm{~s}), 28.3(\mathrm{dd}, J=9.1,3.9 \mathrm{~Hz}), 25.7(\mathrm{~d}, J=9.8 \mathrm{~Hz}), 25.4(\mathrm{~d}, J=6.3 \mathrm{~Hz}), 22.7(\mathrm{t}, J=6.3 \mathrm{~Hz}), 21.9(\mathrm{~d}$, $J=3.0 \mathrm{~Hz}), 20.9(\mathrm{~d}, J=11.1 \mathrm{~Hz}), 15.9-15.4(\mathrm{~m}), 14.8(\mathrm{~d}, J=13.2 \mathrm{~Hz}) . \mathrm{IR}(\mathrm{KBr}) v / \mathrm{cm}^{-1}: 2247,1685,1181$, 755, 558. HRMS $\left(\mathrm{ESI}^{+}\right)$Calcd. for $\mathrm{C}_{32} \mathrm{H}_{50} \mathrm{NO}_{4} \mathrm{P}\left[\mathrm{M}+\mathrm{Na}^{+}\right]: 566.3375$, Found: 566.3373 .

\section{Ethyl 4-dimenthylphosphoryl methyl-5-oxo-5-phenylpentanoate, 13b}

The enamine 6 was prepared similarly to 13a, which was reacted with ethyl acrylate $(31 \mu \mathrm{L}$, $0.294 \mathrm{mmol}$ ) for $48 \mathrm{~h}$ at $120^{\circ} \mathrm{C}$. The crude product was purified with preparative TLC with $R_{f}=0.41$ (silica gel, dichloromethane/ ethyl acetate $=20: 1)$ to give pure $\mathbf{1 3 b}$ as a yellow oil. Yield $28 \%(15 \mathrm{mg})$. ${ }^{31} \mathrm{P}-\mathrm{NMR}\left(162 \mathrm{MHz}, \mathrm{CDCl}_{3}\right) \delta=27.98(\mathrm{~s}, 50 \%), 27.85(\mathrm{~s}, 50 \%) .{ }^{1} \mathrm{H}-\mathrm{NMR}\left(400 \mathrm{MHz}, \mathrm{CDCl}_{3}\right) \delta=8.02(\mathrm{t}$, $J=9.1 \mathrm{~Hz}, 2 \mathrm{H}), 7.55(\mathrm{~d}, J=6.3 \mathrm{~Hz}, 1 \mathrm{H}), 7.46(\mathrm{t}, J=7.1 \mathrm{~Hz}, 2 \mathrm{H}), 4.14-4.04(\mathrm{~m}, 4 \mathrm{H}), 3.96(\mathrm{~s}, 1 \mathrm{H}), 2.44-2.17$ $(\mathrm{m}, 4 \mathrm{H}), 2.11(\mathrm{~d}, J=7.1 \mathrm{~Hz}, 2 \mathrm{H}), 2.05-1.87(\mathrm{~m}, 3 \mathrm{H}), 1.82(\mathrm{~d}, J=17.5 \mathrm{~Hz}, 1 \mathrm{H}), 1.72(\mathrm{~s}, 1 \mathrm{H}), 1.59(\mathrm{~s}, 4 \mathrm{H})$, 
$1.36(\mathrm{~s}, 2 \mathrm{H}), 1.19(\mathrm{t}, J=7.1 \mathrm{~Hz}, 3 \mathrm{H}), 1.13-1.01(\mathrm{~m}, 2 \mathrm{H}), 0.98-0.89(\mathrm{~m}, 4 \mathrm{H}), 0.85(\mathrm{t}, J=6.3 \mathrm{~Hz}, 8 \mathrm{H}), 0.80(\mathrm{~d}$, $J=6.5 \mathrm{~Hz}, 3 \mathrm{H}), 0.77(\mathrm{~d}, J=7.5 \mathrm{~Hz}, 4 \mathrm{H}), 0.74-0.70(\mathrm{~m}, 4 \mathrm{H}) .{ }^{13} \mathrm{C}-\mathrm{NMR}\left(101 \mathrm{MHz}, \mathrm{CDCl}_{3}\right) \delta=201.5(\mathrm{dd}$, $J=9.3,6.7 \mathrm{~Hz}), 172.6(\mathrm{~s}), 136.6(\mathrm{~d}, J=5.3 \mathrm{~Hz}), 133.2(\mathrm{~d}, J=4.0 \mathrm{~Hz}), 128.6(\mathrm{dd}, J=7.5,4.2 \mathrm{~Hz}), 77.7(\mathrm{~d}$, $J=7.3 \mathrm{~Hz}), 77.1(\mathrm{~s}), 76.8(\mathrm{~s}), 60.4(\mathrm{~s}), 48.5(\mathrm{~d}, J=6.7 \mathrm{~Hz}), 48.4(\mathrm{~d}, J=3.7 \mathrm{~Hz}), 48.3(\mathrm{~d}, J=7.0 \mathrm{~Hz}), 43.5(\mathrm{~d}$, $J=8.3 \mathrm{~Hz}), 42.8(\mathrm{~d}, J=15.3 \mathrm{~Hz}), 39.5(\mathrm{~d}, J=3.9 \mathrm{~Hz}), 39.2(\mathrm{~s}), 34.0(\mathrm{t}, J=3.7 \mathrm{~Hz}), 31.5(\mathrm{~d}, J=3.5 \mathrm{~Hz}), 31.4$ $(\mathrm{s}), 31.3(\mathrm{~d}, J=3.0 \mathrm{~Hz}), 31.2(\mathrm{~s}), 30.2(\mathrm{~d}, J=9.7 \mathrm{~Hz}), 28.8(\mathrm{dd}, J=26.9,12.9 \mathrm{~Hz}), 25.6(\mathrm{~s}), 25.5(\mathrm{~s}), 25.2(\mathrm{~d}$, $J=3.0 \mathrm{~Hz}), 22.7(\mathrm{~d}, J=3.4 \mathrm{~Hz}), 22.6(\mathrm{~d}, J=4.6 \mathrm{~Hz}), 21.9(\mathrm{~s}), 21.0(\mathrm{~s}), 20.9(\mathrm{~s}), 15.7(\mathrm{~d}, J=5.0 \mathrm{~Hz}), 15.6(\mathrm{~s})$, 14.1 (s). HRMS (ESI ${ }^{+}$) Calcd. for $\mathrm{C}_{34} \mathrm{H}_{55} \mathrm{NO}_{6} \mathrm{P}\left[\mathrm{M}+\mathrm{Na}^{+}\right]$: 613.3634, Found: 613.3649.

\subsubsection{Physical Characterization of the Crystals}

Single-crystal X-ray diffraction data for two Schiff base copper complexes, complex 1 and complex 2, were conducted on a Bruker-AXS CCD diffractometer, which was equipped with graphite-monochromated Mo-Ka radiation $(\lambda=0.71073 \AA)$, at $298 \mathrm{~K}$. All absorption corrections were applied using a multi-scan technique. The structures were solved by the direct method and refined through the full-matrix least-squares method on $\mathrm{F}^{2}$ using the SHELXTL 97 crystallographic software package. The FT-IR spectra were recorded with $\mathrm{KBr}$ as pellets in the range $4000-400 \mathrm{~cm}^{-1}$ on a Nicolet 170 SXFT/IR spectrometer (Nicolet, Madison, WI, USA).

\section{Conclusions}

In summary, the reaction of the phosphorus-containing allyl chloride $\mathbf{1}$ with an amine could form enamine or imino compounds 6 or 7 in the presence of a palladium catalyst. The reaction was confirmed to proceed via vinyl chloride as an intermediate that was formed from the isomerization of 1. Different to the reported coupling between aryl halides and an amine, the current reaction occurred in the presence of an amine and triphenyl phosphine. The imine or enamine could be converted to derivatives containing various functional groups, such as $\gamma$-keto, amino, and hydroxyl groups. When secondary amines were used, the enamine could be alkylated at the $\beta$-position via a Stork reaction. Utilizing the current procedure, a wide variety of phosphorus compounds having functional groups were obtained, which we hope will have important uses in biochemistry, pharmacology, and organic synthesis.

Supplementary Materials: The following are available online at http:/ /www.mdpi.com/2073-4344/8/5/194/s1, Part 1. Crystallographic Information. Part 2. The NMR spectrum for the mechanism of $1 \mathrm{f}$ with benzyl amine. Part 3. Selected ${ }^{31} \mathrm{P},{ }^{1} \mathrm{H}$ and ${ }^{13} \mathrm{C}$-NMR spectroscopy of $1,2,4,5,11,12$ and 13.

Author Contributions: J.-H.W. conducted most of the experimental operations and analysis. Q.L. conducted the analysis of NMR spectrum. J.-J.Y. prepared several compounds. S.-Z.N. analyzed the identification of crystal structures. Q.X. conducted the high resolution mass spectrometry of the compounds. C.-Q.Z. edited the manuscript and polished the language of the manuscript.

Acknowledgments: The authors acknowledge the financial support of the Natural Science Foundation of China (grant No. 20772055) and the Natural Science Foundation of Shandong Province (grant Nos. ZR2016BM18 and ZR2014BP007).

Conflicts of Interest: The authors declare no conflict interest.

\section{References and Notes}

1. Lejczak, B.; Kafarski, P.; Zygmunt, J. Inhibition of aminopeptidases by aminophosphonates. Biochemistry 1989, 28, 3549-3555. [CrossRef] [PubMed]

2. Oleksyszyn, J.; Powers, J.C. Amino acid and peptide phosphonate derivatives as specific inhibitors of serine peptidases. Methods Enzymol. 1994, 244, 423-441. [CrossRef] [PubMed]

3. Quin, L.D. A Guide to Organophosphorus Chemistry; John Wiley \& Sons: New York, NY, USA, 2000; ISBN 9780471318248.

4. Kafarski, P.; Lejczak, B. Aminophosphonic Acids of Potential Medical Importance. Curr. Med. Chem. Anti Cancer Agents 2001, 1, 301-312. [CrossRef] [PubMed] 
5. Orsini, F.; Sello, G.; Sisti, M. Aminophosphonic acids and derivatives. Synthesis and biological applications. Curr. Med. Chem. 2010, 17, 264-289. [CrossRef] [PubMed]

6. Turcheniuk, K.V.; Kukhar, V.P.; Röschenthaler, G.-V.; Aceña, J.L.; Soloshonok, V.A.; Sorochinsky, A.E. Recent advances in the synthesis of fluorinated aminophosphonates and aminophosphonic acids. RSC Adv. 2013, 3, 6693-6716. [CrossRef]

7. Roberts, P.J.; Foster, G.A.; Sharif, N.A.; Collins, J.F. Phosphonate analogues of acidic amino acids: Inhibition of excitatory amino acid transmitter binding to cerebellar membranes and of the stimulation of cerebellar cyclic GMP levels. Brain Res. 1982, 238, 475-479. [CrossRef]

8. Kafarski, P.; Lejczak, B.; Mastalerz, P.; Dus, D.; Radzikowski, C. N-(Phosphonoacetyl)amino phosphonates. Phosphonate analogs of N-(phosphonoacetyl)-L-aspartic acid (PALA). J. Med. Chem. 1985, 28, 1555-1558. [CrossRef] [PubMed]

9. Kudzin, Z.H.; Kudzin, M.H.; Drabowicz, J.; Stevens, C.V. Aminophosphonic acids-phosphorus analogues of natural amino acids. Part 1: Syntheses of $\alpha$-aminophosphonic acids. Curr. Org. Chem. 2011, 15, 2015-2071. [CrossRef]

10. Foss, F.W., Jr.; Snyder, A.H.; Davis, M.D.; Rouse, M.; Okusa, M.D.; Lynch, K.R.; Macdonald, T.L. Synthesis and biological evaluation of gamma-aminophosdphonates as potent, subtype-selective sphingosine 1-phosphate receptor agonists and antagonists. Bioorg. Med. Chem. 2007, 15, 663-677. [CrossRef] [PubMed]

11. Jan, L.; Johan, R.; Marc, B.; Charles, T.; Rao, M. EP 0242246, 1987. Chem. Abstr. 1988, 109, 68192.

12. Li, Y.M.; Du, X.H.; Zhou, Q.H.; Chen, S.D. A novel procedure for the synthesis of ammonium glufosinate. Org. Prep. Proced. Int. 2014, 46, 565-568. [CrossRef]

13. Selvam, C.; Oueslati, N.; Lemasson, I.A.; Brabet, I.; Rigault, D.; Courtiol, T.; Cesarini, S.; Triballeau, N.; Bertrand, H.O.; Goudet, C.; et al. A virtual screening hit reveals new possibilities for developing group III metabotropic glutamate receptor agonists. J. Med. Chem. 2010, 53, 2797-2813. [CrossRef] [PubMed]

14. Zeiss, H.J. Enantioselective synthesis of both enantiomers of phosphinothricin via asymmetric hydrogenation of alpha-acylamido acrylates. J. Org. Chem. 1991, 56, 1783-1788. [CrossRef]

15. Ashburn, B.O.; Carter, R.G.; Zakharov, L.N. Synthesis of tetra-ortho-substituted, phosphorus-containing and carbonyl-containing biaryls utilizing a diels-alder approach. J. Am. Chem. Soc. 2007, 129, 9109-9116. [CrossRef] [PubMed]

16. Heller, B.; Gutnov, A.; Fischer, C.; Drexler, H.J.; Spannenberg, A.; Redkin, D.; Sundermann, C.; Sundermann, B. Phosphorus-bearing axially chiral biaryls by catalytic asymmetric cross-cyclotrimerization and a first application in asymmetric hydrosilylation. Chem. Eur. J. 2007, 13, 1117-1128. [CrossRef] [PubMed]

17. Nishida, G.; Noguchi, K.; Hirano, M.; Tanaka, K. Asymmetric assembly of aromatic rings to produce tetra-ortho-substituted axially chiral biaryl phosphorus compounds. Angew. Chem. Int. Ed. 2007, 46, 3951-3954. [CrossRef] [PubMed]

18. Ashburn, B.O.; Carter, R.G. Diels-Alder approach to polysubstituted biaryls: Rapid entry to tri- and tetra-ortho-substituted phosphorus-containing biaryls. Angew. Chem. Int. Ed. 2006, 45, 6737-6741. [CrossRef] [PubMed]

19. Doherty, S.; Knight, J.G.; Smyth, C.H.; Jorgenson, G.A. Electron-rich, bicyclic biaryl-like KITPHOS monophosphines via [4+2] cycloaddition between 1-alkynylphosphine oxides and anthracene: Highly efficient ligands for palladium-catalysed C-N and C-C Bond formation. Adv. Synth. Catal. 2008, 350, 1801-1806. [CrossRef]

20. Vicario, J.; Aparicio, D.; Palacios, F. Conjugate addition of amines to an $\alpha, \beta$-unsaturated imine derived from $\alpha$-aminophosphonate. Synthesis of $\lambda$-Amino- $\alpha$-dehydroaminophosphonates. J. Org. Chem. 2009, 74, 452-455. [CrossRef] [PubMed]

21. Cytlak, T.; Saweliew, M.; Kubicki, M.; Koroniak, H. Synthesis of trifluoromethyl $\gamma$-aminophosphonates by nucleophilic aziridine ring opening. Org. Biomol. Chem. 2015, 13, 10050-10059. [CrossRef] [PubMed]

22. Kaźmierczak, M.; Kubicki, M.; Koroniak, H. Preparation and characterization of $\alpha$-fluorinated- $\lambda$-aminophosphonates. J. Fluor. Chem. 2014, 167, 128-134. [CrossRef]

23. Sambiagio, C.; Marsden, S.P.; Blacker, A.J.; McGowan, P.C. Copper catalyzed Ullmann type chemistry: From mechanistic aspects to modern development. Chem. Soc. Rev. 2014, 43, 3525-3550. [CrossRef] [PubMed]

24. Surry, D.S.; Buchwald, S.L. Diamine ligands in copper-catalyzed reactions. Chem. Sci. 2010, 1, 13-31. [CrossRef] [PubMed] 
25. Monnier, F.; Taillefer, M. Catalytic C-C, C-N and C-O Ullmann-Type coupling reactions. Angew. Chem. Int. Ed. 2009, 48, 6954-6971. [CrossRef] [PubMed]

26. Rivero, M.R.; Buchwald, S.L. Copper-catalyzed vinylation of hydrazides. A regioselective entry to highly substituted pyrroles. Org. Lett. 2007, 9, 973-976. [CrossRef] [PubMed]

27. Kong, L.K.; Zhou, Y.Y.; Huang, H.; Yang, Y.; Liu, Y.Y.; Li, Y.Z. Copper-catalyzed synthesis of substituted quinolines via C-N coupling/condensation from ortho-acylanilines and alkenyl iodides. J. Org. Chem. 2015, 80, 1275-1278. [CrossRef] [PubMed]

28. Li, E.; Xu, X.B.; Li, H.F.; Zhang, H.M.; Xu, X.L.; Yuan, X.Y.; Li, Y.Z. Copper-catalyzed synthesis of five-membered heterocycles via double $\mathrm{C}-\mathrm{N}$ bond formation: An efficient synthesis of pyrroles, dihydropyrroles, and carbazoles. Tetrahedron 2009, 65, 8961-8968. [CrossRef]

29. Bao, W.L.; Liu, Y.Y.; Lv, X. Mild Copper(I) Iodide/ $\beta$-keto ester catalyzed coupling reactions of styryl bromides with phenols, thiophenols, and imidazoles. Synthesis 2008, 12, 1911-1917. [CrossRef]

30. Martín, R.; Cuenca, A.; Buchwald, S.L. Sequential copper-catalyzed vinylation/cyclization: An efficient synthesis of functionalized oxazoles. Org. Lett. 2007, 9, 5521-5524. [CrossRef] [PubMed]

31. He, G.; Wang, J.; Ma, D.W. Highly convergent route to cyclopeptide alkaloids. Total synthesis of ziziphine N. Org. Lett. 2007, 9, 1367-1369. [CrossRef] [PubMed]

32. Yuan, X.Y.; Xu, X.B.; Zhou, X.B.; Yuan, J.W.; Mai, L.G.; Li, Y.Z. Copper-catalyzed double N-alkenylation of amides: An efficient synthesis of di- or trisubstituted N-acylpyrroles. J. Org. Chem. 2007, 72, 1510-1513. [CrossRef] [PubMed]

33. Martín, R.; Rivero, M.R.; Buchwald, S.L. Domino Cu-catalyzed C-N coupling/hydroamidation: A highly efficient synthesis of nitrogen heterocycles. Angew. Chem. Int. Ed. 2006, 45, 7079-7082. [CrossRef] [PubMed]

34. Trost, B.M.; Stiles, D.T. Synthesis of allenamides by Copper-catalyzed coupling of allenyl halides with amides, carbamates, and ureas. Org. Lett. 2005, 7, 2117-2120. [CrossRef] [PubMed]

35. Hu, T.S.; Li, C.Z. Synthesis of lactams via Copper-catalyzed intramolecular vinylation of amides. Org. Lett. 2005, 7, 2035-2038. [CrossRef] [PubMed]

36. Pan, X.H.; Cai, Q.; Ma, D.W. CuI/N, N-dimethylglycine-catalyzed coupling of vinyl halides with amides or carbamates. Org. Lett. 2004, 6, 1809-1812. [CrossRef] [PubMed]

37. Coleman, R.S.; Liu, P.H. Divergent and stereocontrolled synthesis of the enamide side chains of oximidines I/II/III, salicylihalamides A/B, lobatamides A/D, and CJ-12,950. Org. Lett. 2004, 6, 577-580. [CrossRef] [PubMed]

38. Han, C.; Shen, R.C.; Su, S.; Porco, J.A., Jr. Copper-Mediated Synthesis of N-acyl vinylogous carbamic acids and derivatives: Synthesis of the antibiotic CJ-15,801. Org. Lett. 2004, 6, 27-30. [CrossRef] [PubMed]

39. Son, S.; Fu, G.C. Nickel-catalyzed asymmetric Negishi cross-couplings of secondary allylic chlorides with alkylzincs. J. Am. Chem. Soc. 2008, 130, 2756-2757. [CrossRef] [PubMed]

40. Stork, G.; Brizzolarha, A.; Landesman, H.; Szmuszkovicz, J.; Terrell, R. The enamine alkylation and acylation of carbonyl compounds. J. Am. Chem. Soc. 1963, 85, 207-222. [CrossRef]

41. Ji, S.Y.; Sun, Y.M.; Zhang, H.; Hou, Q.G.; Zhao, C.Q. Phosphonium salt induced stereoselective allylic rearrangement during chlorination of $\alpha$-hydroxyallylphosphinates. Tetrahedron Lett. 2014, 55, 5742-5744. [CrossRef]

42. The mechanism for the copper-promoted formation of $4 \mathbf{d}^{\prime}$ was not studied in detail. On ${ }^{1} \mathrm{H}-\mathrm{NMR}$ and gCOSY spectrum, the peak of $\alpha$-proton located at 7.41, which was overlapped by the signals of phenyl.

43. On ${ }^{1} \mathrm{H}-\mathrm{NMR}$ spectrum, 1a gave two groups of peaks at 6.98 and $6.19 \mathrm{ppm}$ that were assigned as $\alpha$ - and $\beta$-vinyl protons, respectively. In contrast, $4 \mathbf{a}$ only gave one kind of vinyl proton.

44. Shan, C.; Chen, F.; Pan, J.; Gao, Y.; Xu, P.; Zhao, Y. Zn(OTf $)_{2}$-catalyzed phosphinylation of propargylic alcohols: Access to $\gamma$-ketophosphine oxides. J. Org. Chem. 2017, 82, 11659-11666. [CrossRef] [PubMed]

45. Wang, J.P.; Nie, S.Z.; Zhou, Z.Y.; Ye, J.J.; Wen, J.H.; Zhao, C.Q. Preparation of optically pure tertiary phosphine oxides via the addition of $P$-stereogenic secondary phosphine oxide to activated alkenes. J. Org. Chem. 2016, 81, 7644-7653. [CrossRef] [PubMed] 
46. A pair of stereoisomers, deriving from $\alpha$ - and $\gamma$-chiral carbon atoms, respectively, was observed for 11gb and $11 \mathbf{g b}^{\prime}$. On ${ }^{31} \mathrm{P}-\mathrm{NMR}$ spectrum, the two pairs of single peaks were observed at $45.80 / 45.34(\Delta \delta=0.46)$ and $43.44 / 43.28 \mathrm{ppm}(\Delta \delta=0.16)$, and the former was assigned as $\alpha$-stereoisomers $\mathbf{1 1 \mathbf { g b } ^ { \prime }}$, on the basis of the shorter distance thence the stronger interaction with chiral phosphorus.

47. Procedure of Stork reaction: The pyrrolidine enamine 6 was prepared from heating $1 \mathbf{d}$ and pyrrolidine $(2.2$ equiv.) with $\mathrm{Pd}(\mathrm{OAc})_{2}(5 \mathrm{~mol} \%)$, triphenyl phosphine $(20 \mathrm{~mol} \%)$ in toluene at $120^{\circ} \mathrm{C}$. After removal solvent, the residue was heated with ethyl acrylate or acrylonitrile ( 3 equiv.) at $120^{\circ} \mathrm{C}$.

2018 by the authors. Licensee MDPI, Basel, Switzerland. This article is an open access article distributed under the terms and conditions of the Creative Commons Attribution (CC BY) license (http:/ / creativecommons.org/licenses/by/4.0/). 